\title{
Aberrant chromatin landscape following loss of the H3.3 chaperone Daxx in haematopoietic precursors leads to Pu.1-mediated neutrophilia and inflammation
}

\author{
Julia P. Gerber1,2,11, Jenny Russ ${ }^{101,11}$, Vijay Chandrasekar (1) ', Nina Offermann', Hang-Mao Lee', \\ Sarah Spear ${ }^{3}$, Nicola Guzzi ${ }^{4}$, Simona Maida', Sundararaghavan Pattabiraman', Ruoyu Zhang1, \\ Amir H. Kayvanjoo5, Preeta Datta², Jagath Kasturiarachchi², Teresa Sposito', Natalia Izotova², \\ Kristian Händler ${ }^{6}$, Peter D. Adams $\mathbb{1}^{7}$, Teresa Marafioti ${ }^{2,8}$, Tariq Enver ${ }^{(2}{ }^{2}$, Jörg Wenzel ${ }^{9}$, \\ Marc Beyer ${ }^{1,6}$, Elvira Mass ${ }^{5}$, Cristian Bellodi ${ }^{4}$, Joachim L. Schultze ${ }^{1}{ }^{1,6,0}$, Melania Capasso $(1)$, \\ Rachael Nimmo ${ }^{2}$ and Paolo Salomoni ${ }^{1,2}$ 叫
}

\begin{abstract}
Defective silencing of retrotransposable elements has been linked to inflammageing, cancer and autoimmune diseases. However, the underlying mechanisms are only partially understood. Here we implicate the histone H3.3 chaperone Daxx, a retrotransposable element repressor inactivated in myeloid leukaemia and other neoplasms, in protection from inflammatory disease. Loss of Daxx alters the chromatin landscape, H3.3 distribution and histone marks of haematopoietic progenitors, leading to engagement of a Pu.1-dependent transcriptional programme for myelopoiesis at the expense of B-cell differentiation. This causes neutrophilia and inflammation, predisposing mice to develop an autoinflammatory skin disease. While these molecular and phenotypic perturbations are in part reverted in animals lacking both Pu.1 and Daxx, haematopoietic progenitors in these mice show unique chromatin and transcriptome alterations, suggesting an interaction between these two pathways. Overall, our findings implicate retrotransposable element silencing in haematopoiesis and suggest a cross-talk between the H3.3 loading machinery and the pioneer transcription factor Pu.1.
\end{abstract}

R epeat elements, including endogenous retroviral elements (ERVs), retrotransposable elements (RTEs) and telomeric repeats, represent more than half of the human genome. ERVs and RTEs (ERVs/RTEs) have emerged as important regulators of key DNA- and RNA-based cellular functions, such as assembly of transcriptional networks, splicing and mutagenesis ${ }^{1-3}$. Aberrant opening and/or expression of ERVs/RTEs has been linked to inflammageing, cancer and autoimmunity ${ }^{4-8}$. Given the pleiotropic roles of ERVs/RTEs and their potentially detrimental effects on cell and tissue homeostasis, several mechanisms are in place to regulate their accessibility and expression. In embryonic stem cells, DNA methylation and incorporation of the histone 3.3 (H3.3) variant via the Death domain-associated protein (Daxx)-Alpha-thalassaemia $\mathrm{X}$-linked mental retardation (Atrx) complex restricts accessibility to selected RTEs, such as intracisternal particles (IAPs) ${ }^{9,10}$. Loss of ERV/RTE silencing may activate their enhancer function in embryonic stem cells ${ }^{11}$. Notably, loss-of-function mutations in DNA methyltransferases, DAXX and ATRX are found in human cancer, including myeloid malignancies ${ }^{12-16}$. However, their impact on tissue homeostasis, inflammation and oncogenesis remains only partially understood.

Daxx regulates the HSC chromatin landscape and transcriptome

Expression of Daxx, Atrx and the other $\mathrm{H} 3.3$ chaperone Hira ${ }^{17}$ during haematopoiesis is enriched in long-term haematopoietic stem cells (LT-HSCs), megakaryocyte-erythrocyte progenitors and B cells (Extended Data Fig. 1a; based on ${ }^{18}$ ). To assess the impact of Daxx loss on the chromatin landscape of haematopoietic stem and progenitor cells (HSPCs), we crossed a conditional Daxx-knockout (KO) line with Rosa26CreERT2 mice (Extended Data Fig. 1b). Sorted LT-HSCs, common myeloid progenitors (CMPs) and granulocyte-monocyte progenitors (GMPs) from wild-type (WT; $\left.\operatorname{Daxx}^{+/+} ; \mathrm{RosaCre}^{\mathrm{ERT} 2+/-}\right)$ and Daxx-KO $\left(\operatorname{Daxx}^{\mathrm{F} / \mathrm{F}} ; \mathrm{RosaCre}^{\mathrm{ERT2+l}}\right)$ mice were processed 3 weeks post tamoxifen treatment/induction (w.p.i.) for transposase-accessible chromatin sequencing (ATAC-seq; Fig. 1a). Principal component analysis (PCA) showed separate clustering of WT and Daxx-KO samples (Extended Data Fig. 1c).

1German Center for Neurodegenerative Diseases (DZNE), Bonn, Germany. 2Department of Cancer Biology, UCL Cancer Institute, London, UK. ${ }^{3}$ Barts Cancer Institute, Queen Mary University of London, London, UK. ${ }^{4}$ Division of Molecular Hematology, Department of Laboratory Medicine, Lund Stem Cell Center, Faculty of Medicine, Lund University, Lund, Sweden. ${ }^{5}$ Life and Medical Sciences (LIMES) Institute, Developmental Biology of the Immune System, University of Bonn, Bonn, Germany. ${ }^{6}$ Platform for Single Cell Genomics and Epigenomics (PRECISE) at the German Center for Neurodegenerative Diseases

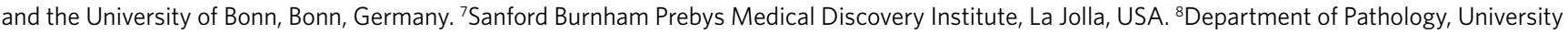
College London, London, UK. ${ }^{9}$ Department of Dermatology and Allergy, University Medical Center, Bonn, Germany. ${ }^{10} \mathrm{Genomics}$ and Immunoregulation, LIMES Institute, University of Bonn, Bonn, Germany. ${ }^{11 T h e s e ~ a u t h o r s ~ c o n t r i b u t e d ~ e q u a l l y: ~ J u l i a ~ P . ~ G e r b e r, ~ J e n n y ~ R u s s . ~} \bowtie e-m a i l:$ Paolo.Salomoni@dzne.de 
While the number of distal regions opening or closing in CMPs or GMPs compared with LT-HSCs were similar in the WT mice, substantially fewer distal regions were open in the Daxx-KO animals (Fig. 1b); most remained closed, suggesting Daxx-KO CMPs and GMPs became more restricted in their gene expression.

By overlapping accessible regions with known haematopoietic enhancers, we found more enhancers opening in KO LT-HSCs $(2,509)$ compared with CMPs and GMPs $(1,040$ and 716 , respectively; Fig. 1c). Based on unsupervised clustering (Fig. 1d), one set of enhancers showed increased read counts in KO LT-HSCs compared with WT LT-HSCs but reduced counts in CMPs and GMPs. Another set displayed similar counts in WT CMPs and GMPs as well as KO LT-HSCs, suggesting that chromatin features of Daxx-KO LT-HSCs may resemble myeloid-committed progenitors. The KO LT-HSCs displayed increased general opening of enhancers, including those overlapping with ERVs, in accordance with a Daxx-repressive role (Fig. 1e). ERV-overlapping enhancers were enriched in transcription factor motifs for the master regulators of haematopoiesis Pu.1 and Ets1 (Fig. 1f). Neighbouring genes displayed enrichment in immune-cell function and pathways associated with myeloid-committed progenitors (Extended Data Fig. 1d,e).

More acute effects of Daxx loss were assessed by collecting mice at $3 \mathrm{~d}$ post induction (d.p.i.). Like at 3 w.p.i., most changes occurred at distal regions. However, more sites displayed decreased $(8,421)$ than increased accessibility $(3,247)$. Despite a similar number of known haematopoietic enhancers being opened or closed $(1,020$ versus 1,022), more enhancers overlapping with ERVs opened (818 versus 617), with more pronounced differences at opened ERV-overlapping enhancers (Fig. 1g). RNA sequencing (RNA-seq; Extended Data Fig. If and Supplementary Table 1) showed increased expression of genes close to opened enhancers and ERV-overlapping enhancers (Fig. 1h,i). However, genes proximal to enhancers with reduced accessibility also showed increased expression, an effect potentially due to enhancer occupancy by transcription factors (Fig. 1i).

Ingenuity pathway analysis (IPA) and Gene Ontology suggested engagement of the double-stranded RNA (dsRNA)-recognition machinery and activation of interferon (IFN)-stimulated genes (ISGs; Fig. 1j-m and Supplementary Table 2). Key components of these pathways were indeed upregulated (for example, Irf3, Irf7, Mavs and $D d x 58$; Fig. 11,m) along with selected ERV subtypes, including the Daxx targets ERV1 and IAPs, but not non-ERV RTEs (Fig. 1n and Extended Data Fig. 1g). Enriched cell cycle-related pathways, along with upregulation of the proliferation marker Mki67 and downregulation of the quiescence gene Egr1, pointed at increased cell-cycle entry (Extended Data Fig. 1h and Supplementary Tables 1,2). Notably, upregulated genes such as $M x 1$, the Ifit cluster, Irf7 and Mki67 showed increased accessibility at ERV-overlapping enhancers and at Pu.1-binding sites (based on ${ }^{19}$; Fig. 1o,p and Extended Data Fig. 1h), suggesting a role for Pu.1 in their regulation. Spi1 (coding for Pu.1) regulatory elements showed increased opening but its expression was unaltered (Extended Data Fig. 1h).

In agreement with the reported role for Atrx in the regulation of the telomeric repeat-containing RNA (TERRA) ${ }^{10,20-23}$, TERRA-binding sites (TERRA-BS) ${ }^{24}$ were more open across all chromosomes in Daxx-KO LT-HSCs, including those at Mid1 and Erdr1, a potential HSC regulator and repressor of inflammatory skin disease ${ }^{25-27}$ (Extended Data Fig. 1i,j). Chromatin opening correlated with their downregulation, as was also observed at the autosomal Wls locus (Extended Data Fig. 1j). TERRA knockdown also reduced expression of these loci, whereas Atrx loss increased their expression ${ }^{23}$, suggesting that Daxx and Atrx may antagonize each other for TERRA regulation.

Overall, our data suggest that Daxx loss in LT-HSCs alters the chromatin landscape at ERV-overlapping enhancers and TERRA-BS, with potential implications for induction of an IFN type I response and cell-cycle entry.

\section{Daxx loss skews haematopoiesis towards myeloid differentiation}

We next investigated the effect of Daxx loss on haematopoiesis. The overall cell numbers in the KO bone marrow (BM) were significantly reduced at 3 d.p.i. (Fig. 2a), whereas the frequencies and numbers of LT-HSCs, c-Kit ${ }^{+}$Lineage (Lin)-Sca- $1^{+}$(KLS) cells and myeloid-restricted multipotent progenitor (MPP) 3 cells (Fig. 2b) were increased. Both GMPs and lymphoid-biased MPP4 cells showed increased frequencies. The neutrophil frequency in the $\mathrm{BM}$ was augmented, whereas the frequency and number of $\mathrm{B} 220^{+}$ cells were reduced (Fig. 2c). The B-cell defect encompassed pro-B, pre-B, and immature and mature B cells (Fig. 2d). The neutrophil frequency and number in the spleen were increased, whereas the monocyte number was reduced. B cells were unaffected and remained viable (Fig. 2e,f). At 2 w.p.i., the frequency and number of neutrophils were higher, whereas those of B cells were lower (Fig. 2g). Thus, Daxx loss causes early expansion of stem and progenitor cells, followed by increased BM and peripheral accumulation of neutrophils at the expense of B cells.

Fig. 1 | Daxx loss alters the chromatin landscape and transcription in LT-HSCs. a, Overview scheme of haematopoiesis. ST-HSC, short-term haematopoietic stem cells; MegE, megakaryocyte-erythrocyte; MEP, megakaryocyte-erythrocyte progenitor; GM, granulocyte-monocyte. b, Heatmap of the number of distal peaks that were open or closed in CMPs (open, 8,753 in Daxx-KO and 18,194 in Daxx-WT cells; closed, 28,724 in Daxx-KO and 18,465 in Daxx-WT cells) and GMPs (open, 12,713 in Daxx-KO and 22,833 in Daxx-WT cells; closed, 33,374 in Daxx-KO and 23,703 in Daxx-WT cells) compared with HSCs. c, Heatmap of enhancer-overlapping distal peaks that were open or closed in Daxx-KO compared with WT cells. b,c, >, open peaks; and <, closed peaks. d, Heatmap showing scaled read counts within the enhancer-overlapping distal peaks that were open in Daxx-KO LT-HSCs compared with WT cells. e, Total number of peaks of LT-HSCs that closed or opened in known enhancers following Daxx KO. f, Top-ten transcription factor motifs enriched in ERV-overlapping enhancer peaks open in Daxx-KO LT-HSCs. g, ATAC-seq coverage around the centre of enhancers overlapping ERVs. h, RNA-seq coverage across gene bodies (left) and around the TSS (right) of genes closest to opened enhancers overlapping ERVs. TES, transcription end site. i, Change in accessibility at enhancers overlapping ERVs versus changes in gene expression of the closest gene. Numbers show the total number of enhancer-gene pairs per quadrant. j, Summary plot of IPA analysis for the RNA-seq of LT-HSCs. Predicted activation of protein or biofunction is indicated in orange and predicted inhibition of the displayed protein or biofunction in blue. $\mathbf{k}$, Top-five activated and repressed IPA canonical pathways. Data are the activation $Z$-scores from IPA Fisher's exact tests with multiple testing-adjusted $P<0.05$. Activation $Z$-score $>2$, increased activation; activation $Z$-score $<-2$, increased inhibition. cyt., cytoplasmic. I, Heatmap showing expression of interferon-responsive genes and dsRNA-recognition machinery in LT-HSCs. m, Genome browser tracks of the Ddx58 (top) and Ifih1 (bottom) genes. Transcript structure and position are shown below. $\mathbf{n}$, Counts per million of repeat-element families in the LT-HSC RNA-seq data. Boxplots show the minimum and maximum values (box boundaries) and the mean (horizontal line). o, Genome browser track of the $M \times 1$ gene regulatory region. Transcripts within the region are shown below. $\mathbf{p}$, Genome browser tracks of the Irf7 (right) and Ifit (left) gene clusters. Transcripts within the clusters are shown below. HSC/LT-HSC, long-term haematopoietic stem cells. a-f, $n=2$ independent biological samples analysed at 3 w.p.i. g-p, $n=2\left(\right.$ Daxx $^{+/+}$and Daxx $\left.{ }^{+/ F}\right)$ and 3 (Daxx KO) independent biological samples collected at 3 d.p.i. Daxx F/F, Daxx KO and Daxx +/+, Daxx WT. Numerical source data are provided. 
To study the long-term effects of Daxx loss on haematopoiesis, we employed haematopoiesis-specific Cre lines. Daxx deletion during prenatal haematopoiesis using the Csf1rCre line failed to produce viable Daxx-KO pups (not shown), similar to a germline Daxx-KO line ${ }^{28}$. Haematopoiesis-specific and polyinosinic:polycytidylic acid (pI:pC)-inducible Daxx deletion in adult mice (using the Mx1Cre a

f

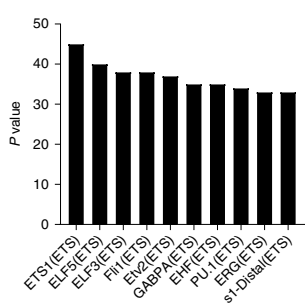

i

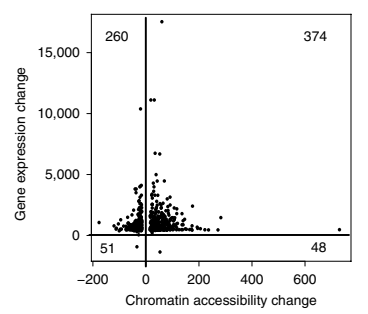

I

-
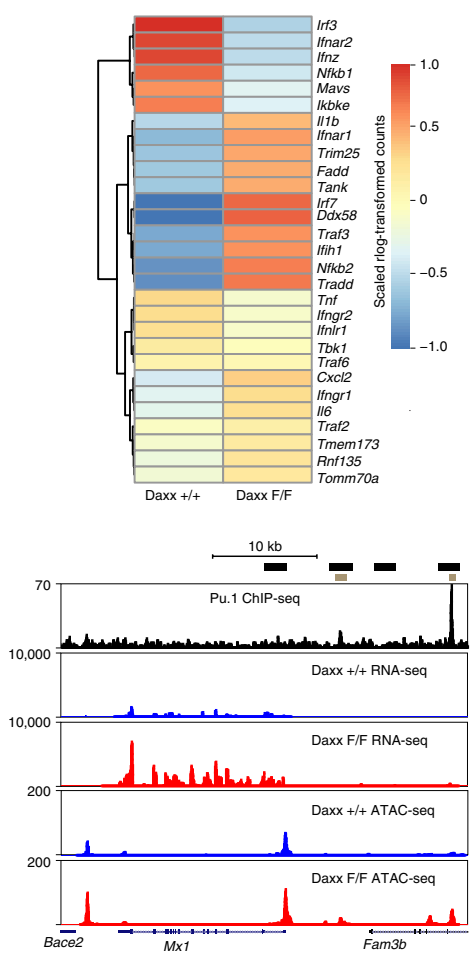

g

j b
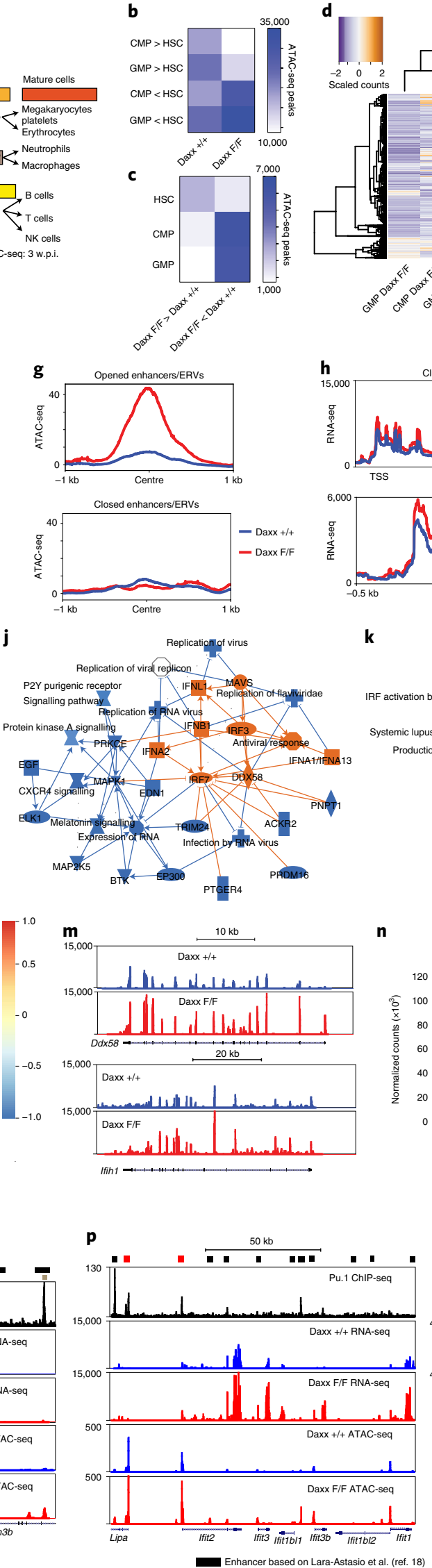
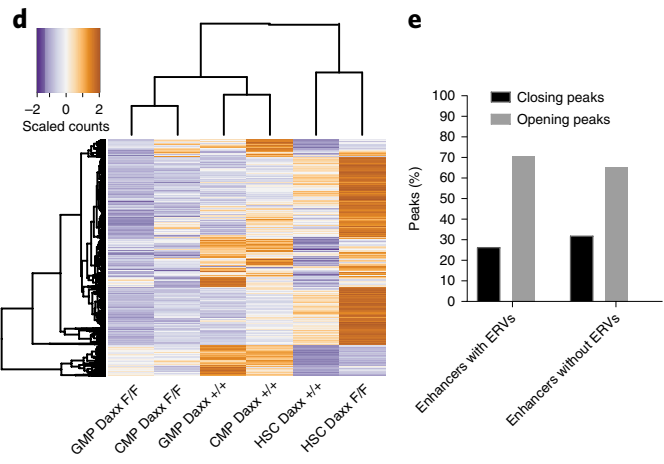
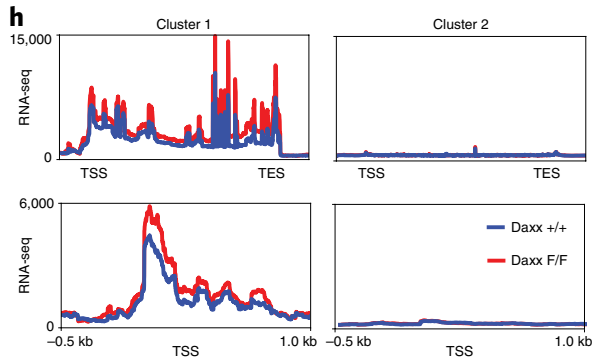

k
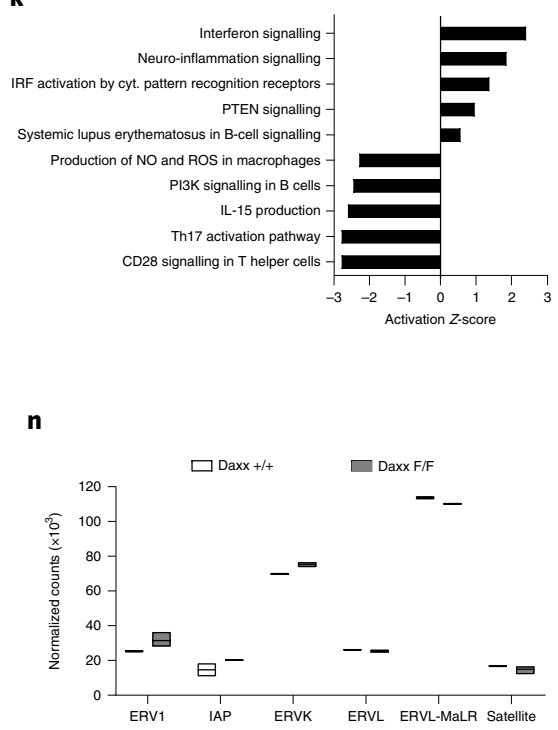
line ${ }^{29}$; Extended Data Fig. 2a-d) resulted in an increase in mature and immature neutrophils in the BM (Fig. 2h,i). Daxx-deficient mice displayed a trend towards lower BM cell counts but the number of white blood cells was higher in males after red-blood-cell (RBC) lysis (Extended Data Fig. 2e,f). These changes correlated with a higher frequency of $\mathrm{Ki} 67^{+}$cells in the BM as well as in HSPCs (Extended Data Fig. 2g). Similar to the $\mathrm{RosaCr} \mathrm{CRT}^{\mathrm{ERT}}$ model, the GMP frequency and number were increased (Fig. 2j,k). Although the frequency of common lymphoid progenitors (CLPs) was augmented, their total number was reduced (Fig. 2k,1); similar observations were made for LT-HSCs, and KLS, MPP3 and MPP4 cells (Extended Data Fig. 2h,i). Cytokines promoting myeloid differentiation, such as granulocyte-macrophage colony-stimulating factor (GM-CSF), interleukin (IL)-6, thrombopoietin and IL-34, were increased in plasma (Fig. $2 \mathrm{~m}$ ), correlating with higher frequencies of CD11 $\mathrm{b}^{+}$, Gr- $1^{\text {hi }}$ and Ly6G ${ }^{\text {hi }}$ myeloid cells (Extended Data Fig. 3a). While Ly6C ${ }^{\text {hi }}$ inflammatory monocytes were nearly absent (Extended Data Fig. 3a), neutrophils displayed increased number and frequency as well as diminished expression of the maturation marker Cxcr2 (Fig. 2n-p). The B-lymphocyte frequencies were reduced, with a marked drop from the pre pro-B cell stage onward (Extended Data Fig. 3b,c and Fig. 2q). The levels of Daxx protein were reduced in B cells with Daxx KO (Extended Data Fig. 3d), suggesting that a small number of recombined B cells are produced. Finally, the paler appearance of the bones of the Daxx-KO animals correlated with reduced levels of RBCs and erythroblast subpopulations, suggesting an impairment in BM erythropoiesis (Extended Data Fig. 3e,f).

Overall, Daxx loss markedly caused perturbations of haematopoietic differentiation compatible with a myeloid bias towards neutrophil production.

\section{Daxx-KO mice develop neutrophilia and inflammation}

Myeloid markers were increased in the peripheral blood (PB) of Daxx-KO mice, whereas the B-cell frequency decreased (Extended Data Fig. 4a). The levels of Cxcr2 were reduced in PB neutrophils, indicating a more immature status also outside of the BM (Extended Data Fig. 4b). A trend towards a reduction in the white-blood-cell count was observed (Extended Data Fig. 4c). Compared with controls, Daxx loss (Extended Data Fig. 4d-f) led to marked reduction in white pulp (Fig. 3a). The total number of spleen cells was unchanged, despite an increased frequency of myeloid cells (Fig. 3b,c). The CD41 ${ }^{+}$megakaryocyte frequency was higher (Extended Data Fig. 4a), although the number of platelets in the PB was only marginally increased (Extended Data Fig. 4c). Correlating with the reduction in white pulp, B cells were significantly reduced (Fig. 3c,g); the decrease affected follicular but not marginal B cells and to a minor degree plasma cells (Fig. $3 \mathrm{c}, \mathrm{h}$ ). Spleen macrophages $\left(\right.$ Ly6 $\left.6 \mathrm{C}^{\mathrm{lo} /-} \mathrm{Gr}-1^{10 /-}\right)$ were reduced, whereas monocytes were shifted towards a Ly6C $\mathrm{C}^{\text {intm }} \mathrm{Gr}-1^{\text {hi }}$ population (Fig. 3d and Extended Data Fig. 4g). We observed an increase in the neutrophil number and frequency, a higher eosinophil frequency, decreased Cxcr2 levels (Fig. 3e,f) and a trend towards an increased Ki67 $7^{+}$cell frequency (Extended Data Fig. 4h). Expansion of CD11b+ cells was observed but the $\mathrm{F} 4 / 80^{+}$monocytes/macrophages were unaltered (Fig. 3i-k). In contrast to $\mathrm{BM}$, erythroblast subpopulations were increased (Extended Data Fig. 4i), suggesting induction of extramedullary erythropoiesis, in accordance with normal RBC counts in the PB.

In agreement with reports linking neutrophilia and inflammation $^{30-33}$, we observed elevated levels of several key cytokines involved in myelopoiesis and inflammation (Fig. 4a), such as GM-CSF ${ }^{34}$ and IL-17A, the latter of which is linked to skin diseases $^{35,36}$. Interestingly, approximately $15 \%$ of the $\operatorname{Daxx}^{\mathrm{F} / \mathrm{F}} ; \mathrm{Mx} 1 \mathrm{Cre}^{+/-}$ mice developed skin lesions along with splenomegaly (Fig. 4b-e). These lesions resembled human pyoderma gangrenosum, a cutaneous neutrophilic autoinflammatory disease of unknown aetiology (Fig. 4 e) ${ }^{37}$ partly associated with myeloproliferative diseases. Similar to human pyoderma gangrenosum, the mouse lesions were characterized by thicker skin and sterile inflammation (Fig. 4e), with infiltration of neutrophils and neutrophil extracellular traps (Fig. 4f,g). The rather long latency of skin lesions correlated with a progressive increase in neutrophilia over time (Fig. 4h).

These findings suggest that progressive neutrophilia leads to systemic inflammation in Daxx-deficient mice.

\section{Limited role of Hira in adult haematopoiesis}

We next assessed the contribution of Histone cell-cycle regulator (Hira), which deposits H3.3 at euchromatin and facultative heterochromatin $^{38}$. In pI:pC-treated $\mathrm{Hira}^{\mathrm{F} / \mathrm{F}} ; \mathrm{M} \times 1 \mathrm{Cr}^{+/-}$mice (Extended Data Fig. $5 \mathrm{a}-\mathrm{c}$ ), no changes in erythroid marker frequencies or bone appearance were observed (Extended Data Fig. 5d,e). The frequencies of myeloid and lymphoid markers in the BM were significantly affected but markedly less than that observed in the Daxx-KO mice (Extended Data Fig. 5f,g). Although the frequency of neutrophils was significantly increased in the BM of the Hira-KO mice, it was unaffected in the spleen (Extended Data Fig. 5h,i). Unlike Daxx-deficient mice, the spleens of the Hira-KO mice were not enlarged (Extended Data Fig. 5j) and showed no or only minor changes in the myeloid, erythroid and lymphoid marker frequencies as well as organ structure (Extended Data Fig. 5k-m). No skin lesions were observed after extended monitoring. The marginal effects of Hira loss on haematopoiesis may be due to its lesser role and/or compensatory effects by Daxx given that the Daxx levels were higher in Hira-deficient cells (Extended Data Fig. 5c). The stronger phenotype described in a previous report based on Hira prenatal deletion ${ }^{39}$ could be caused by a more prominent Hira role during embryonic haematopoiesis.

Fig. 2 | Acute and chronic Daxx loss lead to perturbations of haematopoiesis. a, Total number of cells ( $n=5$ WT and 7 Daxx-KO mice). b, Frequency and total cell number of stem, multipotent and progenitor populations ( $n=4 \mathrm{WT}$ and $5 \mathrm{Daxx}-\mathrm{KO}$ mice). c, Frequency and total cell number of mature lymphoid and myeloid cells $(n=6$ WT and 7 Daxx-KO mice). d, Frequency and total cell numbers of B cell-progenitor populations $(n=4$ WT and 5 Daxx-KO mice). e, Frequency and total counts of mature lymphoid and myeloid cells ( $n=4$ mice per genotype). $\mathbf{f}$, Frequencies of apoptotic B cells ( $n=4$ WT and 5 Daxx-KO mice). $\mathbf{g}$, Frequency and total cell numbers of B cells and neutrophils at 2 w.p.i. ( $n=3$ mice per genotype). $\mathbf{h}$, Representative images of haematoxilin and eosin (H\&E) staining of bones ( $n=2$ independent experiments). Scale bars, $100 \mu \mathrm{m}$ (left) and $20 \mu \mathrm{m}$ (right; higher-magnification images). $\mathbf{i}$, Frequencies of immature and mature neutrophils ( $\mathrm{Gr} 1^{+} \mathrm{CD} 11 \mathrm{~b}^{+}$cells; $n=3$ mice per genotype). $\mathbf{j}$, Examples of the flow cytometry analysis of myeloid progenitor cells. $\mathbf{k}, \mathbf{l}$, Frequency $(\mathbf{k})$ and total count (I) of haematopoietic progenitors ( $n=3$ mice per genotype). $\mathbf{m}$, Concentration of different cytokines in plasma ( $n=6$ mice per genotype; analysis of variance and corresponding non-parametric Conover's test). TPO, thrombopoietin. $\mathbf{n}$, Frequencies of neutrophils ( $n=6$ mice per genotype; non-parametric Mann-Whitney test). o, Number of neutrophils ( $n=5$ mice per genotype; non-parametric Mann-Whitney test). p, Cxcr2 levels measured in mature neutrophils ( $n=3$ mice per genotype). MFU, mean fluorescent units. $\mathbf{q}$, Examples of flow cytometry analysis of $B$ cell progenitors and frequencies of $B$ cells and progenitors (right; $n=6$ mice per genotype; non-parametric Mann-Whitney test). a-g, RosaCreERT2 mice. h-q, Mx1Cre mice. $\mathbf{a}-\mathbf{d}, \mathbf{i}-\mathbf{I}, \mathbf{n}-\mathbf{q}$, BM. e-g, Spleen. $\mathbf{a}-\mathbf{g}, \mathbf{i}, \mathbf{k}, \mathbf{l}, \mathbf{p}$, Student's $t$-test. b-g,i,k-o, Boxplots show the minimum and maximum values (box boundaries) and the mean (horizontal line). a,p, Data are the mean \pm s.d. ${ }^{\star} P<0.05,{ }^{\star \star} P<0.01,{ }^{\star \star \star} P<0.001,{ }^{\star \star \star \star} P<0.0001$ and NS, not significant. Daxx F/F, Daxx KO and Daxx $+/+$, Daxx WT. Exact $P$ values and numerical source data are provided. 


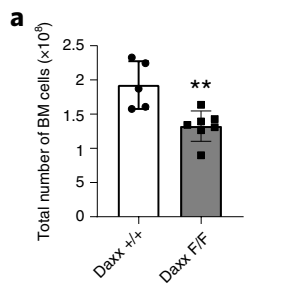

C
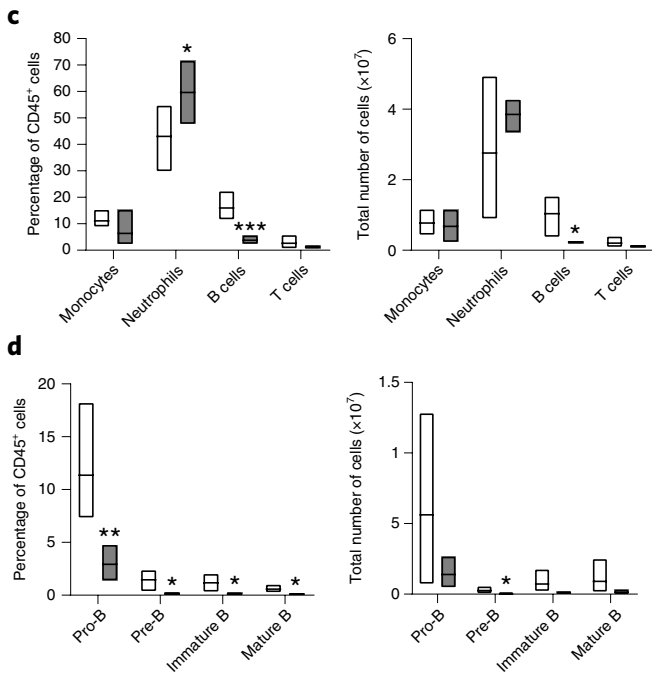

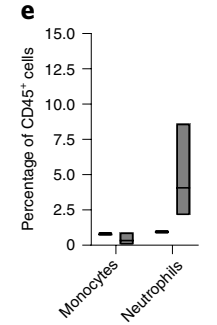

e

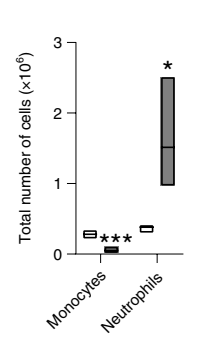

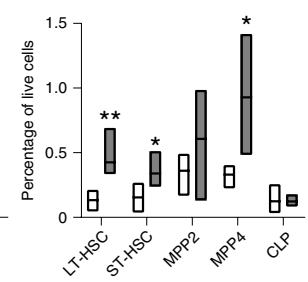

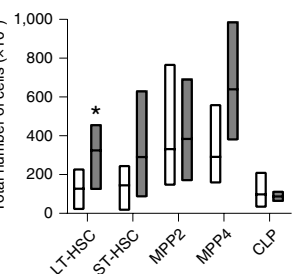

h

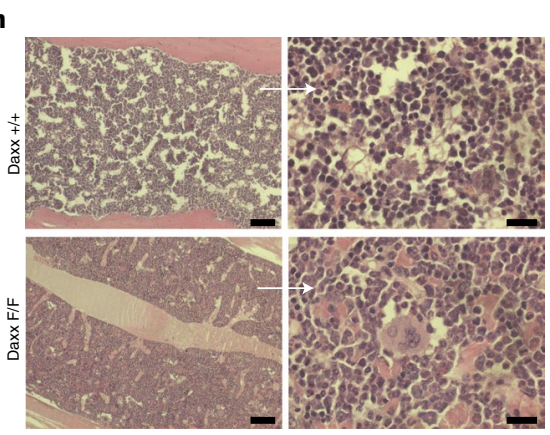

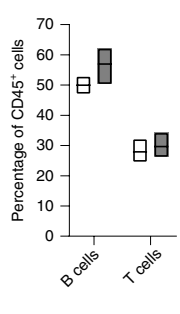

\section{,}

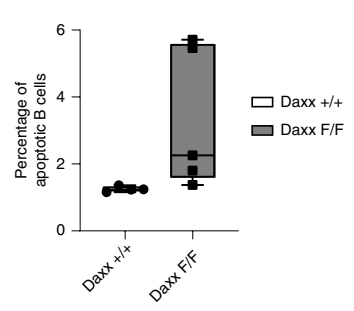

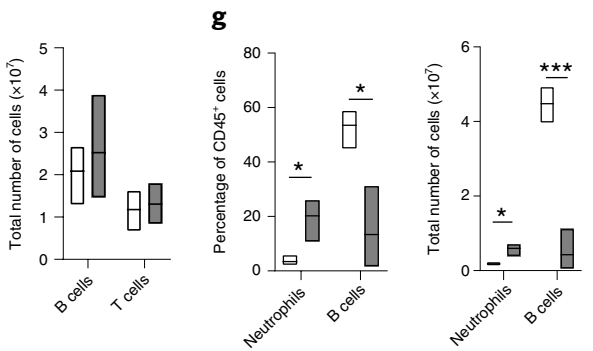

j
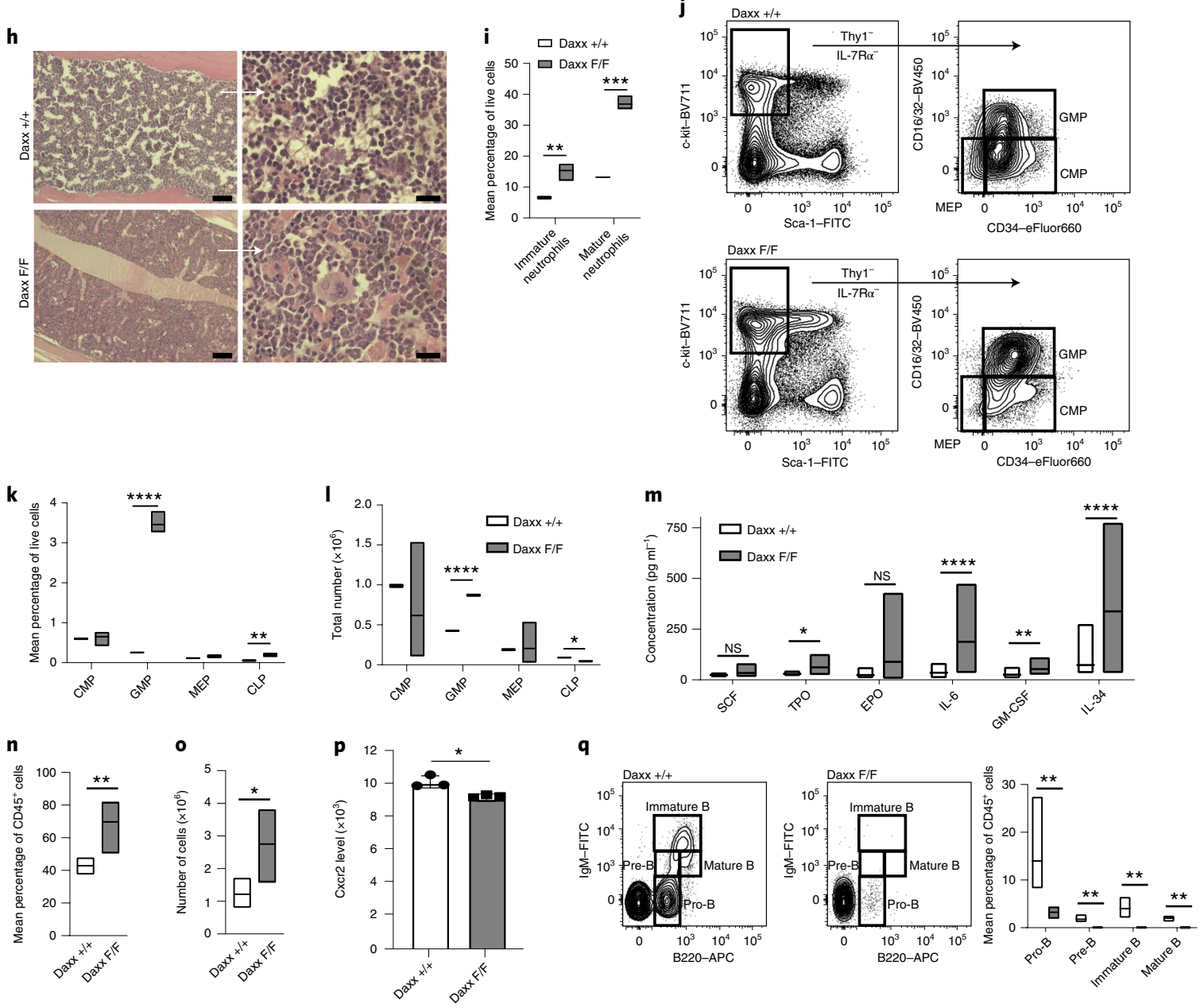


\section{Haematopoietic defects of Daxx deficiency are cell-intrinsic}

To determine the nature of the phenotypic changes described earlier, we performed colony-forming unit (CFU) assays using either $\mathrm{Lin}^{-}$progenitors (HPCs) or LT-HSCs from untreated $\operatorname{Daxx}^{\mathrm{F} / \mathrm{F}} ; \mathrm{Mx} 1 \mathrm{Cr} e^{+/-}$and $\mathrm{Daxx}^{+/+} ; \mathrm{M} \times 1 \mathrm{Cr}^{+/-}$mice, which underwent spontaneous recombination in vitro, probably due to activation of IFN signalling (not shown). An increase in colonies was observed following Daxx loss in HPCs (Extended Data Fig. 6a) at passage 0 (P0). Conversely, at P1 Daxx-deficient cells gave rise to fewer colonies, suggesting that their colony-forming potential was reduced. The CFU assays with LT-HSCs showed a similar phenotype following Daxx loss (Extended Data Fig. 6b,c), with an increased number of KLS cells at P0 and reduction at P1 (Extended Data Fig. 6d). Similar changes were observed in the number of $\mathrm{CD}_{11} \mathrm{~b}^{+}$cells and neutrophils (Extended Data Fig. 6e).

Finally, transplantation of BM cells isolated from pI:pC-treated mice into congenic recipients (Extended Data Fig. 6f) suggested a reduced reconstitution capacity of Daxx-KO stem/progenitor cells, resulting in a low frequency of CD $45.2^{+}$cells in the periphery (Extended Data Fig. 6g). Despite similar white-blood-cell counts (Extended Data Fig. 6h), KO transplants displayed altered frequencies of myeloid and B cells in the BM, PB and spleen, and of KLS cells and GMPs in the BM, as observed in the donor mice (Extended Data Fig. 6i-1).

Overall, these data suggest that the aberrant blood-cell composition observed following Daxx loss is due to a cell-intrinsic defect.

\section{Pu.1-driven myeloid signature following Daxx loss}

We performed transcriptome analyses of KLS and GMP subpopulations isolated from BM chimaeras (see the 'Haematopoietic defects of Daxx deficiency are cell-intrinsic' section; stress haematopoiesis) as well as from pI:pC-treated mice collected at 3 and 24 w.p.i. (Fig. 5a and Supplementary Tables 3-8; steady-state haematopoiesis). A PCA analysis revealed clustering according to genotype (Fig. 5a and Extended Data Fig. 7a) and that differences increased with time and following stress. When plotting the samples along pseudotime, Daxx-KO KLS cells followed a different trajectory, whereas KO GMPs did not (Fig. 5b and Extended Data Fig. 7b,c). Unsupervised clustering of selected master regulators and haematopoiesis markers revealed clustering according to genotype and condition as well as increased expression with time of myeloid-associated genes such as Mpo, Elane, Spil and Cebpa. This was accompanied by a reduction in the expression levels of lymphoid genes (Flt3, Ebf1, Irf4, Pax5 and Gfilb) in the KO KLS cells (Fig. 5c) but not in GMPs (Extended Data Fig. 7d). Accordingly, by comparing cells at 3 and 24 w.p.i. we found that Daxx-KO KLS cells switch from pathways compatible with increased lymphopoiesis to increased myelopoiesis and reduced B-cell generation (Fig. 5d,e and Extended Data Fig. 7e,f). This effect was even more pronounced in transplantation settings (Fig. 5 f and Extended Data Fig. 7 g). Furthermore, an increasing number of Pu.1-targetgenes driving myeloid fate were upregulated over time and following stress, accompanied by reduced expression of lymphoid Pu.1-target genes (Fig. 5g-i). These data are in accordance with neutrophilia becoming more pronounced with time (Fig. 4h), which was also confirmed by pathway analysis for Daxx-KO GMP cells (Extended Data Fig. 7h).

Transcriptomics of lymphoid- and myeloid-primed multipotent progenitors (MPP4 and MPP3, respectively) combined with unsupervised clustering of myeloid marker gene and transcription factor expression showed that Daxx-KO MPP4 cells cluster together with WT MPP3 (Fig. 5j, top). A similar analysis based on lymphoid genes showed Daxx-KO MPP4 clustering with MPP3 cells, suggesting that Daxx-KO lymphoid-biased MPP4 have shifted towards a more myeloid-biased gene expression (Fig. 5j, bottom).

Deregulation of ERVs/RTEs in Daxx-KO KLS cells and GMPs became more pronounced with time and following stress, especially that of ERV1 and IAPs (Fig. 5k and Extended Data Fig. 7i). These changes correlated with induction of ISGs and RNA-sensing factors in both Daxx-KO KLS and GMPs, mostly at 24 w.p.i., and in transplanted cells (Fig. 51 and Extended Data Fig. 7j). Spleen staining showed increased levels of the retinoic acid-inducible gene I-pathway (RIG-I) component melanoma differentiation-associated gene 5 (Mda5) and double-stranded RNA (dsRNA; Extended Data Fig. 8a). Furthermore, TERRA long-noncoding RNA (lncRNA) was upregulated in Daxx-KO BM cells (Extended Data Fig. 8b) and there was significant overlap between differentially expressed genes (DEGs) from TERRA knockdown in embryonic stem cells $^{23}$ with DEGs in Daxx-KO versus WT GMP datasets (Extended Data Fig. 8c).

Finally, ERV-overlapping enhancers in proximity of both up- and downregulated genes were more accessible in Daxx-KO KLS cells at 3 w.p.i. (Fig. $5 \mathrm{~m}, \mathrm{n}$ ). For instance, Spil and Gfil (which together drive neutrophilic differentiation ${ }^{40}$ ) displayed increased enhancer accessibility at 3 w.p.i. but were upregulated only in transplantation settings. In contrast, Irf4 expression (which cooperates with Pu.1 for B-cell differentiation ${ }^{41}$ ) was higher at 3 w.p.i. but lower at 24 w.p.i. and in transplantation settings.

\section{Daxx loss alters H3.3, Pu.1 and histone-mark distribution}

We next performed low-input chromatin immunoprecipitation combined with sequencing using Cleavage Under Targets and Tagmentation (CUT\&Tag) for H3.3, Pu.1, H3 lysine 27 acetylation (H3K27ac) and H3K9 trimethylation (H3K9me3) on WT and Daxx-KO HSPCs at 3 w.p.i. Daxx loss led to significant changes in H3.3 distribution and Pu.1 binding (Fig. 6a). Reduction in both H3.3 and Pu.1 at distal regions, particularly at ERVs, was accompanied by enrichment of both proteins at promoters (Fig. 6a), suggesting that their respective chromatin associations may be linked. H3.3-depleted enhancers were characterized by increased chromatin accessibility and H3K27ac but decreased Pu.1 levels (Fig. 6b,c). In turn, nearby genes showed increased Pu.1 binding around and downstream of the transcription start site (TSS), coinciding with increased chromatin accessibility and H3.3 enrichment (Fig. 6c). Pathway analysis for genes close to distal regions with altered $\mathrm{Pu} .1$ binding showed enrichment of immune-cell categories (Fig. 6d).

Fig. 3 | Daxx-deficient spleens display marked structural changes linked to an increase of neutrophils and reduction of B cells. a, Representative images of H\&E-stained spleen sections ( $n=3$ independent experiments). Scale bars, $100 \mu \mathrm{m}$ (left) and $20 \mu \mathrm{m}$ (middle; higher-magnification images). Overview images of the section are shown (right). $\mathbf{b}$, Total number of spleen cells ( $n=3$ mice per genotype). $\mathbf{c}$, Flow cytometry analysis of cell surface markers ( $n=6$ mice per genotype). d, Flow cytometry plots showing macrophage- and monocyte-like populations. e, Frequencies of neutrophils (left) and eosinophils (right; $n=5$ mice per genotype). $\mathbf{f}$, Cxcr2 levels in mature neutrophils of the spleen ( $n=3$ mice per genotype). MFU, mean fluorescent units. $\mathbf{g}$, Total B-cell and neutrophil counts in the spleen ( $n=5$ mice per genotype). $\mathbf{h}$, Frequencies of follicular and marginal-zone B cells (FOB and MZB, respectively; $n=6$ mice per genotype). $\mathbf{i}$, Levels of F4/80 (left), CD11b (middle) and combined F4/80 and CD11b (right) fluorescence ( $n=3$ mice per genotype). $\mathbf{j}$, Immunofluorescence images of spleen sections ( $n=3$ mice per genotype). Scale bars, $100 \mu \mathrm{m}$. $\mathbf{k}$, Magnified views of the images in $\mathbf{j}$. Scale bars, $15 \mu \mathrm{m}$. $\mathbf{c}, \mathbf{e}, \mathbf{g}, \mathbf{h}$, Boxplots show the minimum and maximum values (box boundaries) and the mean (horizontal line). $\mathbf{b}, \mathbf{f}, \mathbf{i}$, Data are the mean \pm s.d. ${ }^{\star} P<0.05$, ${ }^{\star \star} P<0.01$ and NS, not significant; Student's $t$-test $(\mathbf{b}, \mathbf{f}, \mathbf{g}, \mathbf{i})$ or non-parametric Wilcoxon rank test $(\mathbf{c}, \mathbf{e}, \mathbf{h})$. Daxx F/F, Daxx KO and Daxx +/+, Daxx WT. Exact $P$ values and numerical source data are provided. 

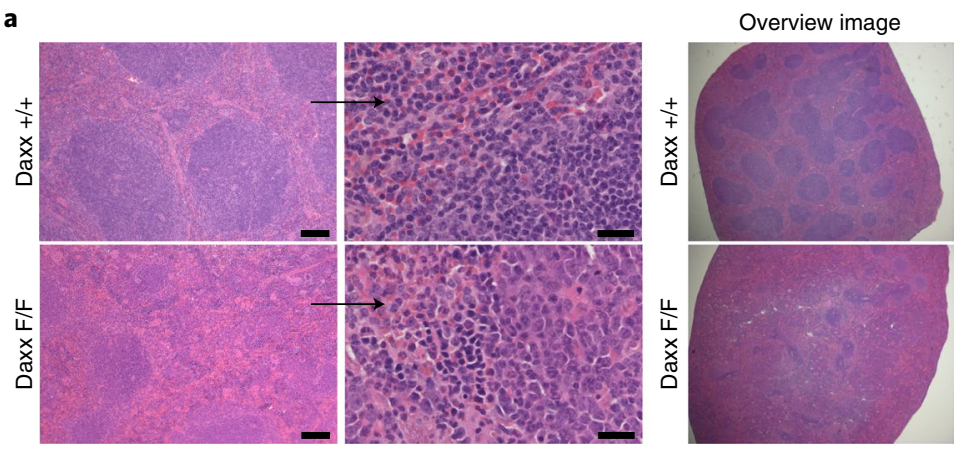

b

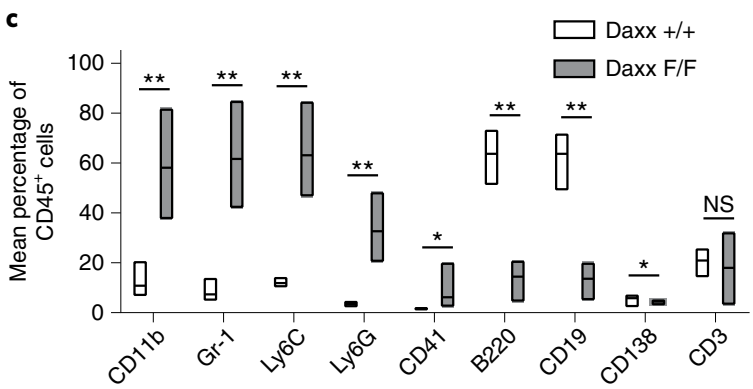

d

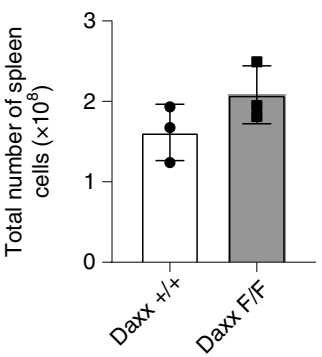

Gating: CD11b ${ }^{+}$CD11C ${ }^{-}$Ly6G $^{-}$SSC $^{10}$
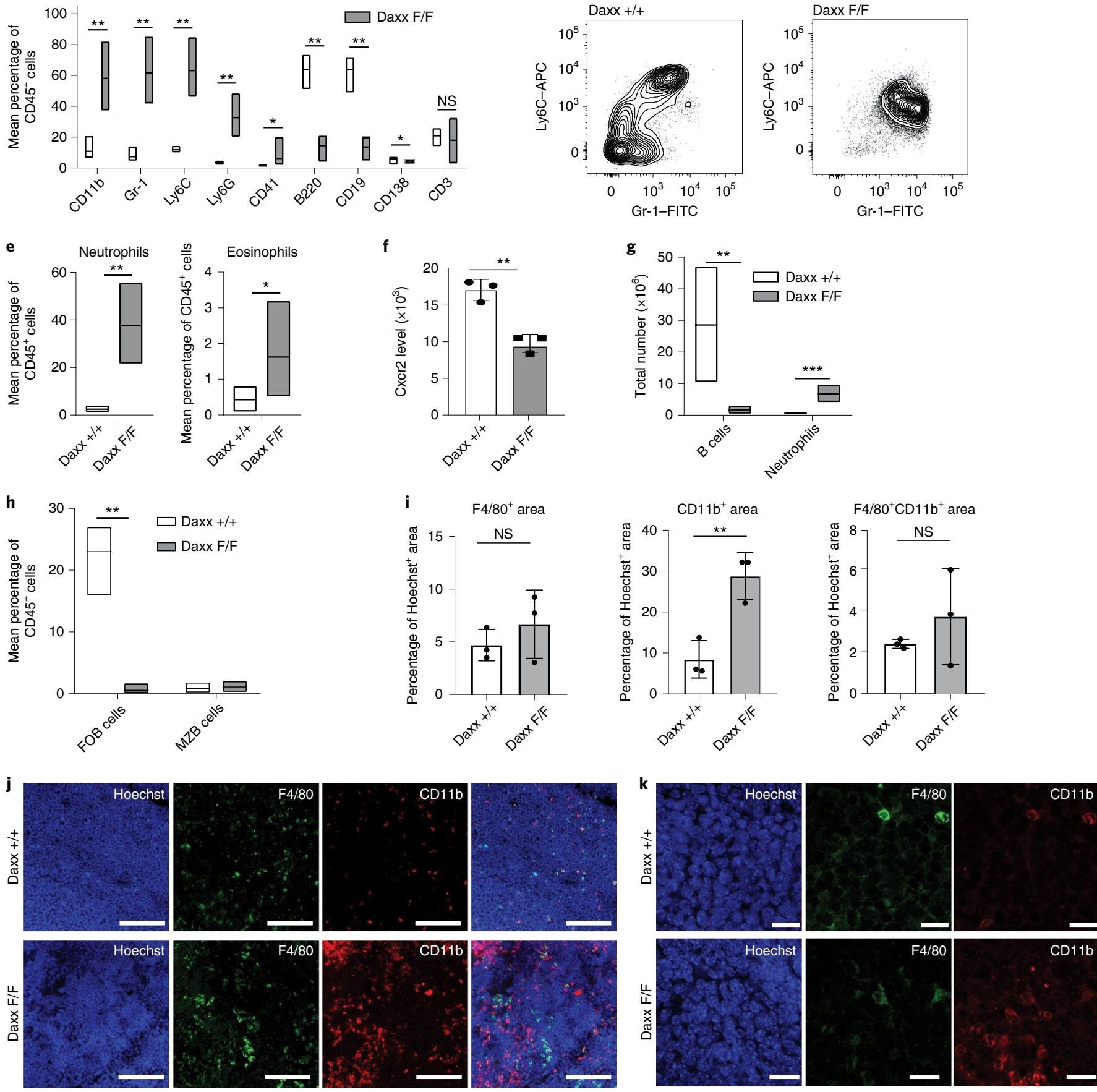
In addition, altered Pu.1 binding at Pu.1-regulated transcription factors (Fig. 6e) corresponded to changes in their gene expression (Fig. 5g).
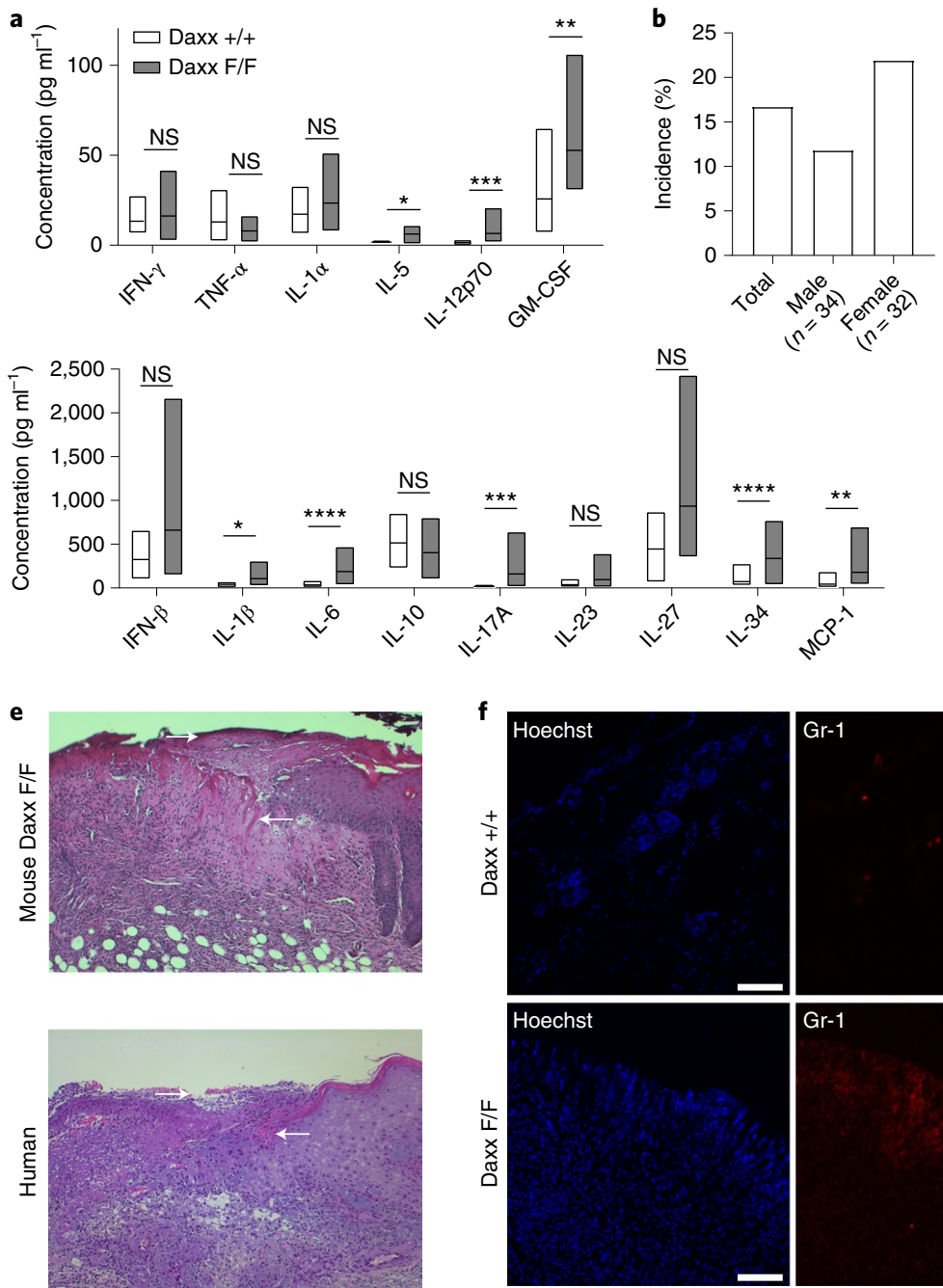

h
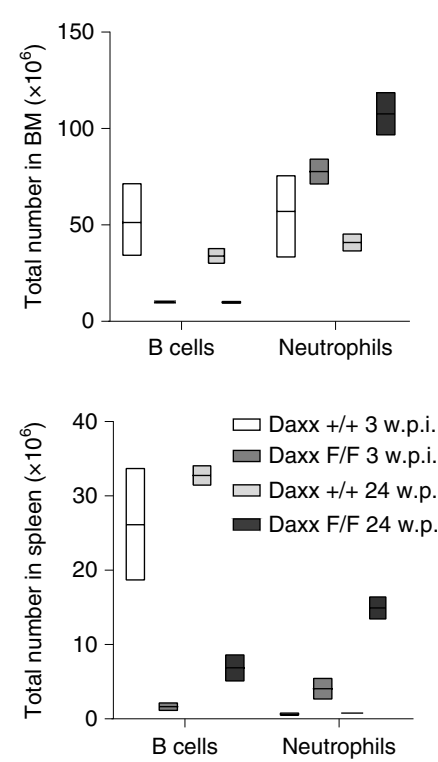
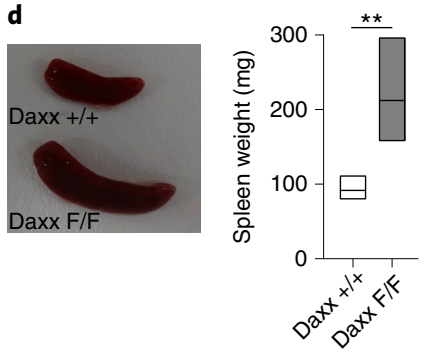

c

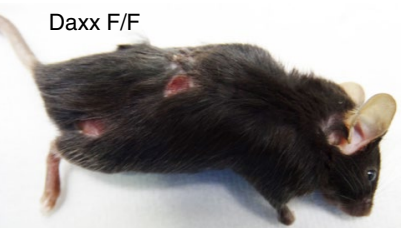

$a^{+x^{+}} y^{+x}$
Enhancers overlapping with ERVs/RTEs showed stronger depletion of $\mathrm{H} 3 \mathrm{~K} 9 \mathrm{me} 3$ and increase in $\mathrm{H} 3 \mathrm{~K} 27 \mathrm{ac}$ than other enhancers (Fig. 6f), despite a similar increase in chromatin 
Fig. 4 | Chronic Daxx loss leads to increased inflammatory cytokines and pyoderma gangrenosum, an autoinflammatory neutrophilic disease. $\mathbf{a}$, Concentrations of cytokines in plasma ( $n=6$ mice per genotype). $\mathbf{b}$, Incidence of skin lesions in the mice in the total group and stratified by sex. Data are the mean. $\mathbf{c}, \operatorname{Daxx}{ }^{\mathrm{F} F} ; \mathrm{Mx} 1 \mathrm{Cre}^{+/-}$mouse with skin lesions. d, Representative image of the spleens of control and Daxx-KO mice with skin lesions (left), and the spleen weight of mice with skin lesions (right; $n=6$ mice per genotype). e, Skin lesion of a Daxx ${ }^{\mathrm{F} / \mathrm{F}} M \mathrm{Mx} \mathrm{Cre}{ }^{+/-}$mouse (top; $n=3$ biological replicates) compared with a human skin biopsy of a patient with pyoderma gangrenosum (bottom). Representative images of H\&E-stained sections; the arrows indicate the site of the ulcer and fibrin layer. $\mathbf{f}$, Immunofluorescence staining of $\mathrm{Gr}-1^{+}$cells in sections of skin lesions ( $n=2$ independent experiments). Scale bars, $100 \mu \mathrm{m} . \mathbf{g}$, Immunofluorescence staining of sections of skin lesions with citH3 ( $n=2$ independent experiments). The higher-magnification image (bottom) shows NETosis in a Daxx ${ }^{\mathrm{F} / \mathrm{F}} ; \mathrm{Mx}_{1 \mathrm{Cre}}{ }^{+/-}$skin lesion. Scale bars, $100 \mu \mathrm{m}$ and $20 \mu \mathrm{m}$ (higher-magnification image). $\mathbf{h}$, Number of B cells and neutrophils in the BM (top) and spleen (bottom) at 3 and 24 w.p.i. ( $n=2$ mice per genotype, except for WT at 3 w.p.i., where $n=4$ mice). a,h, Boxplots show the minimum and maximum values (box boundaries) and the mean (horizontal line). ${ }^{\star} P<0.05,{ }^{\star \star} P<0.01,{ }^{\star \star \star} P<0.001,{ }^{\star \star \star \star} P<0.0001$ and NS, not significant; non-parametric Wilcoxon rank test. Daxx F/F, Daxx KO and Daxx $+/+$, Daxx WT. Exact $P$ values and numerical source data are provided.

accessibility. Generally, ERVs/RTEs showing increased accessibility were enriched in $\mathrm{H} 3 \mathrm{~K} 27 \mathrm{ac}$ but displayed minor changes in H3K9me3 (Fig. 6g). Finally, among enhancers (Fig. 6h) and ERVs/ RTEs gaining H3K27ac (Fig. 6i), one subset displayed clear reduction in H3.3 following Daxx loss.

Together, these findings suggest that Daxx loss alters chromatin features and Pu.1 distribution at enhancers, including those overlapping with ERVs.

\section{Phenotypic rescue and unique changes after Daxx and Pu.1 DKO}

We next investigated whether the phenotypic alterations caused by Daxx loss were dependent on Pu.1 by using a Spi1 ${ }^{\mathrm{FF}}$ line $e^{42}$. The BM cellularity was not significantly different in Daxx-KO mice compared with Daxx and Pu.1 double-KO (DKO) mice, although the latter displayed a tendency towards higher cellularity (Fig. 7a). At 8 w.p.i., the BM of the DKO mice displayed significant reduction in the GMP and neutrophil frequencies and numbers compared with the Daxx-KO (Fig. 7b,c), whereas the B220+ cells in the BM were still compromised (Fig. 7c). Conversely, in DKO spleens, the $\mathrm{B}$-cell and neutrophil frequencies and numbers were normalized (Fig. $7 \mathrm{~d}-\mathrm{g}$ ) along with spleen architecture and selected cytokines (at 3 and 8 w.p.i.; Fig. 7 h-j). The B-cell frequency was also partially rescued in the DKO PB (Fig. 7k,l). Neutrophil and B-cell numbers and frequencies in the BM, spleen and PB were similar to WT following the loss of Pu.1 alone (Extended Data Fig. 8d,e).

To link the phenotypic rescue to normalization of molecular perturbations, we studied changes in transcription, chromatin landscape and histone marks in single-KO and DKO mice (Supplementary Tables 9 and 10). The PCA analysis of RNA-seq data showed that each genotype clustered separately (Fig. 8a,b). Although DEGs from different comparisons (Daxx-KO versus WT, DKO versus WT and Pu.1-KO versus WT) partially overlapped, many genotype-specific changes also occurred, particularly in KLS cells (Extended Data Fig. 9a,b). Pathway analysis based on DKO-specific and Pu.1-KO-specific DEGs in KLS cells and GMPs identified distinct biological functions and diseases, including some associated with lymphoid neoplasms (Fig. 8c,d and Extended Data Fig. 9c,d). Clustering of expression for haematopoietic transcription factors and markers, Pu.1-target genes, ISGs and the dsRNA-sensing machinery revealed both common and genotype-specific changes between Pu.1-KO and DKO cells (Extended Data Fig. 9e-g). Altered expression of selected ERV/RTE subtypes in Daxx-KO KLS cells and GMPs were in part reverted in the DKO cells (Fig. 8e,f). Finally, Pu.1-KO KLS cells and GMPs showed upregulation of some ERV/RTE subtypes and satellite repeats, suggesting a role for Pu.1 in their regulation.

On investigation of the chromatin changes, we found that $90.8 \%$ of distal regions that opened following Daxx loss became closed in DKO cells (Fig. 8g). Conversely, the vast majority of distal regions found closed in the Daxx-KO KLS cells opened in DKO KLS cells. In addition, we found DKO-specific chromatin changes: 15,587 distal regions that were open in WT and Daxx-KO KLS cells became closed in DKO cells, while 3,922 distal sites that opened in DKO cells were closed in the other genotypes. When we compared open regions in WT, Daxx-KO and DKO KLS cells, we found that DKO cells generally displayed the lowest accessibility (Fig. 8h). Many of the genes that were significantly upregulated in the Daxx-KO KLS cells at 3 w.p.i. and resided near enhancers with increased accessibility were downregulated in the DKO cells (Fig. 8i). Finally, opening of TERRA-BS at sex chromosomes in Daxx-KO KLS cells was reverted in DKO cells (Extended Data Fig. 10a). Closing of chromatin at the TERRA-BS in the vicinity of the Mid1 and Erdr1 genes in the DKO cells correlated with rescued gene expression compared with the Daxx-KO cells (Extended Data Fig. 10b).

H3K9me3 alterations found in Daxx-KO HSPCs were reverted to some extent in DKO cells (Fig. 8j). In addition, we found that there were more distal regions in DKO HSPCs that gained H3K9me3

Fig. 5 | Daxx loss affects the transcriptome of haematopoietic progenitors in the context of both steady-state and stress haematopoiesis. RNA-seq analysis of $\mathrm{Daxx}^{+++} ; \mathrm{M} \times 1 \mathrm{Cre}^{+/-}$and $\mathrm{Daxx}{ }^{\mathrm{F} / \mathrm{F}} ; \mathrm{Mx} 1 \mathrm{Cre} \mathrm{r}^{+/-} \mathrm{KLS}$ cells isolated at 3 and 24 w.p.i. or from transplanted animals. a, Experimental scheme for cells isolated from pl:pC-treated mice (top) and PCA of the top-500 most variable genes (bottom). $\mathbf{b}$, Mean pseudotime for each sample group ( $n=2$ mice per genotype). c, Heatmap showing scaled expression of selected transcription factors and regulators involved in blood-cell differentiation ( $n=2$ mice per genotype). d, Summary plot of IPA analysis of DEGs at 3 w.p.i. e, Summary plot of IPA analysis of DEGs at 24 w.p.i. f, Summary plot of IPA analysis of DEGs in KLS cells from transplanted animals. $\mathbf{d}-\mathbf{f}$, Predicted activation of protein or biofunction is indicated in orange and predicted inhibition of the displayed protein or biofunction in dark and light blue, respectively. Grey dotted lines are machine learning-based inferred connections. $\mathbf{g}$, Expression changes, at 3 w.p.i., of the transcription factor genes regulated by Pu.1. h, Expression changes, at 24 w.p.i., of transcription factor genes regulated by Pu.1. i, Expression changes of the transcription factor genes regulated by Pu. 1 in KLS cells isolated from transplanted mice. $\mathbf{g}$-i, Upregulated genes are shown in red and downregulated genes in green. A stronger colour intensity indicates higher absolute $\log _{2}$-transformed fold changes. $\mathbf{j}$, Heatmaps showing myeloid- or lymphoid-driving transcription factors for MPP3 and MPP4 cells. k, Normalized read counts of ERV/RTE subtypes and satellite repeats ( $n=2$ mice per genotype). I, Heatmap showing expression of interferon-responsive genes and dsRNA-recognition machinery. $\mathbf{m}$, ATAC-seq read coverage at enhancers overlapping ERVs close to genes that are up- or downregulated in KLS cells collected at 3 and 24 w.p.i. or from transplanted animals. $\mathbf{n}$, Genome browser tracks of the regulatory regions of SpiT, Irf4 and Gfil showing ATAC-seq and RNA-seq coverage. Transcripts of genes of the depicted region are shown below. b,k, Boxplots show the minimum and maximum values (box boundaries) and the mean (horizontal line). Daxx F/F, Daxx KO and Daxx $+/+$, Daxx WT. Numerical source data provided. 
a

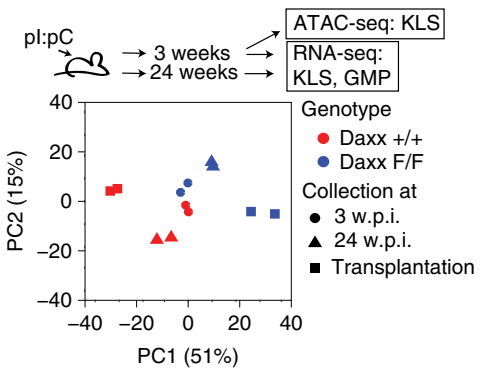

d

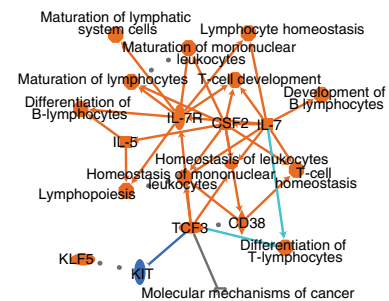

g

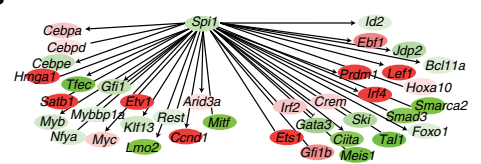

j

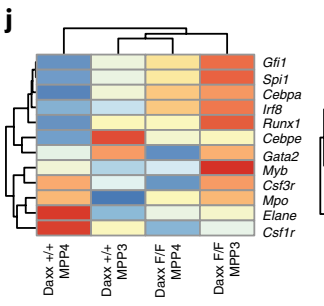

I

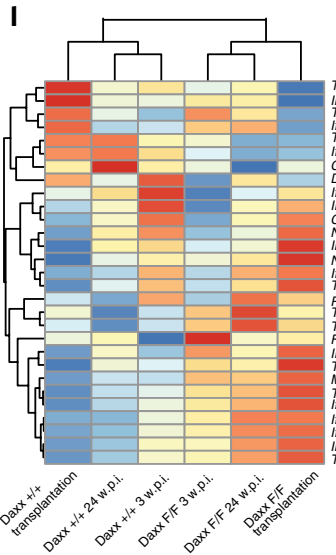

b

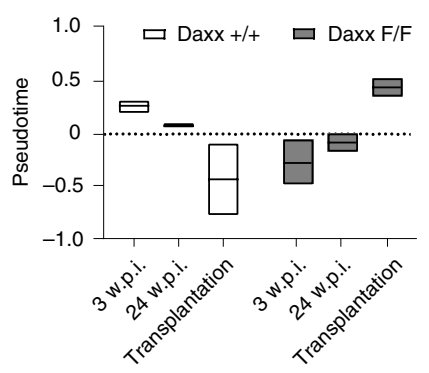

e

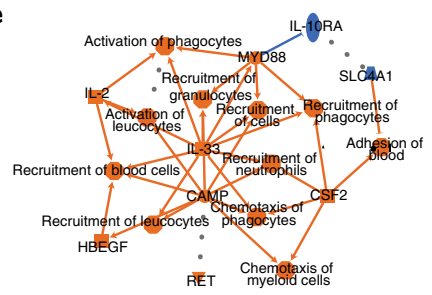

h

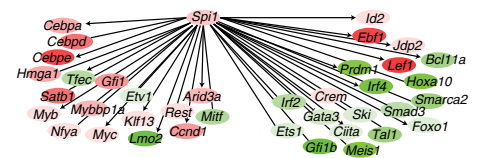

k

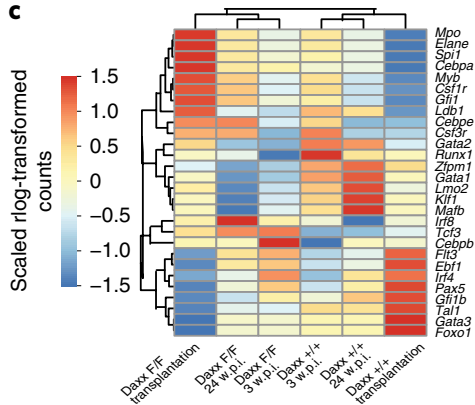

f

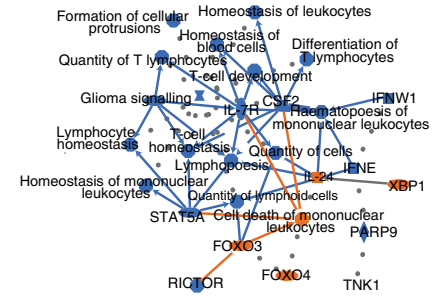

i

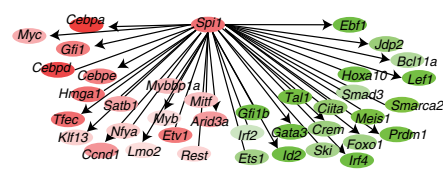

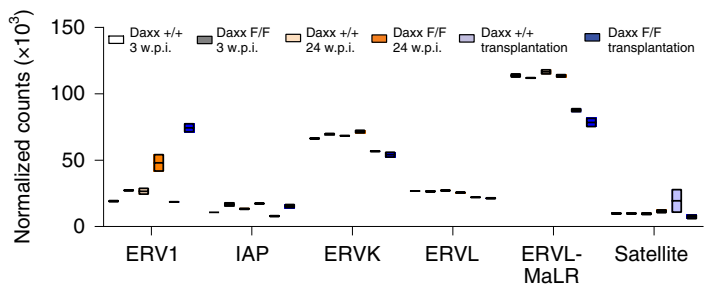
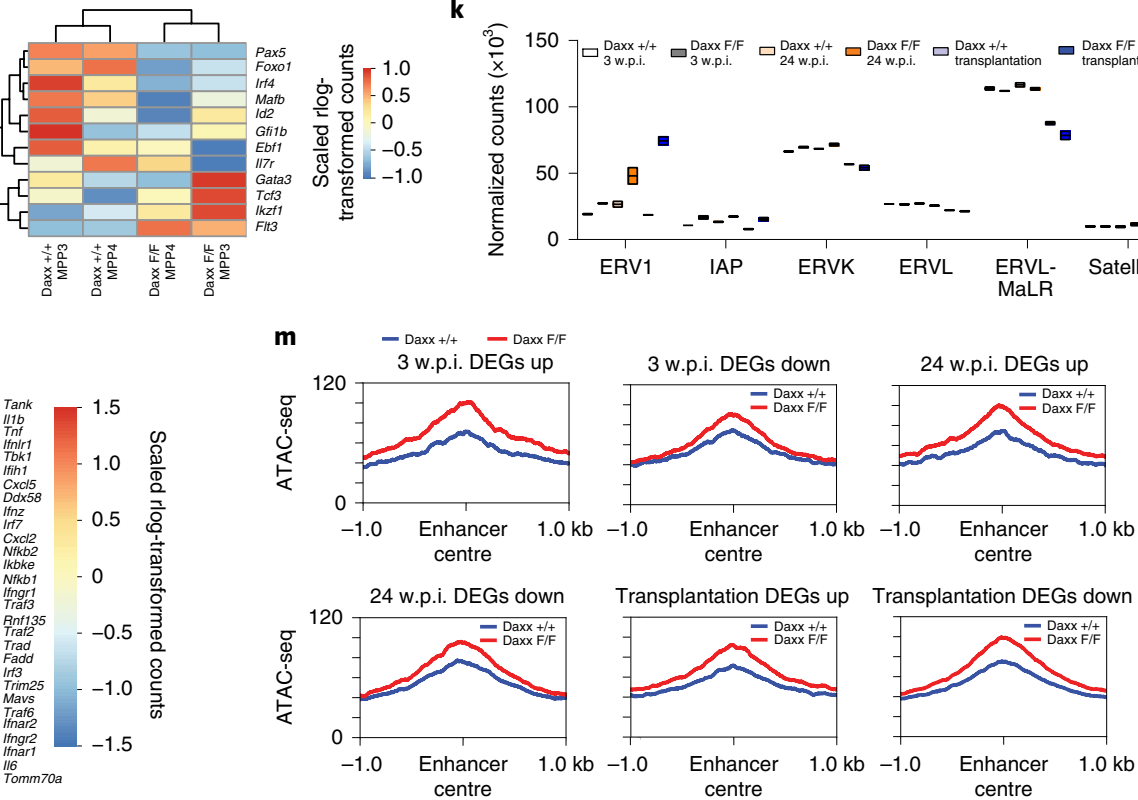

Transplantation DEGs down

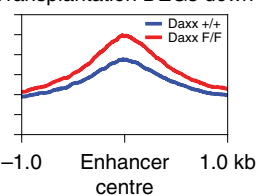

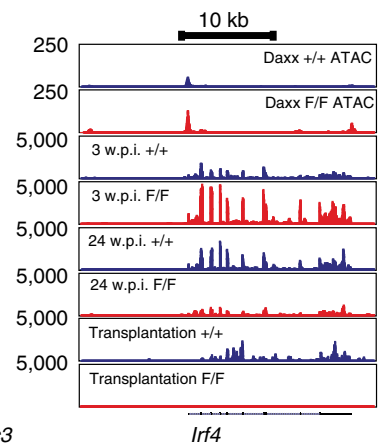

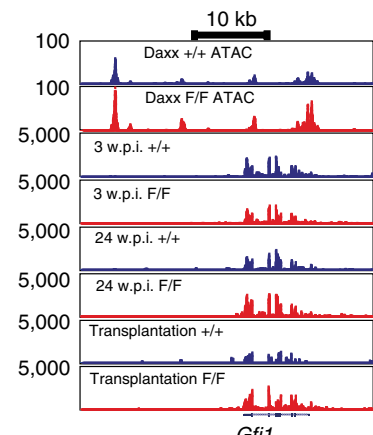



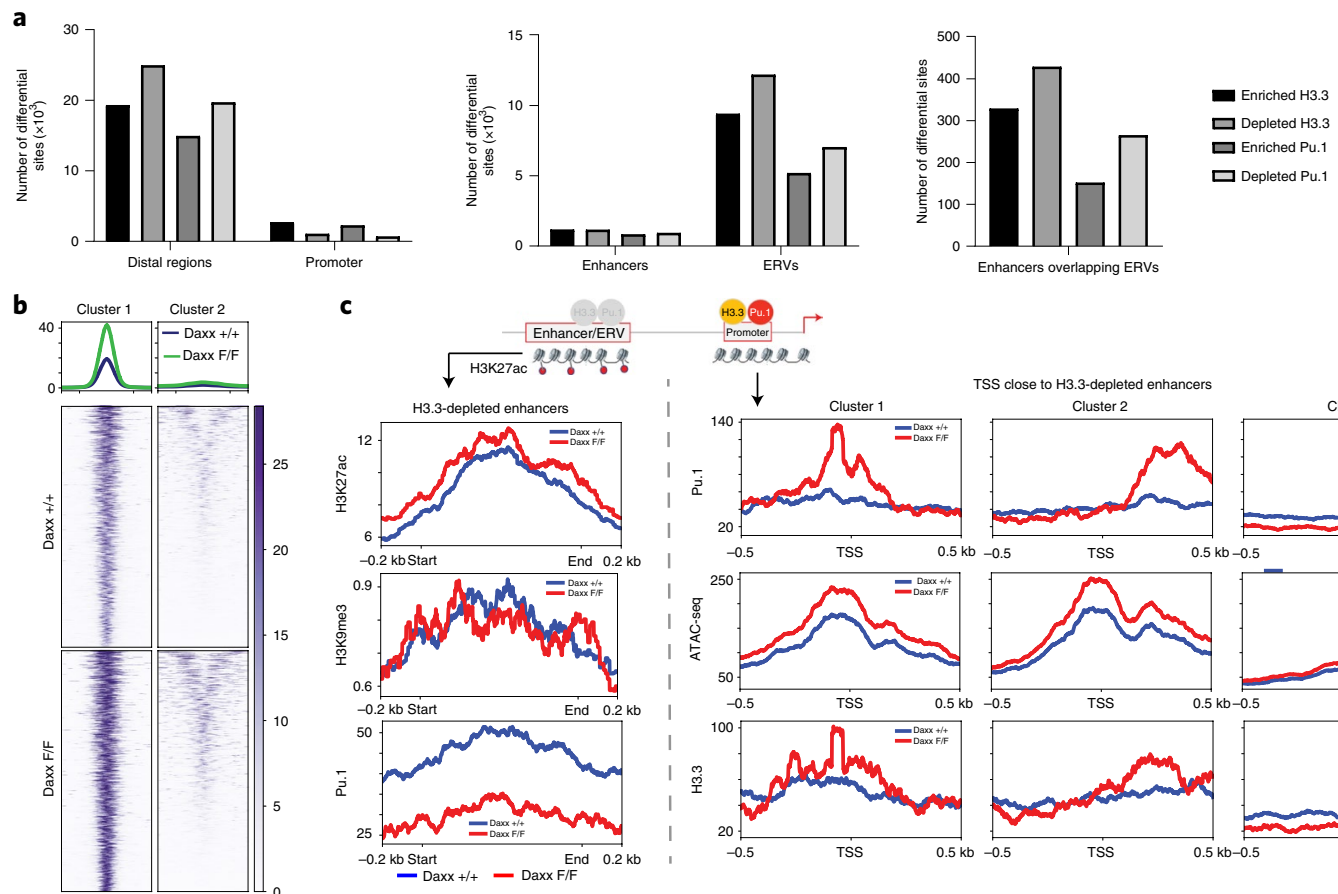

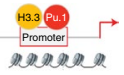

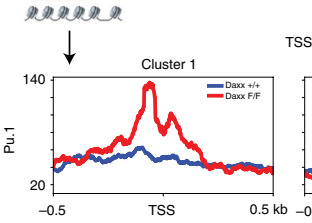

TSS close to H3.3-depleted enhancers
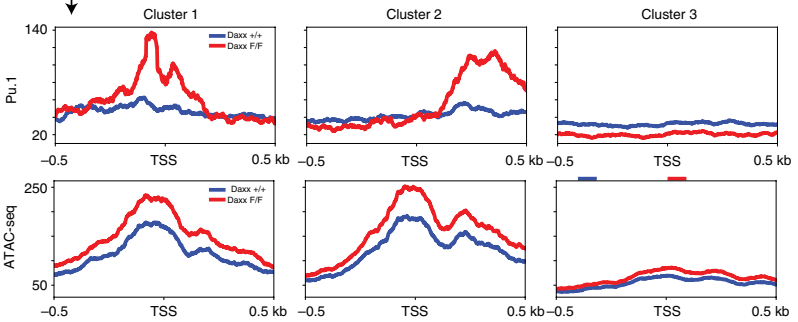

d Cardiac hypertrophy signalling (enhanced)
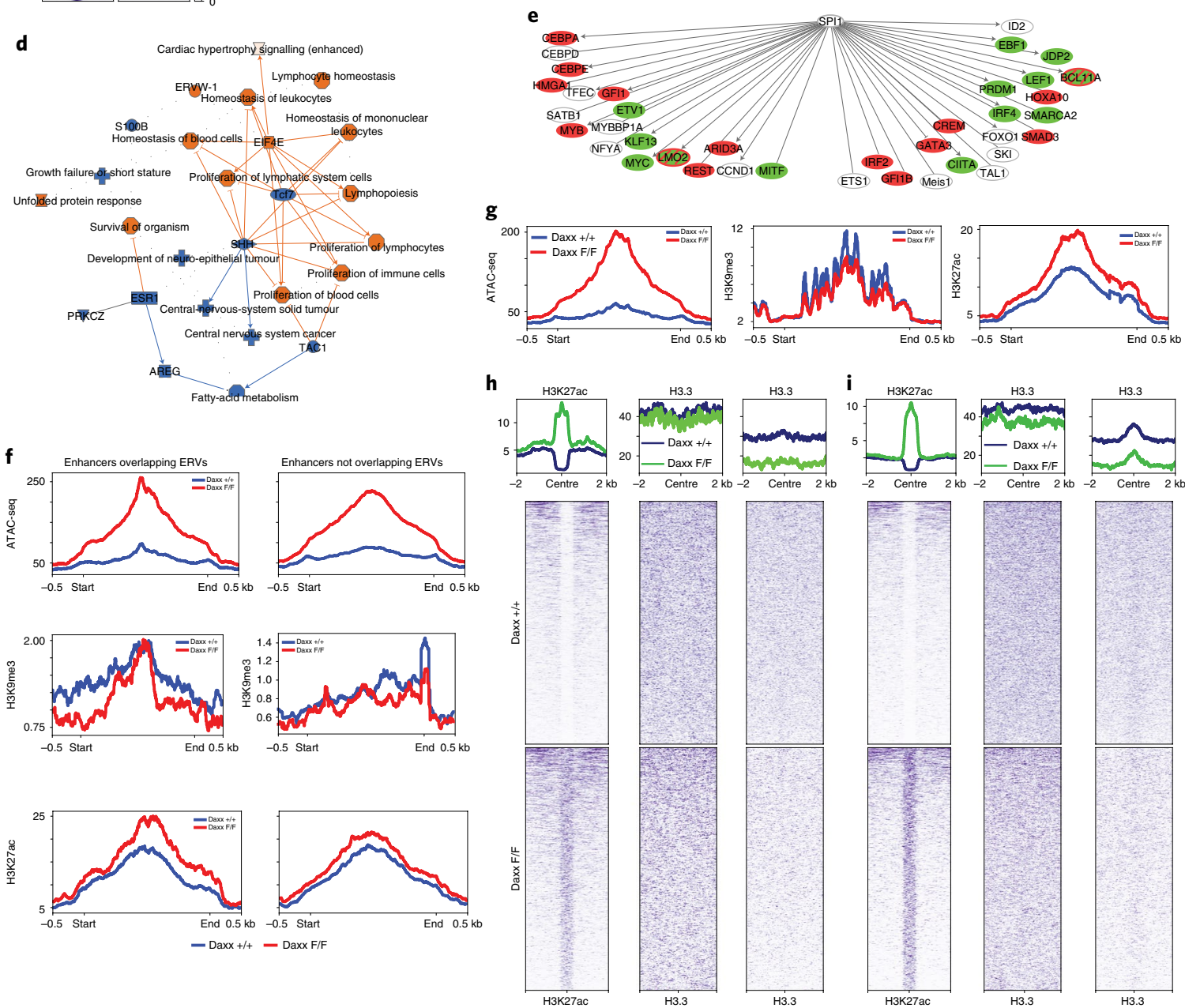

$(7,862)$ than those with reduced H3K9me3 $(1,147)$ compared with WT or Daxx-KO HSPCs. We also observed that the overall levels of H3.3 were markedly increased in DKO cells at enhancers and ERVs compared with both the WT and Daxx-KO cells (Fig. 8k).
Finally, we zoomed in on the locus encoding Fbp1 and Fbp2 (Fig. 81), two critical regulators of gluconeogenesis that affect HSPC repopulation capacity via inhibition of glycolysis under the control of Setdb1 (ref. ${ }^{43}$ ). Fbp1 was upregulated in Daxx-KO KLS 
Fig. 6 | Daxx-deficient progenitors display alterations in H3.3 and Pu.1 genome-wide distribution as well as epigenetic marks. a, Overview of altered H3.3 distribution and Pu.1 binding in Daxx-KO HSPCs determined by CUT\&Tag assays. b, Heatmaps of ATAC-seq read distribution around the centre of H3.3-depleted enhancers. c, H3K27ac, H3K9me3 and Pu.1 CUT\&Tag read distribution across H3.3-depleted enhancers (left) as well as Pu.1, ATAC-seq and $\mathrm{H} 3.3$ coverage around the TSS of genes close to H3.3-depleted enhancers (right). Pu.1 coverage was stratified into three clusters by $k$-means and ATAC-seq read distribution was plotted for the same clusters of TSS. d, Summary plot of IPA analysis for genes close to distal regions with altered Pu.1 binding. Predicted activation of the protein or biofunction is indicated in orange and predicted inhibition of the displayed protein or biofunction in blue. e, Graphical depiction of Pu.1-binding changes at distal or proximal sites close to transcription factors regulated by Pu.1. The regions with increased Pu.1 binding are shown in red and regions with decreased Pu.1 binding in green. f, Enrichment plots for ATAC-seq, H3K9me3 CUT\&Tag and H3K27ac CUT\&Tag at enhancers overlapping and not overlapping ERVs with increased accessibility. g, Enrichment plots for ATAC-seq, H3K9me3 CUT\&Tag and H3K27ac CUT\&Tag at ERVs with increased accessibility. h, Heatmaps of enhancers with increased H3K27ac showing cluster analysis with H3.3. i, Heatmaps of ERVs with increased H3K27ac showing cluster analysis with H3.3. h,i, The legend for the heatmaps is the same as $\mathbf{b}$ and middle graphs show cluster of enhancers or ERVs/RTEs with no difference in H3.3 while right graphs show those with reduced H3.3 loading. Daxx F/F, Daxx KO and Daxx +/+, Daxx WT. Numerical source data are provided.

cells, whereas $F b p 2$ remained silenced apart from transplantation settings. Fbp1 silencing was restored in DKO cells, suggesting that $\mathrm{Pu} .1$ is required for its induction. This is in accordance with three main regions bound by Pu. 1 at this locus (as reported in ${ }^{19}$ ), two of which coincide with haematopoietic enhancers ${ }^{18}$. H3K27ac enrichment at these regions in Daxx-KO cells was reverted in DKO cells. Although H3.3 was reduced at the proximal enhancer in Daxx-KO cells, its levels were restored in DKO cells, suggesting involvement of another chaperone, such as Hira. A similar trend applied to $\mathrm{H} 3 \mathrm{~K} 9 \mathrm{me} 3$ at this enhancer. The large block of $\mathrm{H} 3 \mathrm{~K} 9 \mathrm{me} 3$ over the $F b p 2$ gene and its regulatory regions, which was previously linked to Fbp2 upregulation in $\mathrm{HSPCs}^{43}$ following Setdb1 loss, was further enriched in Daxx-KO cells but not DKO cells. Finally, H3K27me3 was substantially reduced across the entire locus in the Daxx-KO and DKO cells, especially at the most proximal enhancer of $F b p 1$, suggesting that this mark may cooperate with Pu.1 loss for Fbp1 silencing.

\section{Discussion}

Together, our findings implicate the H3.3 chaperone and ERV/RTE repressor Daxx in the regulation of haematopoiesis and protection from inflammation. We propose that Daxx acts as an epigenetic barrier controlling cell plasticity in HSPCs in part via RTE control, in turn ensuring balanced cell differentiation (Extended Data Fig. 10c). Recent work in zebrafish implicates RTE-driven engagement of RNA-sensing in HSPC emergence in haematopoiesis ${ }^{44}$. In this respect, we showed that ERV/RTE induction correlates with the IFN type I-like response in Daxx-deficient LT-HSCs. IFN type I signalling is normally repressed in HSCs, as it may promote cell death ${ }^{45}$, but when activated in quiescent HSCs it causes cell-cycle entry $^{46}$. Following acute Daxx loss, the number of LT-HSCs and other progenitor types did indeed increase. As it is unlikely that stem/progenitor cells are able to produce IFNs, ISG induction could be IFN-independent as reported in viral-infection model $s^{4-50}$. This could explain the moderate ISG induction in Daxx-KO LT-HSCs.
Furthermore, it is possible that ERV/RTE derepression following Daxx loss may impair B-cell differentiation, as observed in mice lacking the ERV/RTE-silencing Setdb1-Kap1 complex, a Daxx interactor $^{51-55}$.

Our study points to a link between Daxx and the pioneer transcription factor Pu.1. Pu.1 engagement does not seem to be an immediate effect of Daxx loss in HSCs. We instead observed progressive activation of a Pu.1 myeloid programme in haematopoietic progenitors with time and following stress, along with aggravation of neutrophilia. This mirrors the response to viral infections, where HSC activation is followed by a return to quiescence and an accumulation of myeloid-biased $\mathrm{HSCs}^{56}$, a phenomenon that has also been observed in ageing ${ }^{57}$. Thus, Pu.1 may contribute to the myeloid bias within a subset of Daxx-deficient HSCs that have returned to quiescence.

Concomitant Daxx and Pu.1 loss reverts some phenotypic perturbations found in Daxx-KO mice, including a partial recovery of B-cell differentiation and spleen architecture. This would be in agreement with the reported role of Pu.1 in inhibition of terminal $\mathrm{B}$-cell and plasma-cell differentiation ${ }^{58}$. At the molecular level, the increase in enhancer accessibility that typifies Daxx-KO KLS cells is reverted by over $90 \%$ in DKO cells. Closing of chromatin in DKO cells at TERRA-BS correlated with rescued gene expression of neighbouring genes, suggesting that perturbations at TERRA-BS and its target genes, such as Erdr1, may contribute to the phenotypes caused by Daxx loss ${ }^{26}$. However, DKO progenitors also show unique chromatin and transcriptome alterations that are not found in single $\mathrm{Pu}$.1- or Daxx-KO mice, suggesting a genetic interaction between the two pathways. In support of this hypothesis, Daxx loss causes reduction in the levels of both $\mathrm{Pu} .1$ and $\mathrm{H} 3.3$ at enhancers, correlating with their increased enrichment at neighbouring genes. These findings suggest that Pu.1 and H3.3 may influence their reciprocal genome distribution.

Finally, dysfunction of intrinsic immunity mechanisms devoted to ERV/RTE silencing may predispose to inflammation and neoplastic

Fig. 7 | Concomitant loss of Daxx and Pu.1 partially restores lymphopoiesis while suppressing peripheral accumulation of neutrophils. a, Total BM cell counts ( $n=3$ mice per genotype) in four leg bones per animal. b. Percentage (left) and number (right) of CMPs and GMPs in the BM ( $n=5$ mice per genotype). c, Percentage (left) and number of B cells and neutrophils in the BM ( $n=5$ mice per genotype). d, Total spleen cell counts ( $n=3$ mice per genotype). e, Percentage (left) and number (right) of B cells and neutrophils in the spleen ( $n=5$ mice per genotype). f, Flow cytometry plots showing B and T cell-like populations in the spleen. $\mathbf{g}$, Flow cytometry analysis showing neutrophil- and monocyte-like populations in the spleen. $\mathbf{f}, \mathbf{g}$, The percentages of cells in the gated regions of the plots are indicated. $\mathbf{h}$, Representative immunofluorescence overview images of spleens stained with 4,6-diamidino2-phenylindole (DAPI) and B220 ( $n=2$ independent experiments). Scale bars, $500 \mu \mathrm{m}$. i, Inflammatory cytokine levels in plasma at 3 w.p.i. ( $n=5 \mathrm{WT}$ and 2 Daxx-KO and DKO mice). j, Inflammatory cytokine levels in plasma at 8 w.p.i. ( $n=5$ mice per genotype). $\mathbf{k}$, Percentage of $B$ cells and neutrophils in the PB ( $n=5$ mice per genotype). I, Flow cytometry plots showing B and T cell-like populations in PB ( $n=5$ mice per genotype). a,d, Data are the mean \pm s.d. $\mathbf{b}, \mathbf{c}, \mathbf{e}, \mathbf{k}-\mathbf{j}$, Boxplots show the minimum and maximum values (box boundaries) and the mean (horizontal line). ${ }^{\star} P<0.05,{ }^{\star \star} P<0.01,{ }^{\star \star \star} P<0.001$ and NS, not significant; Student's t-test. Daxx F/F, Daxx KO and Daxx +/+, Daxx WT; Pu.1 F/F, Pu.1-KO and Pu.1 +/+, Pu.1 WT. Exact $P$ values and numerical source data are provided. 
b

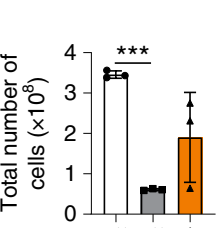

$\sqrt{x+1}, x_{x}<x$

Qu. 0.00.

$x \times<<<<$

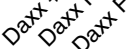

e
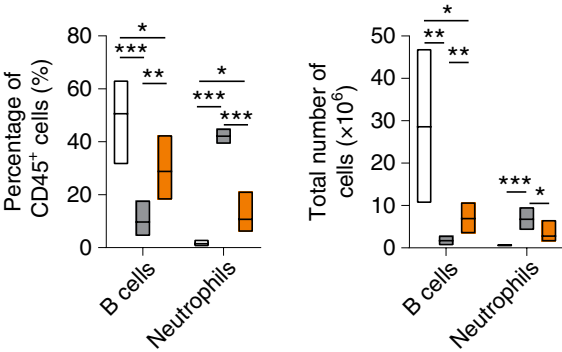

c

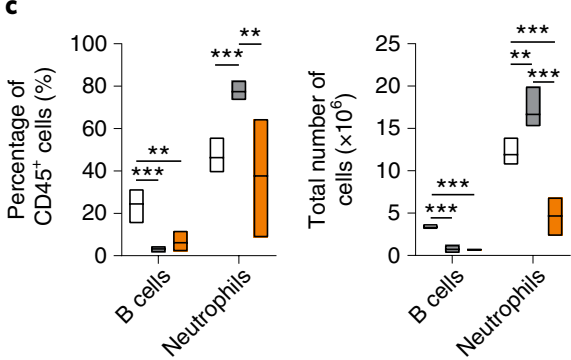

d

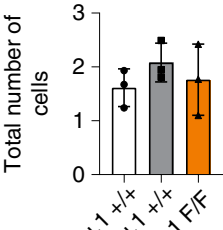

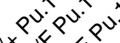

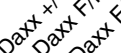
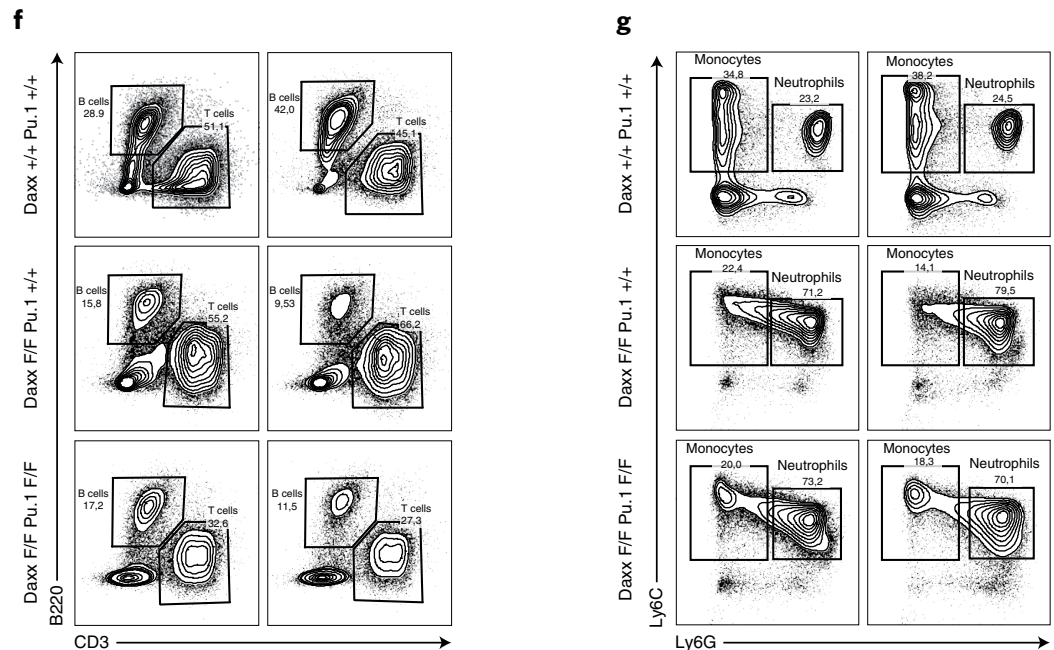

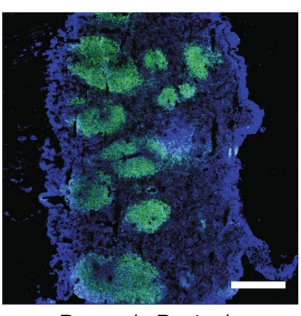

Daxx +/+ Pu. 1 +/+

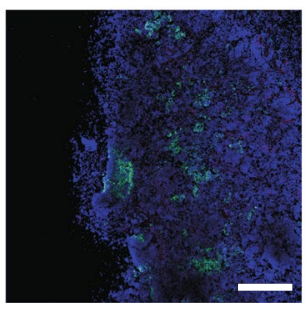

Daxx F/F Pu.1 +/+

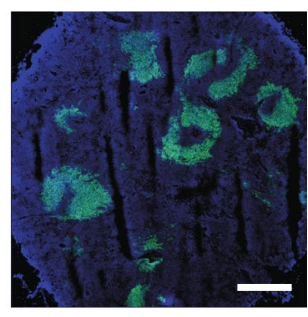

Daxx F/F Pu.1 F/F

i

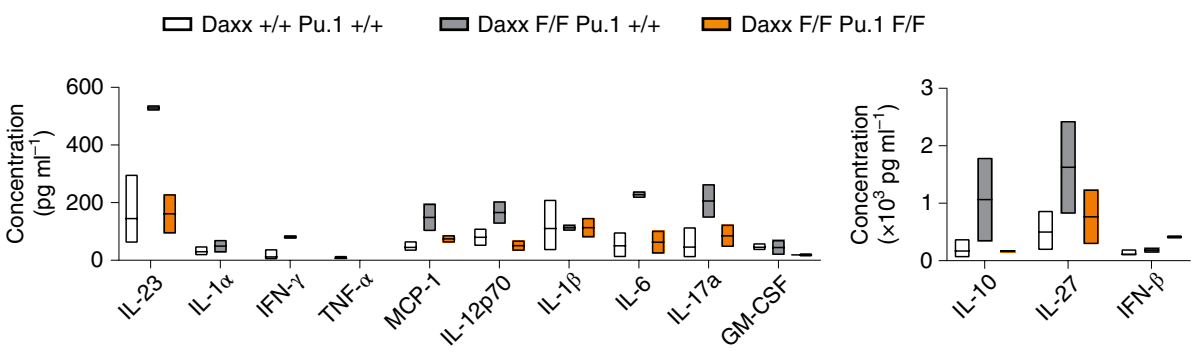

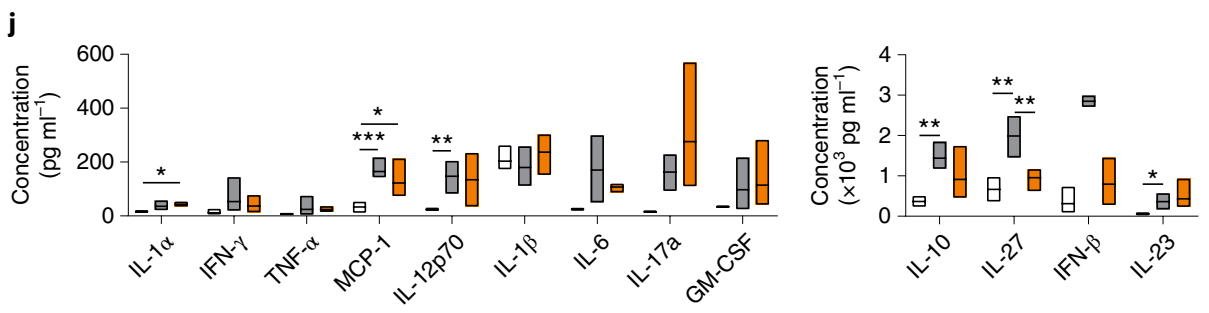

k

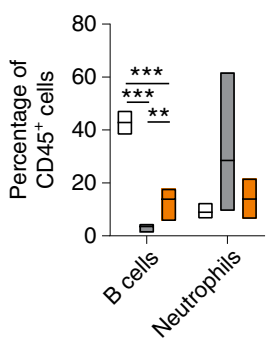

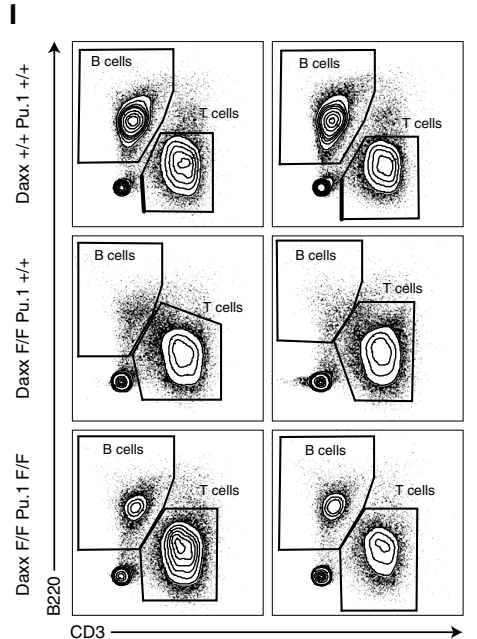


a

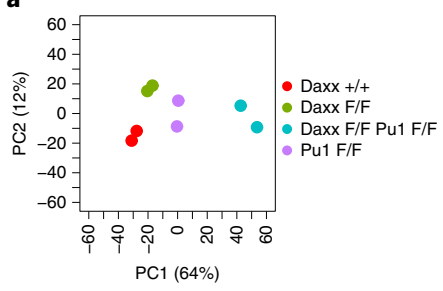

b

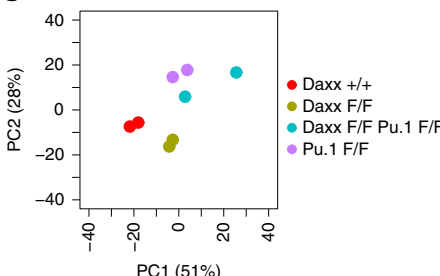

e

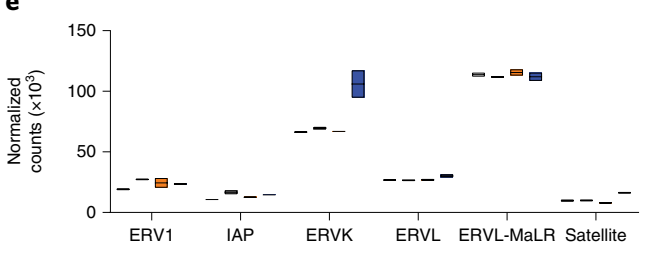

g

- Decreased accessibility Daxx $+/+$

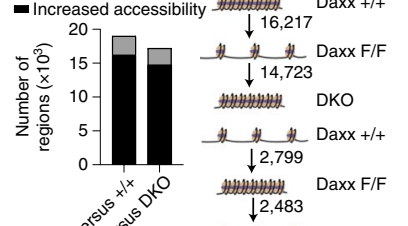

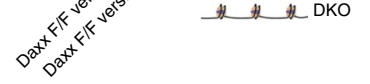

h
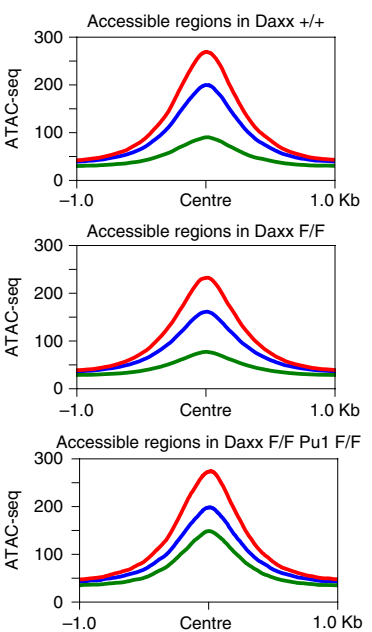

— Daxx +/+ Daxx F/F — Daxx F/F Pu.1 F/F

i

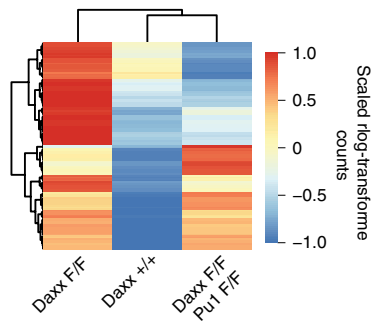

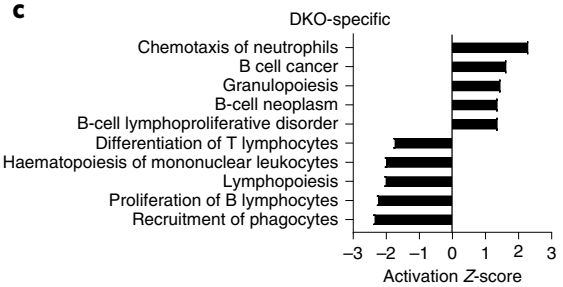

d

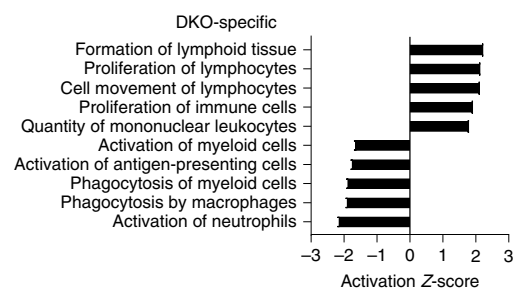

f

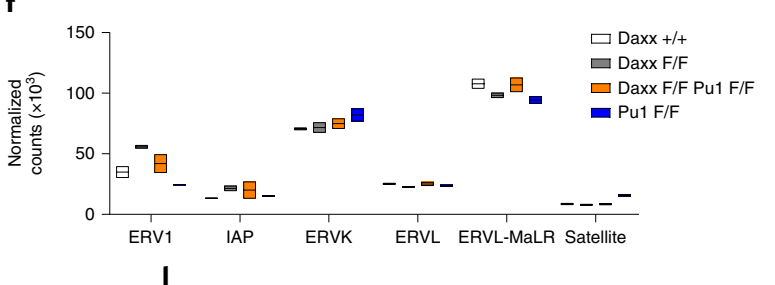

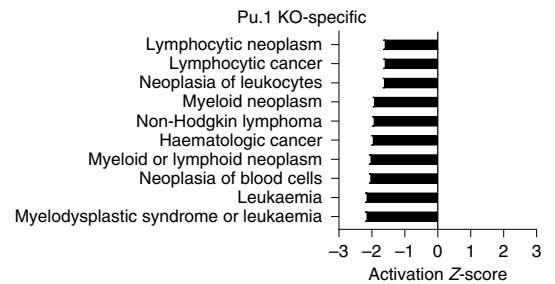

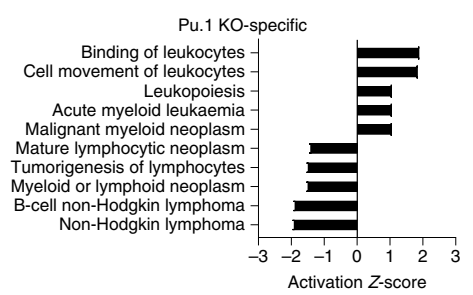

Daxx F/F Pu1 F/F $\square$ Daxx F/F Pu F/F

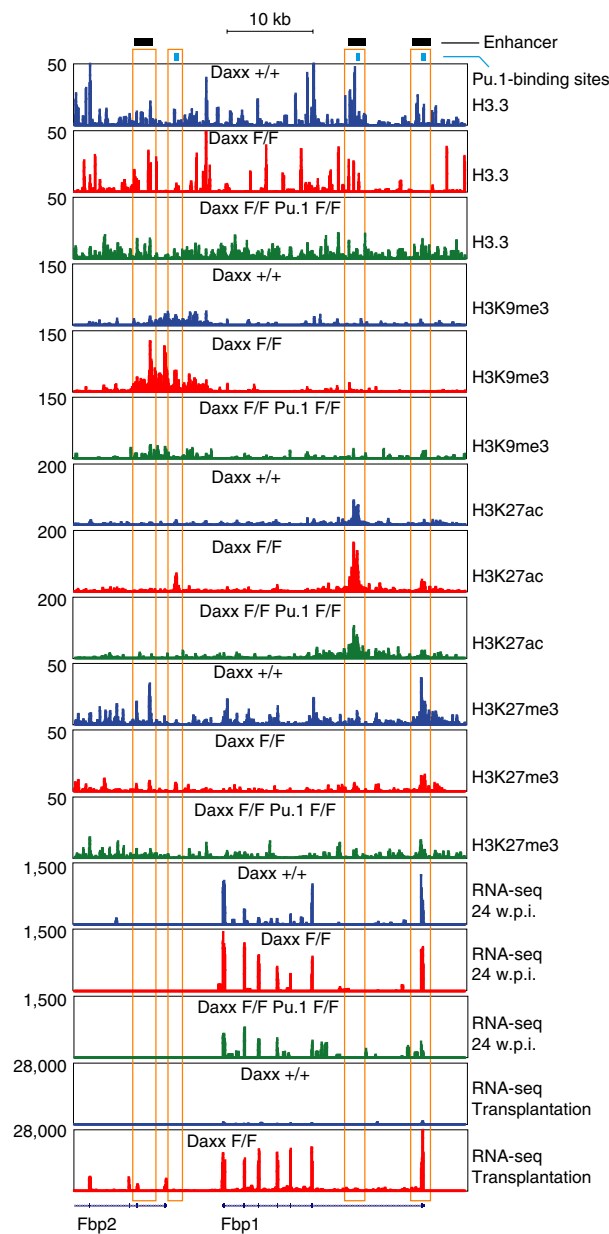

j

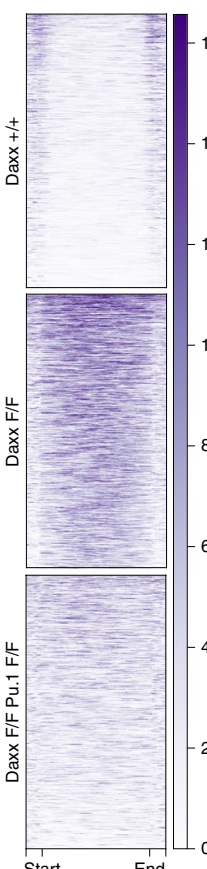

$1 6 \longdiv { 7 } + 1 7 . 5$

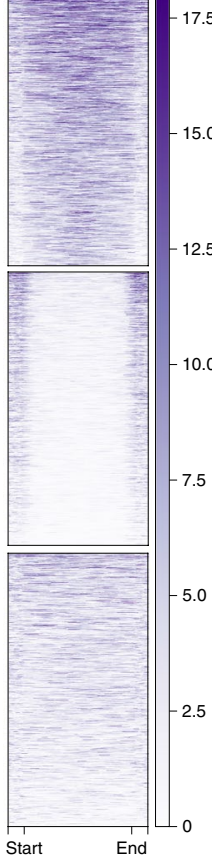

k
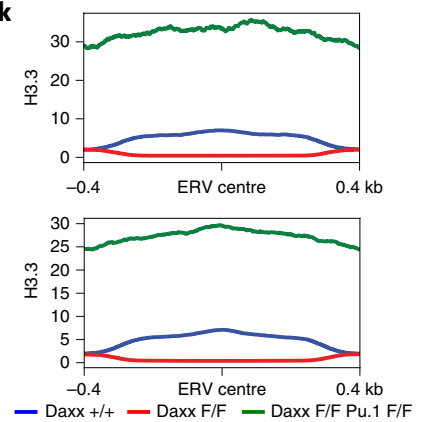
Fig. 8 | Partial rescue of the biological phenotype is associated with specific perturbations at the transcriptional and chromatin levels. a, PCA of the top500 most variable genes in KLS cells collected at 3 w.p.i. b. PCA of the top- 500 most variable genes in GMP cells collected at 3 w.p.i. c, Top-five activated or inhibited haematological functions and diseases associated with KLS cells with Daxx and Pu.1 DKO- (left) and Pu.1-KO-specific (right) gene expression changes. d, Top-five activated or inhibited haematological functions and diseases associated with GMP cells with DKO- (left) and Pu.1-KO-specific (right) gene expression changes. $\mathbf{c}, \mathbf{d}$, Data are the activation Z-score from IPA Fisher's exact tests with multiple testing-adjusted $P<0.05$. An activation $Z$-score $>2$ suggests increased activation of the indicated biofunctions and an activation $Z$-score $<-2$ suggests increased inhibition. $\mathbf{e}$, Normalized read counts of ERV/RTE subtypes and satellite repeats ( $n=2$ mice per genotype) in KLS cells. f, Normalized read counts of ERV/RTE subtypes and satellite repeats ( $n=2$ mice per genotype) in GMP cells. e,f, Boxplots show the minimum and maximum values (box boundaries) and the mean (horizontal line). g, Number of regions that were opened or closed in Daxx-KO versus WT (left) and the number of those regions that reverted back to WT condition in DKO KLS cells (right). h, ATAC-seq read distribution around the centre of IDR-reproducible peaks identified in WT (top), Daxx-KO (middle) and DKO (bottom) KLS cells. i, Heatmap of scaled normalized RNA-seq read counts for genes upregulated in Daxx single-KO KLS and increased accessibility in nearby enhancers. $\mathbf{j}$, Heatmaps of $\mathrm{H} 3 \mathrm{~K} 9 \mathrm{me} 3$ enrichment over distal regions segregated in regions gaining or losing $\mathrm{H} 3 \mathrm{~K} 9 \mathrm{me} 3 . \mathbf{k}, \mathrm{H} 3.3 \mathrm{enrichment} \mathrm{plots} \mathrm{at}$ enhancers (top) and ERVs (bottom). I, Genome browser coverage plot of the Fbp1 and Fbp2 locus. Daxx F/F, Daxx KO and Daxx +/+, Daxx WT; Pu.1 F/F, Pu.1-KO and Pu. $1+/+$, Pu.1 WT. Numerical source data are provided.

transformation during ageing ${ }^{59-62}$. While transposition-driven mutagenesis could lead to the accumulation of potentially leukaemogenic mutations in HSCs and promote clonal haematopoiesis $^{63}$, the resulting anti-viral response may suppress the expansion of mutated $\mathrm{HSCs}^{64}$. In this respect, neutrophilia and inflammation triggered by Daxx loss might be tumour-suppressive. Further genetic alterations (de novo or pre-existing) such as downregulation of Pu.1 may allow Daxx-deficient myeloid or lymphoid progenitors to escape differentiation and undergo neoplastic transformation, in accordance with the reported leukaemogenic effect of Pu.1 downregulation $^{65}$. It is therefore possible that $\mathrm{DKO}$ mice may become leukaemic at old age.

Overall, this work implicates mechanisms governing accessibility to repeat elements in homeostasis of the haematopoietic system via cross-talk with pioneer transcription factors such as Pu.1. Perturbations of these mechanisms may contribute to inflammageing and predispose to leukaemogenesis.

\section{Online content}

Any methods, additional references, Nature Research reporting summaries, source data, extended data, supplementary information, acknowledgements, peer review information; details of author contributions and competing interests; and statements of data and code availability are available at https://doi.org/10.1038/ s41556-021-00774-y.

Received: 3 April 2020; Accepted: 14 September 2021; Published online: 07 December 2021

\section{References}

1. Kunarso, G. et al. Transposable elements have rewired the core regulatory network of human embryonic stem cells. Nat. Genet. 42, 631-634 (2010).

2. Chuong, E. B., Rumi, M. A., Soares, M. J. \& Baker, J. C. Endogenous retroviruses function as species-specific enhancer elements in the placenta. Nat. Genet. 45, 325-329 (2013).

3. Mita, P. \& Boeke, J. D. How retrotransposons shape genome regulation. Curr. Opin. Genet. Dev. 37, 90-100 (2016).

4. Volkman, H. E. \& Stetson, D. B. The enemy within: endogenous retroelements and autoimmune disease. Nat. Immunol. 15, 415-422 (2014).

5. Tugnet, N., Rylance, P., Roden, D., Trela, M. \& Nelson, P. Human endogenous retroviruses (HERVs) and autoimmune rheumatic disease: is there a link? Open Rheumatol. J. 7, 13-21 (2013).

6. Gonzalez-Cao, M. et al. Human endogenous retroviruses and cancer. Cancer Biol. Med. 13, 483-488 (2016).

7. Downey, R. F. et al. Human endogenous retrovirus $\mathrm{K}$ and cancer: innocent bystander or tumorigenic accomplice? Int. J. Cancer 137, 1249-1257 (2015).

8. Manghera, M., Ferguson-Parry, J., Lin, R. \& Douville, R. N. NF- $\kappa$ B and IRF1 induce endogenous retrovirus $\mathrm{K}$ expression via interferon-stimulated response elements in its $5^{\prime}$ long terminal repeat. J. Virol. 90, 9338-9349 (2016).

9. Groh, S. \& Schotta, G. Silencing of endogenous retroviruses by heterochromatin. Cell. Mol. Life Sci. 74, 2055-2065 (2017).
10. He, Q. et al. The Daxx/Atrx complex protects tandem repetitive elements during DNA hypomethylation by promoting H3K9 trimethylation. Cell Stem Cell 17, 273-286 (2015)

11. Elsasser, S. J., Noh, K. M., Diaz, N., Allis, C. D. \& Banaszynski, L. A. Histone H3.3 is required for endogenous retroviral element silencing in embryonic stem cells. Nature 522, 240-244 (2015).

12. Qadeer, Z. A. et al. ATRX in-frame fusion neuroblastoma is sensitive to EZH2 inhibition via modulation of neuronal gene signatures. Cancer Cell 36, 512-527 (2019)

13. Lewis, P. W., Elsaesser, S. J., Noh, K. M., Stadler, S. C. \& Allis, C. D. Daxx is an H3.3-specific histone chaperone and cooperates with ATRX in replication-independent chromatin assembly at telomeres. Proc. Natl Acad. Sci. USA 107, 14075-14080 (2010).

14. Goldberg, A. D. et al. Distinct factors control histone variant H3.3 localization at specific genomic regions. Cell 140, 678-691 (2010).

15. Dyer, M. A., Qadeer, Z. A., Valle-Garcia, D. \& Bernstein, E. ATRX and DAXX: mechanisms and mutations. Cold Spring Harb. Perspect. Med. https://doi.org/10.1101/cshperspect.a026567 (2017).

16. Drane, P., Ouararhni, K., Depaux, A., Shuaib, M. \& Hamiche, A. The death-associated protein DAXX is a novel histone chaperone involved in the replication-independent deposition of H3.3. Genes Dev. 24, 1253-1265 (2010).

17. Ray-Gallet, D. et al. Functional activity of the H3.3 histone chaperone complex HIRA requires trimerization of the HIRA subunit. Nat. Commun. 9 3103 (2018).

18. Lara-Astiaso, D. et al. Immunogenetics. Chromatin state dynamics during blood formation. Science 345, 943-949 (2014).

19. Pundhir, S. et al. Enhancer and transcription factor dynamics during myeloid differentiation reveal an early differentiation block in Cebpa null progenitors. Cell Rep. 23, 2744-2757 (2018).

20. Nguyen, D. T. et al. The chromatin remodelling factor ATRX suppresses R-loops in transcribed telomeric repeats. EMBO Rep. 18, 914-928 (2017).

21. Redon, S., Reichenbach, P. \& Lingner, J. The non-coding RNA TERRA is a natural ligand and direct inhibitor of human telomerase. Nucleic Acids Res. 38, 5797-5806 (2010).

22. Pfeiffer, V. \& Lingner, J. TERRA promotes telomere shortening through exonuclease 1-mediated resection of chromosome ends. PLoS Genet. 8 , e1002747 (2012)

23. Chu, H. P. et al. TERRA RNA antagonizes ATRX and protects telomeres. Cell 170, 86-101 (2017).

24. Zhang, L. F. et al. Telomeric RNAs mark sex chromosomes in stem cells. Genetics 182, 685-698 (2009).

25. Lee, H. R. et al. ERDR1 enhances human NK cell cytotoxicity through an actin-regulated degranulation-dependent pathway. Cell. Immunol. 292, 78-84 (2014).

26. Houh, Y. K., Kim, K. E., Park, H. J. \& Cho, D. Roles of erythroid differentiation regulator 1 (Erdr1) on inflammatory skin diseases. Int. J. Mol. Sci. https://doi.org/10.3390/ijms17122059 (2016).

27. Deneault, E. et al. A functional screen to identify novel effectors of hematopoietic stem cell activity. Cell 137, 369-379 (2009).

28. Michaelson, J. S., Bader, D., Kuo, F., Kozak, C. \& Leder, P. Loss of Daxx, a promiscuously interacting protein, results in extensive apoptosis in early mouse development. Genes Dev. 13, 1918-1923 (1999).

29. Velasco-Hernandez, T., Sawen, P., Bryder, D. \& Cammenga, J. Potential pitfalls of the Mx1-Cre system: implications for experimental modeling of normal and malignant hematopoiesis. Stem Cell Rep. 7, 11-18 (2016).

30. Marzano, A. V. et al. Mechanisms of inflammation in neutrophil-mediated skin diseases. Front Immunol. 10, 1059 (2019). 
31. Mutua, V. \& Gershwin, L. J. A review of neutrophil extracellular traps (NETs) in disease: potential anti-NETs therapeutics. Clin. Rev. Allergy Immunol. https://doi.org/10.1007/s12016-020-08804-7 (2020).

32. Castanheira, F. V. S. \& Kubes, P. Neutrophils and NETs in modulating acute and chronic inflammation. Blood 133, 2178-2185 (2019).

33. Kaplan, M. J. \& Radic, M. Neutrophil extracellular traps: double-edged swords of innate immunity. J. Immunol. 189, 2689-2695 (2012).

34. Regan-Komito, D. et al. GM-CSF drives dysregulated hematopoietic stem cell activity and pathogenic extramedullary myelopoiesis in experimental spondyloarthritis. Nat. Commun. 11, 155 (2020).

35. Hamilton, J. A. Colony-stimulating factors in inflammation and autoimmunity. Nat. Rev. Immunol. 8, 533-544 (2008).

36. Manz, M. G. \& Boettcher, S. Emergency granulopoiesis. Nat. Rev. Immunol. 14, 302-314 (2014).

37. Wollina, U. \& Haroske, G. Pyoderma gangraenosum. Curr. Opin. Rheumatol. 23, 50-56 (2011)

38. Nye, J., Melters, D. P. \& Dalal, Y. The art of war: harnessing the epigenome against cancer. F1000Res. 7, 141 (2018).

39. Chen, C. et al. HIRA, a DiGeorge syndrome candidate gene, confers proper chromatin accessibility on HSCs and supports all stages of hematopoiesis. Cell Rep. 30, 2136-2149 (2020).

40. Hock, H. \& Orkin, S. H. Zinc-finger transcription factor Gfi-1: versatile regulator of lymphocytes, neutrophils and hematopoietic stem cells. Curr. Opin. Hematol. 13, 1-6 (2006).

41. Pang, S. H. et al. PU.1 cooperates with IRF4 and IRF8 to suppress pre-B-cell leukemia. Leukemia 30, 1375-1387 (2016).

42. Iwasaki, H. et al. Distinctive and indispensable roles of PU.1 in maintenance of hematopoietic stem cells and their differentiation. Blood 106, 1590-1600 (2005).

43. Koide, S. et al. Setdb1 maintains hematopoietic stem and progenitor cells by restricting the ectopic activation of nonhematopoietic genes. Blood 128, 638-649 (2016).

44. Lefkopoulos, S. et al. Repetitive elements trigger RIG-I-like receptor signaling that regulates the emergence of hematopoietic stem and progenitor cells. Immunity 53, 934-951 (2020).

45. Pietras, E. M. et al. Re-entry into quiescence protects hematopoietic stem cells from the killing effect of chronic exposure to type I interferons. J. Exp. Med. 211, 245-262 (2014).

46. Sato, T. et al. Interferon regulatory factor-2 protects quiescent hematopoietic stem cells from type I interferon-dependent exhaustion. Nat. Med. 15 696-700 (2009).

47. Hertzog, P. J., O’Neill, L. A. \& Hamilton, J. A. The interferon in TLR signaling: more than just antiviral. Trends Immunol. 24, 534-539 (2003).

48. Dixit, E. et al. Peroxisomes are signaling platforms for antiviral innate immunity. Cell 141, 668-681 (2010).

49. Ashley, C. L., Abendroth, A., McSharry, B. P. \& Slobedman, B. Interferonindependent innate responses to cytomegalovirus. Front Immunol. 10, 2751 (2019)

50. Ashley, C. L., Abendroth, A., McSharry, B. P. \& Slobedman, B Interferon-independent upregulation of interferon-stimulated genes during human cytomegalovirus infection is dependent on IRF3 expression. Viruses https://doi.org/10.3390/v11030246 (2019).

51. Djeghloul, D. et al. Age-associated decrease of the histone methyltransferase SUV39H1 in HSC perturbs heterochromatin and B lymphoid differentiation. Stem Cell Rep. 6, 970-984 (2016).
52. Pasquarella, A. et al. Retrotransposon derepression leads to activation of the unfolded protein response and apoptosis in pro-B cells. Development 143, 1788-1799 (2016).

53. Collins, P. L., Kyle, K. E., Egawa, T., Shinkai, Y. \& Oltz, E. M. The histone methyltransferase SETDB1 represses endogenous and exogenous retroviruses in B lymphocytes. Proc. Natl Acad. Sci. USA 112 8367-8372 (2015).

54. Peters, A. H. et al. Loss of the Suv39h histone methyltransferases impairs mammalian heterochromatin and genome stability. Cell 107, 323-337 (2001)

55. Hoelper, D., Huang, H., Jain, A. Y., Patel, D. J. \& Lewis, P. W. Structural and mechanistic insights into ATRX-dependent and -independent functions of the histone chaperone DAXX. Nat. Commun. 8, 1193 (2017).

56. Hirche, C. et al. Systemic virus infections differentially modulate cell cycle state and functionality of long-term hematopoietic stem cells in vivo. Cell Rep. 19, 2345-2356 (2017).

57. Beerman, I. et al. Functionally distinct hematopoietic stem cells modulate hematopoietic lineage potential during aging by a mechanism of clonal expansion. Proc. Natl Acad. Sci. USA 107, 5465-5470 (2010).

58. Carotta, S., Wu, L. \& Nutt, S. L. Surprising new roles for PU.1 in the adaptive immune response. Immunological Rev. 238, 63-75 (2010).

59. Woods, B. A. \& Levine, R. L. The role of mutations in epigenetic regulators in myeloid malignancies. Immunological Rev. 263, 22-35 (2015).

60. Kramer, A. \& Challen, G. A. The epigenetic basis of hematopoietic stem cell aging. Semin. Hematol. 54, 19-24 (2017).

61. Hu, D. \& Shilatifard, A. Epigenetics of hematopoiesis and hematological malignancies. Genes Dev. 30, 2021-2041 (2016).

62. Herquel, B. et al. Trim24-repressed VL30 retrotransposons regulate gene expression by producing noncoding RNA. Nat. Struct. Mol. Biol. 20, 339-346 (2013)

63. Biechonski, S., Yassin, M. \& Milyavsky, M. DNA-damage response in hematopoietic stem cells: an evolutionary trade-off between blood regeneration and leukemia suppression. Carcinogenesis 38, 367-377 (2017).

64. Barbieri, D. et al. Thrombopoietin protects hematopoietic stem cells from retrotransposon-mediated damage by promoting an antiviral response. J. Exp. Med. 215, 1463-1480 (2018).

65. Basova, P. et al. Aggressive acute myeloid leukemia in PU.1/p53 double-mutant mice. Oncogene 33, 4735-4745 (2014).

Publisher's note Springer Nature remains neutral with regard to jurisdictional claims in published maps and institutional affiliations.

Open Access This article is licensed under a Creative Commons Attribution 4.0 International License, which permits use, sharing, adaptation, distribution and reproduction in any medium or format, as long as you give appropriate credit to the original author(s) and the source, provide a link to the Creative Commons license, and indicate if changes were made. The images or other third party material in this article are included in the article's Creative Commons license, unless indicated otherwise in a credit line to the material. If material is not included in the article's Creative Commons license and your intended use is not permitted by statutory regulation or exceeds the permitted use, you will need to obtain permission directly from the copyright holder. To view a copy of this license, visit http://creativecommons.org/licenses/by/4.0/.

(c) The Author(s) 2021, corrected publication 2022 


\section{Methods}

Patient sample information. We included H\&E staining of skin from patients with pyoderma gangrenosum in our study, which were performed by J.W. at the University of Bonn. The histological picture of the pyoderma gangrenosum lesions was taken from a skin sample of a patient with pyoderma gangrenosum during a normal diagnostic procedure. This routine procedure includes the preparation of H\&E samples. Based on German law, it is possible to take pictures from these skin samples (the patient identity is unknown and no additional investigations were performed with these samples). As a result, a 'patient consent' is not needed. However, the patient did give informed consent to perform the skin biopsy during the diagnostic procedure. This is also in accordance with the Helsinki Ethical guidelines. J.W. has ethical approval from the University of Bonn, which in principle allows the use of skin material taken for diagnostic proposes in later research (BN090/04)

Experimental model details. Mouse models and treatments. The C57BL/6N Daxx conditional-KO $\left(\operatorname{Daxx} \mathrm{F}^{\mathrm{F} / \mathrm{F}}\right)$ mice were generated by Taconic Artemis. The targeting vector contained a neomycin $(\mathrm{NeoR})$ gene surrounded by flipase sequences $(F R T)$ and a puromycin (PuroR) gene surrounded by F3 sites, which were removed by in vivo Flp-mediated recombination. Daxx exons 2-7 were flanked by LoxP sites. C57BL/6N Daxx mice were crossed with C57BL/6J Csf1rCre (JAX, 029206), C57BL/6J Mx1Cre (JAX, 003556) or C57BL/6J Rosa26CreERT2 (JAX, $008463)$ mice. The $\mathrm{C} 57 \mathrm{BL} / 6 \mathrm{~N}$ Hira conditional-KO $\left(\mathrm{Hira}^{\mathrm{F} / \mathrm{F}}\right)$ mice were provided by P. Adams ${ }^{66}$ and crossed with C57BL/6J Mx1Cre mice. The C57BL/6J Pu.1 conditional-KO $\left(P u .1^{\mathrm{F} / \mathrm{F}}\right)$ mice were provided by E. Mass (JAX, 006922) and crossed with C57BL/6 Daxx ${ }^{\mathrm{F} / \mathrm{F}} ; M \times 1 C r e$ mice. C57BL/6J CD45.1 mice were used (JAX, 002014) for the BM chimaera experiments. Genotyping of the C57BL/6N Daxx mice was performed using an Extract-N-amp tissue PCR kit (Sigma-Aldrich) with the primers DAXX_33 (5'-AGATCCTGTCTCTCCTGTCTATCC- $\left.3^{\prime}\right)$ and DA XX 34 (5'-CACTGGGTAGACTAGACTGTGGC-3'). To check for recombination induced by Cre recombinase, we used the primers DAXX_29 (5'-GGAGGGAG TCGAAGAGTTGG-3'), DAXX_30 (5'-TGCGTTTCCTGTCTTTCGG-3') and DAXX_recombined (5'-GCTCACGCCTTTAGTCCGAA-3'). C57BL/6N Hira mice were genotyped using the primers 2292_27 (5'-AATGGTGCTTG CTTTTGTGG-3') and 2292_28 (5'-CCTGCTACCTTATTCTCCAGTCC $\left.-3^{\prime}\right)$, and the primer 2293_30 (5'-GCATTACTTAATCCCCAGATGC-3') was added for the analysis of recombination efficiency. Genotyping of the C57BL/6 Pu.1 mice was performed with the primers PU1_Flox_FW (5'-CTTCACTGCCCATTCATTGGCTCATCA-3') and PU1_Flox_Rev (5'-GCTGGGGACAAGGTTTGATAAGGGAA-3'), and the primer PU1_MUT (5'-CAACCGGATCTAGACTCGAGGA- $3^{\prime}$ ) was added to determine the recombination efficiency. Cre mice were genotyped according to protocols released by The Jackson Laboratory. The mice were housed under specific pathogen-free conditions at the central animal facility of the University College London and German Center for Neurodegenerative Diseases. The mice were bred and subjected to listed procedures according to protocols approved by the English (Home Office) and German authorities (Landesamt für Natur, Umwelt und Verbraucherschutz Nordrhein-Westfalen). Activation of Cre in Daxx;RosaCre $e^{\mathrm{ERT} 2}$ mice was induced by administration of $80 \mathrm{mg} \mathrm{kg}^{-1}$ tamoxifen in corn oil (Sigma-Aldrich) via oral gavage on five consecutive days (ATAC-seq was performed at 3 w.p.i.) or by administration of $100 \mathrm{mg} \mathrm{kg}^{-1}$ tamoxifen in corn oil via intraperitoneal injection (five consecutive days, break of two days, two consecutive days; genomic and phenotypic analyses were performed at 3 d.p.i. and 2 w.p.i.). For the induction of Cre activation in Daxx-KO, Hira-KO and Daxx and Pu. 1 DKO, Mx1Cre mice received three intraperitoneal injections of $300 \mu \mathrm{g} \mathrm{pI}$ :pC (Sigma-Aldrich) administered every other day. The mice were usually treated at 5-11 weeks of age. Mice of both sexes were used in this study. The $n$ value in the figure legends reflects the number of mice analysed in each experiment.

BM chimaera mice. For BM transplantation studies, C57BL/6J CD45.1 mice received a lethal irradiation dose of 10 Gray before transplantation of $2.5 \times 10^{6}$ CD45.2 Daxx ${ }^{+/+} ; M x 1 C r e$ or Daxx ${ }^{\mathrm{F} / \mathrm{F}} ;$ Mx1Cre BM cells together with $1 \times 10^{5}$ CD45.1 $\mathrm{BM}$ support cells. Peripheral blood samples were isolated from the tail vein $4-5$ (first time point) and 8-12 weeks (second time point) post transplantation. The mice were euthanized by $\mathrm{CO}_{2}$ exposure 12-17 weeks post transplantation and analysed as described earlier. Populations of KLS and GMP cells were sorted from recipient BM as described in the 'RNA-seq' section.

Method details. Haematopoietic-cell and organ preparation. Peripheral blood samples were collected from the tail vein or by heart puncture. For the isolation of haematopoietic organs, the mice were euthanized by $\mathrm{CO}_{2}$ exposure. Unless otherwise indicated, the mice were killed two months post treatment. Bone marrow was isolated from the femur and tibia of the hind legs. All isolated organs were directly processed for analysis, frozen for cryopreservation or fixed in neutral buffered formalin (Sigma-Aldrich) for subsequent paraffin embedding. HPCs were isolated from BM using an EasySep mouse hematopoietic progenitor cell isolation kit (STEMCELL Technologies) according to the manufacturer's instructions. If required, RBCs were lysed for $10 \mathrm{~min}$ in ammonium chloride (STEMCELL Technologies) and washed twice before downstream analysis.
Analysis of BM cellularity. Mice were killed two months after induction, except when indicated otherwise. BM cells were isolated from all four hind-leg bones and collected in equal volumes of PBS. The number of cells was determined using a TC20 automated cell counter (Bio-Rad). The RBCs were subsequently lysed for $10 \mathrm{~min}$ in ammonium chloride (STEMCELL Technologies), washed twice and collected in equal volumes of PBS. The number of cells in the samples was calculated again following after RBC lysis. If indicated, analyses were performed separately for male and female mice to control for differences in body size and weight.

Flow cytometry and cell sorting. For flow cytometry analysis, cells were isolated from the specified organs. Dead cells were stained by propidium iodide (Sigma-Aldrich) or Aqua Zombie fixable viability dye (BioLegend) according to the manufacturer's protocol. Fluorochrome-labelled antibodies, as indicated in Supplementary Table 6, were added to the cell suspension for $30 \mathrm{~min}$ on ice. The CD16 and CD32 receptors were blocked with CD16 and CD32 antibodies unless the cells were specifically stained for CD16/32. Intracellular staining was performed according to the manual of the Intracellular fixation and permeabilization buffer set (eBioscience). Lin $^{-}$antibody panels contained antibodies to B220, Gr-1, CD11b, TER-119 and CD3e. For ATAC-seq, the Lin antibody panel included antibodies to B220, CD19, Gr-1, CD11b, CD11c, NK1.1, TER-119 and CD3e. For RNA-seq, the $\mathrm{Lin}^{-}$antibody panel included antibodies to B220, Gr-1, CD11b, TER-119, CD8, CD4 and CD3e. For staining with multiple brilliant violet dyes, the BD Horizon brilliant stain buffer was used according to the manufacturer's instructions. For the assessment of apoptotic B cells, cells isolated from the spleen were first stained with antibodies to CD45, CD11b and B220, followed by staining with an CellEvent caspase-3/7 green flow cytometry assay kit (Invitrogen) according to the manufacturer's instructions. The cells were washed and filtered through a $70 \mu \mathrm{m}$ filter before flow cytometric analysis using a BD LSRFortessa, BD FACSymphony, BD FACSCelesta, BD FACSAriaIII or Beckman Coulter Gallios machine. Cell sorting was performed on a BD FACSAriaIII or BD FACSAria Fusion machine using a $70 \mu \mathrm{m}$ or $100 \mu \mathrm{m}$ nozzle. Data were analysed using the FlowJo software. Neutrophils were gated as CD11b ${ }^{+} \mathrm{Ly} 6 \mathrm{G}^{+} \mathrm{Ly} 6 \mathrm{C}^{\mathrm{intm}} \mathrm{Gr}-1^{\text {hi }}$ and eosinophils as CD11b ${ }^{+}$Ly6 $\mathrm{C}^{\text {intm }} \mathrm{SSC}^{\text {hi }} \mathrm{Ly} 6 \mathrm{G}^{-} \mathrm{Gr}-1^{+}$.

CFU assay with re-plating. For the CFU assays, HPCs were isolated from BM as described in the 'Haematopoietic-cell and organ preparation' section. The HPCs were plated (15,000 cells per $35 \mathrm{~mm}$ dish) in MethoCult M3231 medium supplemented with $100 \mathrm{ng} \mathrm{ml}^{-1} \mathrm{SCF}, 10 \mathrm{ng} \mathrm{ml}^{-1} \mathrm{GM}-\mathrm{CSF}, 10 \mathrm{ng} \mathrm{ml}^{-1} \mathrm{IL}-3$ and $10 \mathrm{ng} \mathrm{ml}^{-1}$ IL-6 (all STEMCELL Technologies) to promote myeloid-cell formation. The cells were cultivated for 1 week at $37^{\circ} \mathrm{C}$. Next, the colonies were counted under a microscope and 15,000 cells were re-plated under the same conditions. After another week of cultivation at $37^{\circ} \mathrm{C}$, the colonies were again counted under a microscope.

For the CFU assays with LT-HSCs, Lin $^{-}$cells were enriched using a MojoSort mouse hematopoietic progenitor cell isolation kit (BioLegend). LT-HSCs were then sorted using a BD FACSAriaIII cell sorter with antibodies to Lin (B220, CD19, Gr-1, CD11b, CD11c, NK1.1, TER-119 and CD3e), Sca-1, c-Kit, CD150 and CD48. The LT-HSCs were collected using the following gating strategy: (1) $\mathrm{c}-\mathrm{Kit}^{+} \mathrm{Lin}^{-}$, (2) $\mathrm{c}-\mathrm{Kit}^{+} \mathrm{Sca}-1^{+}$and (3) $\mathrm{CD} 48^{-} \mathrm{CD} 150^{+}$. To generate myeloid colonies, 400 LT-HSCs were sorted into $270 \mu$ IMDM medium (STEMCELL Technologies) supplemented with $2 \%$ FBS, $1 \%$ Pen-Strep and $1 \%$ L-glutamine, with the addition of the following growth factors: SCF $\left(100 \mathrm{ng} \mathrm{ml}^{-1}\right)$, GM-CSF $\left(10 \mathrm{ng} \mathrm{ml}^{-1}\right), \mathrm{IL}-3$ $\left(10 \mathrm{ng} \mathrm{ml}^{-1}\right)$ and IL-6 $\left(10 \mathrm{ng} \mathrm{ml}^{-1}\right)$. All growth factors and cytokines were purchased from Preprotech. After the addition of $30 \mu$ l Pen-Strep, the cell suspension was added to $3 \mathrm{ml}$ MethoCult M3231 (STEMCELL Technologies) and $2 \times 1.1 \mathrm{ml}$ was seeded into $35 \mathrm{~mm}$ dishes. The colonies were counted on day 7 . On day 10, the cells were harvested and $3 \times 10^{5}$ cells were re-plated. Residual cells were analysed by flow cytometry. Seven days after re-plating, the new colonies were counted and on day 10 , the cells were harvested and analysed by flow cytometry.

RNA isolation and quantitative PCR with reverse transcription. Total RNA was isolated from BM cells using an RNeasy mini kit (Qiagen) according to the manufacturer's instructions. The RNA concentration was determined using a ND1000 Spectrophotometer (NanoDrop), followed by reverse transcription using a High-capacity cDNA reverse transcription kit (Applied Biosystems). Quantitative real-time PCR was performed on a 7500 Fast real-time PCR system (Applied Biosystems) using the Fast SYBR Green master mix (Applied Biosystems) with the primers mDaxx sense (5'-GATGACTATAGGCCAGGCGT-3'), mDaxx antisense $\left(5^{\prime}\right.$-TCGTCTCTTCTGTCTCTCGC-3'), mHira sense $\left(5^{\prime}\right.$-GTTGT CATTTGGAATGCCGTGA-3'), mHira antisense (5'-CAGCGTCCTCC ATACCTTCA-3'), mTBP sense (5'-AGCTCTGGAATTGTACCGCAG-3') and mTBP antisense ( $5^{\prime}$-GACTGCAGCAAATCGCTTGGG- $\left.3^{\prime}\right)$. The relative abundance of the specific transcripts was normalized to TATA box binding protein (TBP) messenger RNA and calculated using the $2^{-\Delta \Delta c_{\mathrm{t}}}$ method.

Western blotting. Cells were washed in PBS and lysed in RIPA buffer with cOmplete protease inhibitor cocktail (Roche). The lysed samples were sonicated for $10 \mathrm{~s}$ and the protein concentrations were determined using a Pierce protein 
assay kit (Thermo Fisher) according to the manufacturer's instructions. The proteins were separated by SDS-PAGE and blotted on a nitrocellulose membrane. Blocking was performed for $1 \mathrm{~h}$ in $5 \%$ non-fat dry milk (PBS-T) or $4 \%$ BSA (TBS-T) at room temperature and the membranes were incubated overnight with primary antibodies at $4{ }^{\circ} \mathrm{C}$. The primary antibodies were detected by horseradish peroxidase-conjugated secondary antibodies and subsequently visualized using the ChemiDoc XRS+ imaging system (Bio-Rad). The antibodies used are specified in Supplementary Table 12. Normalization of protein levels was performed by densitometry (ImageLab software, Bio-Rad).

Immunofluorescence and $H \& E$ staining. For immunofluorescence imaging, cells were spun down using a Cytospin 4 (Thermo Fisher Scientific) cytocentrifuge at 600 r.p.m. for $5 \mathrm{~min}$. Frozen sections were cut to $7-15 \mu \mathrm{m}$ sections using a CryoStar NX70 (Thermo Fisher Scientific) cryostat. The cells and tissue sections were fixed in $4 \%$ paraformaldehyde for $20 \mathrm{~min}$, washed three times in PBS-T (PBS with $0.1 \%$ Triton X-100) and permeabilized for 15 min using $0.3 \%$ Triton X-100. Blocking was performed for $1 \mathrm{~h}$ in $5 \%$ goat serum and the samples were incubated with primary antibodies overnight at $4{ }^{\circ} \mathrm{C}$. The antibodies are listed in Supplementary Table 12. After washing in PBS-T, the samples were incubated with secondary antibodies for $90 \mathrm{~min}$ at room temperature, washed $3 \times$ in PBS-T and the nuclei were stained for $10 \mathrm{~min}$ in $1 \mu \mathrm{g} \mathrm{ml}^{-1}$ Hoechst 33342 (Thermo Fisher Scientific), followed by additional wash steps. The cells and tissue sections were mounted using Roti-Mount Aqua (ROTH) medium. Images were obtained on a Zeiss LSM700 or LSM800 laser scanning microscope. For the H\&E staining, organs were isolated from mice, fixed in $10 \%$ neutral buffered formalin (Sigma-Aldrich) and subsequently processed for paraffin embedding. Tissue sections were cut to 5-15 $\mu \mathrm{m}$ using a CUT5062 microtome (SLEE medical). The sections were washed twice for $5 \mathrm{~min}$ in xylene, followed by a $5 \mathrm{~min}$ incubation in a 90:10 xylene:ethanol mix. Subsequently, rehydration steps were performed in absolute ethanol $(2 \times)$, $95 \%$ ethanol, $80 \%$ ethanol and $70 \%$ ethanol, $5 \mathrm{~min}$ each, followed by a brief rinse in water. The samples were incubated with haematoxilin (Life Technologies) for $2 \mathrm{~min}$, followed by rinsing in water and $0.25 \%$ ammonia water. The slides were incubated for $1 \mathrm{~min}$ in $95 \%$ ethanol, followed by a 2 min stain in alcoholic eosin Y (Sigma-Aldrich). Dehydration was performed in 95\% ethanol and absolute ethanol ( $3 \times ; 1 \mathrm{~min}$ each), followed by incubation in xylene ( $3 \times ; 1 \mathrm{~min}$ each). The sections were mounted with DPX (Sigma-Aldrich). Images were taken on a Zeiss Epi-Scope microscope.

Cytokine analysis. Blood samples were obtained from mice and spun at $1,000 \mathrm{~g}$ for $10 \mathrm{~min}$ at $4^{\circ} \mathrm{C}$ to collect the plasma. Cytokine concentrations were determined using a LEGENDplex mouse inflammation panel (BioLegend, 740446) and mouse HSC panel (BioLegend, 740677) according to the manufacturer's recommendations. Analysis was performed on a BD FACSymphony machine and using the LEGENDplex data analysis software (BioLegend).

Northern blotting. RNA was isolated from BM cells using a Direct-zol RNA miniprep plus kit (Zymo Research) according to the manufacturer's protocol. Subsequently, DNA was digested using a Turbo DNA-free kit (Thermo Fisher) following the manufacturer's protocol. The RNA was recovered and concentrated using an RNA clean and concentrator-5 kit (Zymo Research). Northern blot analysis was performed according to standard procedures. Briefly, $5 \mu \mathrm{g}$ of total RNA was run on a $1 \%$ denaturing agarose gel. The RNA was transferred to a Hybond-N+ membrane (GE Healthcare) by capillary transfer and fixed by ultraviolet-light crosslinking. The membrane was pre-hybridized at $42^{\circ} \mathrm{C}$ for 20 min and then hybridized in PerfectHyb plus hybridization buffer (Sigma) containing $1 \times 10^{6} \mathrm{cpm} \mathrm{ml}^{-1}$ of ${ }^{32} \mathrm{P}$-labelled TERRA DNA probe $5^{\prime}$-(TAACCC) 5-3 and $0.1 \mathrm{mg} \mathrm{ml}^{-1}$ herring sperm DNA (Thermo Fisher). Hybridization was carried out overnight at $42{ }^{\circ} \mathrm{C}$. The following day, the membrane was washed once in low stringency buffer $(2 \times$ SSC and $0.1 \%$ SDS $)$ at room temperature for $5 \mathrm{~min}$ and twice in high stringency buffer $(0.5 \times$ SSC and $0.1 \%$ SDS $)$ at $42^{\circ} \mathrm{C}$ for $20 \mathrm{~min}$. The membranes were exposed to muliautoradiography film at $-80^{\circ} \mathrm{C}$. After exposure, the membrane was stripped in boiling stripping buffer $(0.1 \%$ SDS and $5 \mathrm{mM}$ EDTA $)$ and re-hybridized using a GAPDH probe (5'-GTAGACCCACGACATACTCAGCACCGGCCTCACCCCATT-3') as a loading control.

RNA-seq of cells isolated from transplanted animals. CD45.2+ $2^{+} \mathrm{KS}$ and GMP $\left(\mathrm{Lin}^{-} \mathrm{C}-\mathrm{Kit}^{+} \mathrm{Sca}-1^{-} \mathrm{CD} 16 / 32^{+} \mathrm{CD} 34^{+}\right.$) populations were sorted from the BM of transplanted mice. Total RNA was isolated from samples containing between 4,700 and 246,000 cells using TRIzol reagent (Invitrogen) and a miRNeasy micro kit (Qiagen) according to the manufacturer's protocol. The precipitated RNA was resuspended in RNase-free water and the RNA quantity and quality (RINe) were assessed via the HS RNA analysis screen tape assay on a 4200 TapeStation system (Agilent Technologies). The total RNA was converted into double-stranded complementary DNA libraries as the template for high-throughput sequencing using the Ovation SoLo RNA-seq kit (NuGEN Technologies). Briefly, the DNA was digested and first-strand DNA was synthesized using random hexamers. After second-strand synthesis, the ends were repaired, followed by adaptor ligation and PCR pre-amplification. NuGEN proprietary AnyDeplete probes were added to selectively block fragments originating from ribosomal RNA from amplification in the subsequent PCR reaction. Size-selection and purification of cDNA fragments of approximately 200-500 bp in length was performed using AMPure XP beads (Beckman Coulter). The size distribution of the cDNA libraries was measured using the HS D1000 DNA assay on a 4200 TapeStation system (Agilent Technologies). The cDNA libraries were quantified using a KAPA library quantification kit (Kapa Biosystems). After cluster generation on a cBot (Illumina), the libraries were sequenced in a paired-end $2 \times 101 \mathrm{bp}$ run on a HiSeq 1500 system (Illumina) using TruSeq v3 chemistry and de-multiplexed using CASAVA version 1.8.4. The experiment was run in duplicate.

RNA-seq of cells collected at steady state. LT-HSCs $\left(\mathrm{Lin}^{-} \mathrm{c}-\mathrm{Kit}^{+} \mathrm{Sca}-1^{+} \mathrm{Il}-\right.$

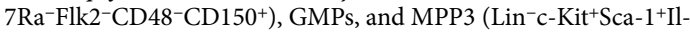

7Ra ${ }^{-}$Flk2 $\left.{ }^{-} \mathrm{CD} 48^{+} \mathrm{CD} 150^{-}\right)$, MPP4 (Lin $\left.{ }^{-} \mathrm{c}-\mathrm{Kit}^{+} \mathrm{Sca}-1^{+} \mathrm{Il}-7 \mathrm{Ra}^{-} \mathrm{Flk2}{ }^{+} \mathrm{CD} 48^{+} \mathrm{CD} 150^{-}\right)$ and KLS cells were sorted from BM 3 d.p.i. (RosaCreER mice: LT-HSCs), 3 w.p.i. (Mx1Cre mice: GMPs and KLS, MPP3 and MPP4 cells) or 24 w.p.i. (Mx1Cre mice: KLS cells and GMPs). Total RNA was isolated from 2,000-5,000 cells using a NucleoSpin RNA XS plus kit (Macherey-Nagel) according to the manufacturer's instructions. The RNA quantity and quality (RINe) were assessed using the HS RNA analysis screen tape assay on a 4200 TapeStation system (Agilent Technologies). Total RNA was converted into double-stranded cDNA libraries as a template for high-throughput sequencing using the SMARTer stranded total RNA-seq kit v.2-pico input mammalian kit (TaKaRa Bio) according to the manufacturer's instructions. Briefly, after first-strand cDNA synthesis with SMARTer oligonucleotides, barcoded adaptors for Illumina sequencing were added via limited-cycle PCR. The PCR products were purified and ribosomal cDNA was then depleted. The remaining cDNA fragments were further amplified by PCR ( 15 cycles) with primers universal to all libraries. After purifying the resulting PCR products, the size distribution of the cDNA libraries was measured using the HS 5000 DNA assay on a 4200 TapeStation system (Agilent Technologies) and the cDNA concentrations were determined using a Qubit dsDNA HS assay kit (Thermo Fisher Scientific). After cluster generation, the samples were sequenced either as single-end $1 \times 100 \mathrm{bp}$ or paired-end $2 \times 100 \mathrm{bp}$ on a NovaSeq 6000 system (Illumina). The experiments were run in duplicate or triplicate.

ATAC-seq of RosaCreER mice at 3 w.p.i. For ATAC-seq, RosaCreER Daxx mice were killed 3 weeks after tamoxifen treatment. The BM was isolated from the femur and tibia, followed by RBC lysis and sorting of CMP ( $\mathrm{Lin}^{-} \mathrm{CD} 45.2^{+} \mathrm{c}-\mathrm{Kit}^{+} \mathrm{Sca}-1^{-}$ $\mathrm{CD} 16 / 32^{-} \mathrm{CD}^{+} 4^{+}$and GMP populations following antibody staining. Enrichment of c-Kit ${ }^{+}$cells using CD117 MicroBeads (Miltenyi Biotec) was performed before sorting LT-HSCs $\left(\mathrm{Lin}^{-} \mathrm{CD} 45.2^{+} \mathrm{c}-\mathrm{Kit}^{+} \mathrm{Sca}-1^{+} \mathrm{CD} 48^{-} \mathrm{CD} 150^{+}\right.$). Between 8,000 and 50,000 flow-sorted cells were collected in flow buffer (PBS, $2 \%$ FBS and $2 \mathrm{mM}$ EDTA) and immediately processed following previously published protocols ${ }^{67}$. Briefly, the sorted cells were spun down at $500 \mathrm{~g}$ for $5 \mathrm{~min}$ at $4{ }^{\circ} \mathrm{C}$, washed once in cold PBS and spun down in $50 \mu \mathrm{l}$ cold lysis buffer $(10 \mathrm{mM}$ Tris- $\mathrm{HCl} \mathrm{pH} \mathrm{7.4,}$ $10 \mathrm{mM} \mathrm{NaCl}, 3 \mathrm{mM} \mathrm{MgCl}_{2}$ and $0.1 \%$ IGEPAL CA-630) at $500 \mathrm{~g}$ for $10 \mathrm{~min}$ at $4{ }^{\circ} \mathrm{C}$. Immediately thereafter the tagmentation reaction was started by adding Nextera's Tn 5 transposase (TDE1) in reaction buffer. The transposition reaction mix was incubated for $30 \mathrm{~min}$ at $37^{\circ} \mathrm{C}$, followed by DNA purification using a MinElute PCR purification kit (Qiagen). ATAC-seq libraries were generated from tagmented DNA by PCR amplification using Nextera index primers (Illumina) according to the manufacturer's protocol. The ATAC-seq libraries were purified using a PCR purification MinElute kit (Qiagen) and quantified using KAPA library quantification kits (Kapa Biosystems) and a D1000 assay on a Tapestation 4200 (Agilent). The libraries were sequenced in a paired-end $2 \times 101 \mathrm{bp}$ run on a HiSeq 1500 system (Illumina) using TruSeq v3 chemistry and de-multiplexed using CASAVA version 1.8.4. In addition, ATAC-seq libraries from CMP and GMP cells were sequenced as a single-read $75 \mathrm{bp}$, rapid run on the HiSeq 1500 system using HiSeq Rapid v.2 chemistry. The resulting data were also de-multiplexed using CASAVA version 1.8.4. The experiment was run in duplicates.

ATAC-seq of 3 d.p.i. LT-HSCs and 3 w.p.i. KLS cells. LT-HSCs ( Lin $^{-}{ }^{-}-\mathrm{Kit}^{+} \mathrm{Sca}-1^{+} \mathrm{Il}-$ 7Ra-Flk2-CD48-CD150+) and KLS cells were sorted from BM 3 d.p.i. (LT-HSCs) or 3 w.p.i. (KLS cells). The LT-HSCs $(2,000)$ and KLS cells $(5,000)$ were directly sorted into $12.5 \mu \mathrm{l}$ of $2 \times \mathrm{TD}$ buffer (Illumina) using the FACSAriaIII. Directly after sorting, $0.25 \mu \mathrm{l}$ of $1 \%$ digitonin (Promega), $0.25 \mu$ l of $10 \%$ Tween-20 (Merck Millipore) and nuclease-free water were added to each sample to obtain a final volume of $23.5 \mu \mathrm{l}$. After mixing the samples by vortexing, $1.5 \mu \mathrm{l}$ of the TDE tagmentation enzyme (Illumina) was added to each sample. The samples were incubated for $30 \mathrm{~min}$ at $37^{\circ} \mathrm{C}$ with shaking at 1,000 r.p.m. to induce tagmentation, followed by DNA purification using a MinElute reaction clean up kit (Qiagen). ATAC-seq libraries were generated from the tagmented DNA by PCR amplification (13 cycles) using the 'IDT for Illumina Nextera DNA UD Indexes Set A' primers (Illumina) and the KAPA HiFi hot start ready mix (Roche). The ATAC-seq libraries were size selected (175-1,300 bp), purified using SPRIselect beads (Beckman Coulter) and quantified using a HS D5000 assay on a Tapestation 4200 (Agilent). 
The libraries were sequenced in a paired-end $2 \times 100$ bp run on a NovaSeq 6000 system (Illumina). The experiments were run in duplicate or triplicate.

CUT\&Tag. HSPCs were isolated by magnetic cell sorting by first enriching Lin $^{-}$cells using an EasySep mouse hematopoietic progenitor cell isolation kit (STEMCELL Technologies) according to the manufacturer's instructions and then isolating $\mathrm{c}-\mathrm{Kit}^{+}$cells from the resulting cell suspension using mouse CD117 MicroBeads (Miltenyi Biotech). The nuclei were isolated, washed and frozen in wash buffer according to the 'Bench top CUT\&Tag V.3' protocol (https:// ww.protocols.io/view/bench-top-cut-amp-tag-bcuhiwt6) ${ }^{68}$. For the actual CUT\&Tag experiment, we followed the CUT\&Tag-direct protocol described by Henikoff et al. ${ }^{69,70}$ using the CUTANA pAG-Tn5 enzyme (Epycypher). Briefly, aliquots of 30,000-45,000 native nuclei per reaction were bound to activated Concanavalin A beads. After successive incubations with primary (overnight at $\left.4^{\circ} \mathrm{C}\right)$ and secondary $(0.5-1 \mathrm{~h})$ antibodies in wash buffer $(20 \mathrm{mM}$ HEPES $\mathrm{pH} 7.5$, $150 \mathrm{mM} \mathrm{NaCl}, 0.5 \mathrm{mM}$ spermidine and $1 \times$ protease inhibitor), the beads were washed and resuspended in pAG-Tn5 (1:20 dilution) in 300-wash buffer (wash buffer containing $300 \mathrm{mM} \mathrm{NaCl}$ ) for $1 \mathrm{~h}$. The incubations were performed at room temperature, except when otherwise stated, in volumes of $25-50 \mu \mathrm{l}$ in low-retention PCR tubes. Tagmentation was performed for $1 \mathrm{~h}$ in 300-wash buffer supplemented with $10 \mathrm{mM} \mathrm{MgCl}_{2}$. Following tagmentation, the beads were washed in $50 \mu \mathrm{l}$ TAPS buffer (10 mM TAPS pH 8.5 and $0.2 \mathrm{mM}$ EDTA), resuspended in $5 \mu \mathrm{l}$ SDS release buffer $(0.1 \%$ SDS and $10 \mathrm{mM}$ TAPS $\mathrm{pH} 8.5)$ and incubated for $1 \mathrm{~h}$ at $58^{\circ} \mathrm{C}$. The SDS was neutralized with $15 \mu \mathrm{l}$ of $0.67 \%$ Triton X-100, and $4 \mu \mathrm{l}$ of dual-indexed primers from the 'IDT for Illumina Nextera DNA UD Indexes Set A' (Illumina) as well as $25 \mu \mathrm{l}$ of NEBNext high-fidelity $2 \times \mathrm{PCR}$ master mix (NEB) were added. Gap filling and 18 cycles of PCR were performed, followed by clean-up with $65 \mu$ of SPRIselect beads (Beckman Coulter). We used antibodies, at a 1:20 dilution, to H3K9me3 (Active Motif), H3K27ac (Epicypher), H3K27me3 (Cell Signaling Technologies), histone H3.3 (Merck Millipore) and Pu.1 (Abcam). The size distribution and concentration of the CUT\&Tag libraries were measured using the HS 5000 DNA assay on a 4200 TapeStation system (Agilent Technologies). After cluster generation, the samples were sequenced either as paired-end $2 \times 75 \mathrm{bp}$ on a NextSeq 500 system (Illumina) or as paired-end $2 \times 50$ bp on a NovaSeq 6000 system (Illumina). The experiments were run in duplicate or triplicate.

Bioinformatics analysis. A detailed description of the bioinformatics analyses for the ATAC-seq, RNA-seq and CUT\&Tag data can be found in Supplementary Note 1 .

Statistics and reproducibility. Statistical analyses were performed using GraphPad Prism (GraphPad Software) or the R program. Summarized views on data that underlie the statistical tests as well as the exact $P$ values are available in the source data. The statistical details for each experiment are also provided in the figure legends. Statistical tests were run as two-sided tests, when appropriate. Most experiments were performed independently at least twice; details are provided in the figure legends. The genomic assay data (Figs. 1,5,6,8 and Extended Data Figs. 1,7-10) are based on two or three biological replicates. The experimental data in Figs. 2g,i,k,p, 3b,f, 4e,f, 7a,d,i and Extended Data Figs. 2d, 4b,c, 8b,d,e are based on biological replicates ( $n$ is given in the figure legends) that were not repeated independently on a different day.

Reporting Summary. Further information on research design is available in the Nature Research Reporting Summary linked to this article.

\section{Data availability}

All sequencing data that support the findings of this study can be found at Gene Expression Omnibus under the accession number GSE119309. An overview of the genomics studies run as part of this study can be found in Supplementary Table 11. Previously published sequencing data that were re-analysed here are available under the accession codes GSE60101 and GSE79180 (SRR2062971 and SRR2062968). The GTF and FASTA files used for Bioinformatics analysis (mm10, GENCODE release M14) can be downloaded from GENCODE (https:// www.gencodegenes.org/mouse/release_M14.html). All other data supporting the findings of this study are available from the corresponding author on reasonable request. Source data are provided with this paper.

\section{Code availability}

Rscripts exemplifying the counting of paired-end (Supplementary Code 1) and single-end RNA-seq reads (Supplementary Code 1) are available online in Supplementary Note 1 . The custom Rscript to generate non-overlapping mouse gene annotations as well as the created gene annotations used for RNA-seq read counting are available from the corresponding author on request.

\section{References}

66. Rai, T. S. et al. HIRA orchestrates a dynamic chromatin landscape in senescence and is required for suppression of neoplasia. Genes Dev. 28, 2712-2725 (2014)

67. Buenrostro, J. D., Giresi, P. G., Zaba, L. C., Chang, H. Y. \& Greenleaf, W. J. Transposition of native chromatin for fast and sensitive epigenomic profiling of open chromatin, DNA-binding proteins and nucleosome position. Nat. Methods 10, 1213-1218 (2013).

68. Kaya-Okur, H. S. et al. CUT\&Tag for efficient epigenomic profiling of small samples and single cells. Nat. Commun. 10, 1930 (2019).

69. Henikoff, S., Henikoff, J. G., Kaya-Okur, H. S. \& Ahmad, K. Efficient chromatin accessibility mapping in situ by nucleosome-tethered tagmentation. eLife https://doi.org/10.7554/eLife.63274 (2020).

70. Shen, L. et al. diffReps: Detecting differential chromatin modification sites from ChIP-seq data with biological replicates. PLoS ONE 8, e65598 (2013).

\section{Acknowledgements}

A special thanks to D. Bano, M. Stork and other members of their team (DZNE). We also thank Steven Zvi Josefowicz (Cornell University), H. de The (College De France/INSERM) and N. Jabado (McGill University) for input and discussion, B. Vanhaesebroeck (UCL) for providing the Rosa26CreERT2 mice, the P.S. lab (in particular, X. Yan, C. Georgopoulou M. Chevallot-Beroux and D.A.) for assistance with experiments and scientific discussion, the DZNE Core Facilities, DZNE animal facility, PRECISE, the LIMES animal facility, UCL Core Services and UCL Biological Services. This work was in part funded by DZNE, the Worldwide Cancer Research Fund (WCRF) and the UCL Biomedical Research Centre. J.P.G. was partly supported by a fellowship from the Deutsche Forschungsgemeinschaft (DFG; German Research Foundation). P.S. was supported by the H3.3Cancer ERC Consolidator award until May 2019 and is currently supported by a project grant on histone variants from the Wilhelm Sander Stiftung. J.L.S., M.B., E.M., M.C. and P.S. are supported by the DFG under Germany's Excellence Strategy (grant no. EXC2151-390873048; Excellence Cluster ImmunoSensation2). M.C. was supported by a Bennett Fellowship from Bloodwise (grant no. 12002) and the Helmholtz-Gemeinschaft, Zukunftsthema Immunology and Inflammation (grant no. ZT-0027). R.N. and J.K. were funded by a Kay Kendall Leukaemia Fund Intermediate Fellowship. J.R. is a recipient of a Marie Sklodowska-Curie Actions Individual Fellowship (2020-2022). R.Z. and P.S. are supported by the Helmholtz-Gemeinschaft Aging and Metabolic Programming (AMPro) Consortium. T.E., P.S. and P.D. received funding from the UK Medical Research Council. C.B. was supported by the Swedish Foundations' Starting Grant (SFSG), StemTherapy, Swedish Research Council and Cancerfonden. C.B. is a Ragnar Söderberg Fellow in Medicine and Cancerfonden Young Investigator. P.D.A. is supported by NIA grant no. P01 AG031862-13. This work was supported by the UCL Cancer Institute Flow Cytometry Facility, which is funded by the CRUK-UCL Centre Award (grant no. C416/A25145) and the CRUK Cancer Immunotherapy Network Accelerator (CITA) Award (grant no. C33499/A20265) as well as CRUK Centre award to Barts Cancer Centre (grant no. C16420/A18066).

\section{Author contributions}

J.P.G., J.R. and P.S. conceived the original idea, experimental design and overall strategy. V.C. contributed to the experimental design. J.P.G., J.R., V.C., N.O., S.S., N.G., N.I., S.M., K.H., P.D., A.H.K., H.-M.L., S.P., T.S., R.Z. and J.K. conducted and analysed experiments. J.P.G, J.R. and P.S. wrote the manuscript. J.R. led the revisions of the manuscript. P.D.A., T.M., T.E., J.W., M.B., C.B., J.L.S., M.C., R.N., E.M. and P.S. acquired funding and provided supervision.

\section{Funding}

Open access funding provided by Deutsches Zentrum für Neurodegenerative Erkrankungen e.V. (DZNE) in der Helmholtz-Gemeinschaft.

\section{Competing interests}

The authors declare no competing interests.

\section{Additional information}

Extended data is available for this paper at https://doi.org/10.1038/s41556-021-00774-y.

Supplementary information The online version contains supplementary material available at https://doi.org/10.1038/s41556-021-00774-y.

Correspondence and requests for materials should be addressed to Paolo Salomoni.

Peer review information Nature Cell Biology thanks Gerald de Haan, Yuin-Han Loh and the other, anonymous, reviewer(s) for their contribution to the peer review of this work. Peer reviewer reports are available.

Reprints and permissions information is available at www.nature.com/reprints. 
a

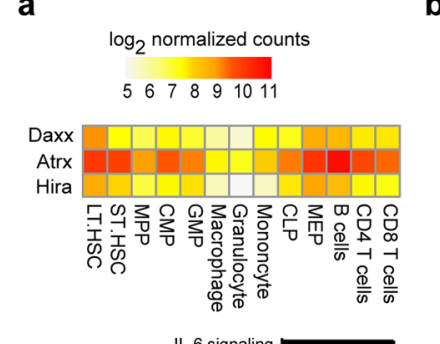

dReceptor-mediated Phagocyltosis

d Receptor-mediated Phagocytosis.
p70-S6K signaling.

2-mediated oxidative stress response

Ephrin receptor signaling

ErbB signaling
Mouse ESC pluripotency

PDGF signaling

Chemokine signaling
HMGB1 signaling

IL-8 signaling

Osteoarthritis pathway

(1)

h

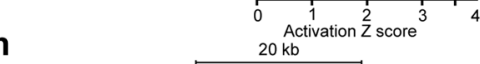

genomic locus START

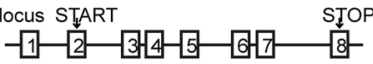
targeted allele homologous recombination

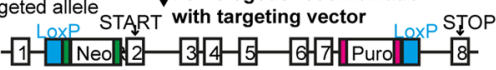
$F R T F R T$
conditional KO allele $\mathrm{Flp}$ recombination$$
\text { START }
$$

-1 1 -

constitutive $\mathrm{KO}$ allele $\mathrm{Cre}$ recombination

$$
0
$$

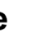

Leukocyte migration Apoptosis of neutrophils Apoptosis of granulocytes

Inflammatory response Cell survival Quantity of cells

$$
\text { Chemotaxis }
$$

of phagocytes
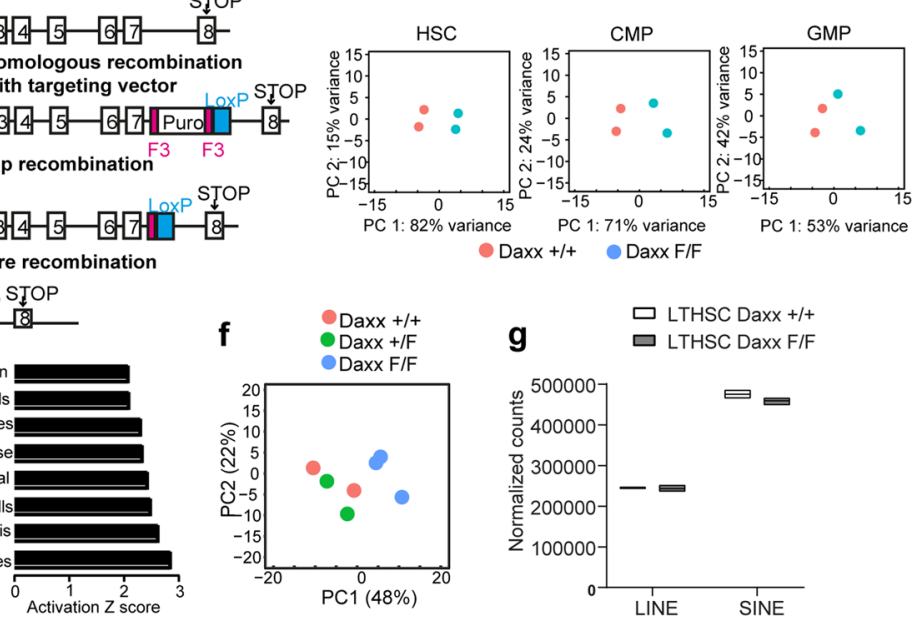
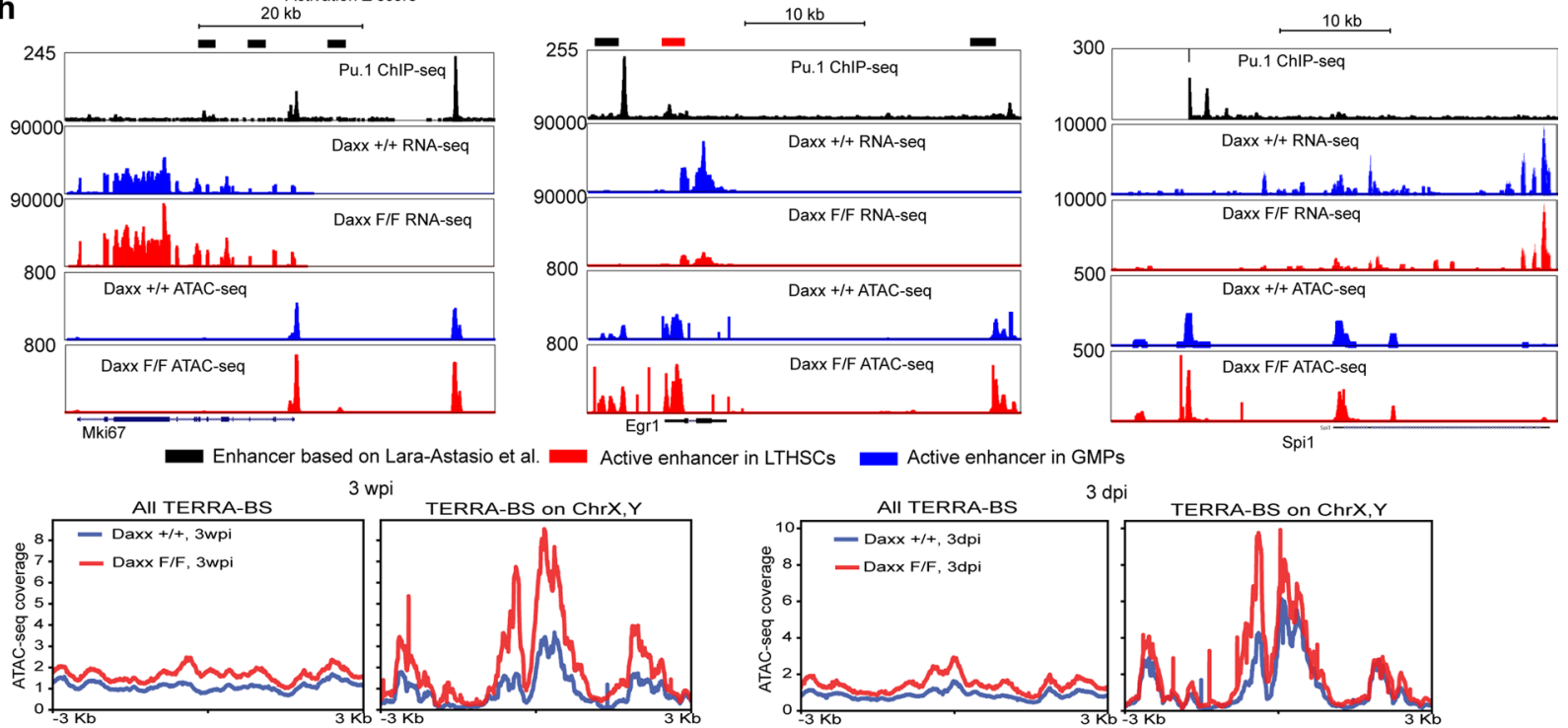

j
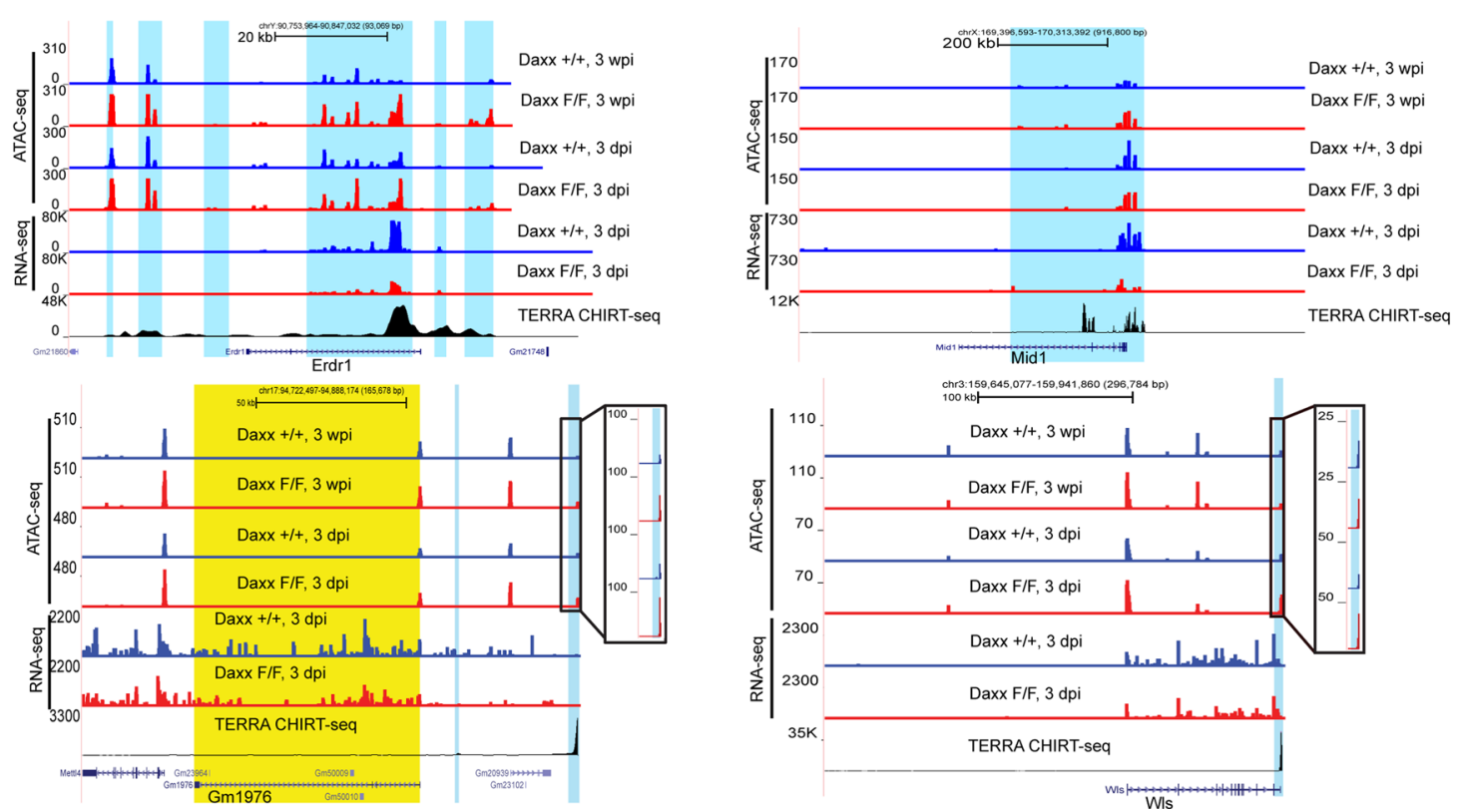

Extended Data Fig. 1 | See next page for caption. 
Extended Data Fig. 1 | Expression of H3.3 chaperones in haematopoietic cells, creating Daxx F/F mice and analysis of LT-HSC ATAC-seq and RNA-seq data. a, Heatmap of Daxx, Atrx and Hira expression levels (data from ${ }^{18}$ ). b. Daxx genomic locus, Daxx floxed locus following homologous recombination, conditional KO allele following deletion of the FRT-flanked or F3-flanked Neomycin (NeoR) and Puromycin (PuroR) resistance gene; Cre-mediated deletion of Daxx exons 2-7. c, PCA for ATAC-seq data (3 w.p.i.; top-500 most variable peaks): displayed principal components (PC) 1 versus 2 . d, Enriched IPA immune system-related canonical pathways for ERV-overlapping enhancer peaks that open upon Daxx KO in LT-HSCs (3 w.p.i.). e, Enriched IPA immune system-related biofunctions for ERV-overlapping enhancer peaks that open upon Daxx KO in LT-HSCs (3 w.p.i.). f, PCA plot for LT-HSC RNA-seq data (3 d.p.i.). g, Counts per million of LINE and SINE elements in LT-HSCs (3 d.p.i.). h, Genome browser tracks of the regulatory region of Spi1, Mki67 and Egr1 (3 d.p.i.). i, ATAC-seq coverage around TERRA-BS across all chromosomes or across sex chromosomes at 3 w.p.i. and 3 d.p.i. $\mathbf{j}$, Genome browser tracks for wild-type (blue) and Daxx KO (red) LT-HSCs (ATAC-seq: 3 w.p.i. and 3 d.p.i.; RNA-seq: 3 d.p.i.) around Erdr1, Mid1, Gm1976 and WIs; TERRA CHIRT-seq based on published data ${ }^{23}$. Light-blue regions are called TERRA binding sites and yellow region highlights the Gm1976 gene. For Gm1976 and WIs boxes show ATAC-seq coverage at the major TERRA binding site. LT.HSC, long-term haematopoietic stem cells; ST.HSC, short-term haematopoietic stem cells; MPP, multipotent progenitor; CMP, common myeloid progenitor; GMP, granulocyte-monocyte progenitor; CLP, common lymphoid progenitor; MEP, megakaryocyte erythroid progenitor. $n=2$ independent biological samples analysed 3 weeks post induction with tamoxifen (c-e, $\mathbf{i}, \mathbf{j}$ ). Analysis of gene expression and chromatin accessibility of LT-HSCs from $n=2$ Daxx $+/+$ and Daxx $+/ F$ as well as $n=3$ Daxx F/F samples collected 3 days post final induction with tamoxifen (f-j). Data are Activation Z-score from IPA Fisher's exact tests with multiple testing adjusted $p$-values $<0.05$ (d, e). Activation $Z$-score $>2$ suggests increased activation of shown biofunctions. Numerical source data provided in Source data. 


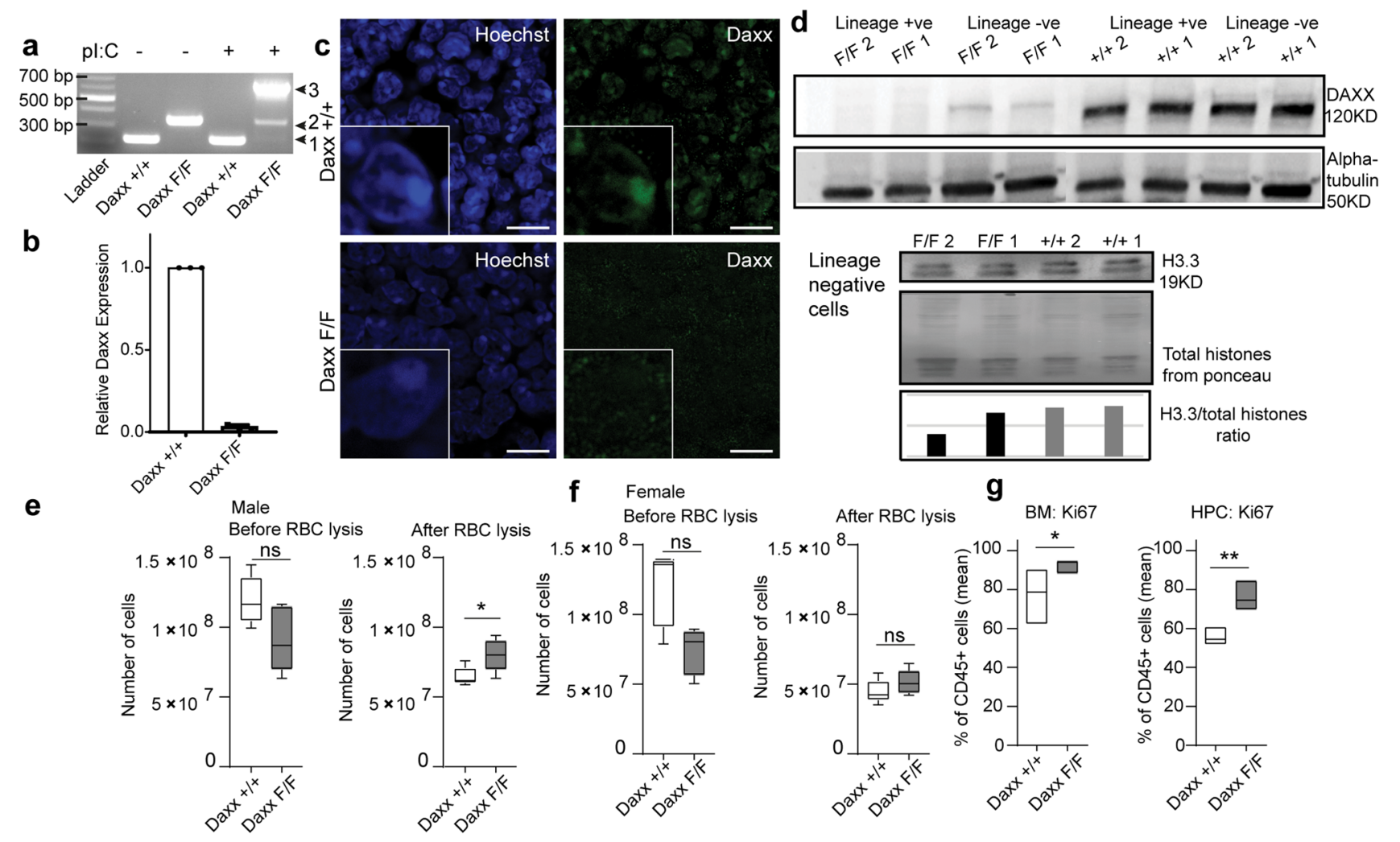

h
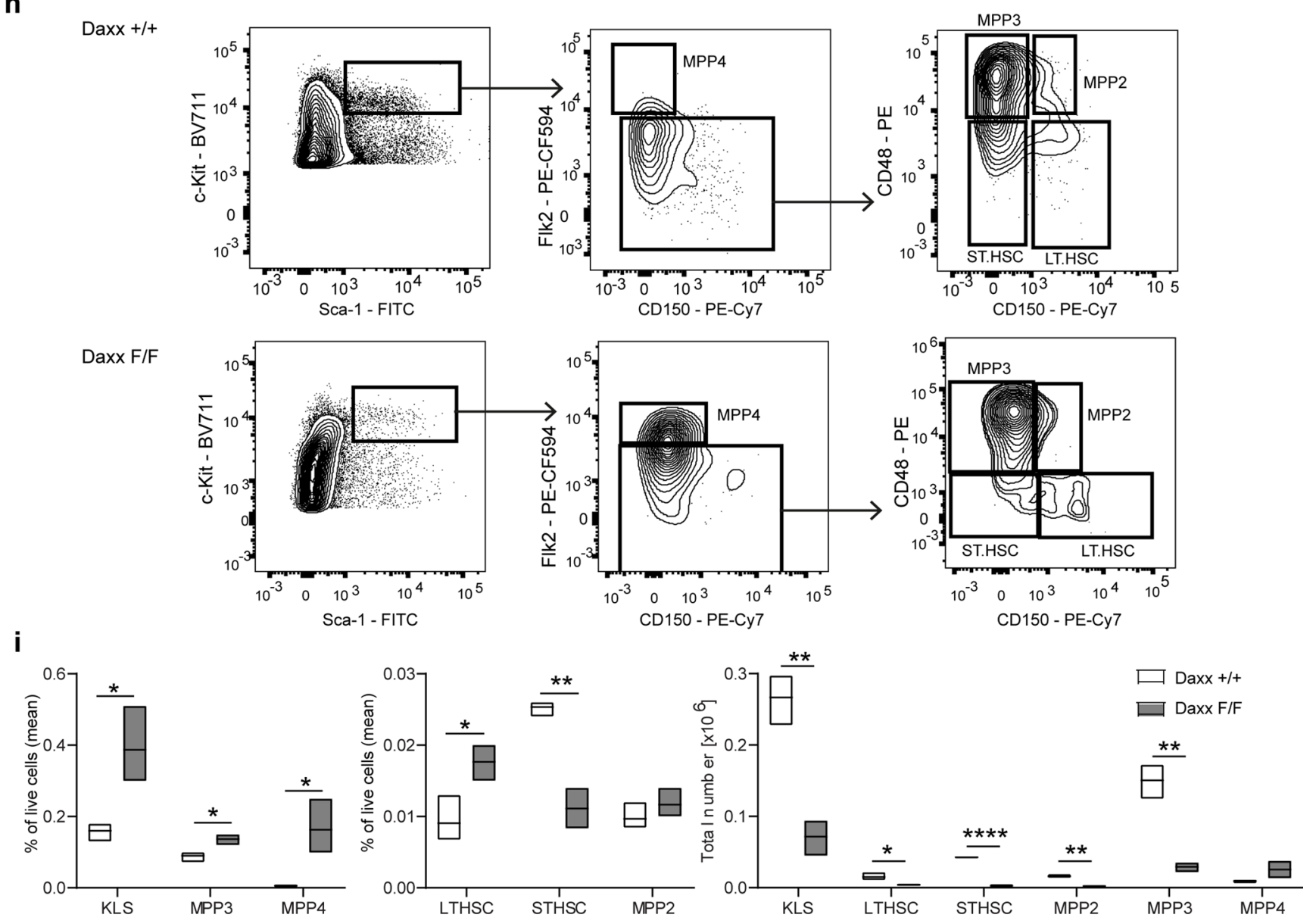

Extended Data Fig. 2 | See next page for caption. 
Extended Data Fig. 2 | Daxx-deficient BM displays increased proliferation and altered stem/progenitor cell frequencies and numbers. a, Genotyping of

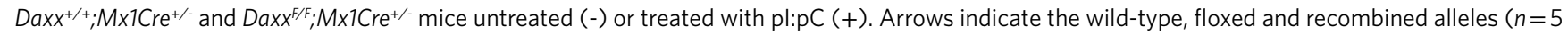
independent experiments). b, Daxx mRNA levels upon pl:pC treatment in bone marrow. Shown is relative expression of Daxx mRNA over Tbp mRNA levels ( $n=3$ mice per genotype). c, IF of Daxx in bone marrow, scale bar $=10 \mu \mathrm{m}(n=4$ mice per genotype, two independent experiments). Inlay image zoomed on representative nuclei. d, Western blot of Daxx, alpha-tubulin and H3.3 in Lineage-negative and Lineage-positive bone marrow cells ( $n=2$ mice per genotype). e, Bone marrow cellularity counts before and after RBC lysis in male mice ( $n=5$ mice per gender, non-parametric Mann-Whitney test, boxand-whiskers plot: $\min$ to $\max$ (whiskers), $25^{\text {th }}$ and $75^{\text {th }}$ percentile and median). f, Bone marrow cellularity counts before and after RBC lysis in female mice ( $n=5$ mice per gender, non-parametric Mann-Whitney test, box-and-whiskers plot: min to max (whiskers), $25^{\text {th }}$ and $75^{\text {th }}$ percentile and median). $\mathbf{g}$, Flow cytometry analysis of Ki-67+ cells in BM and HPCs ( $n=5$ mice per genotype, repeated in two independent experiments, non-parametric Mann-Whitney test). $\mathbf{h}, \mathbf{i}$, Flow cytometry analysis of HSC and MPP populations ( $n=3$ mice per genotype, repeated in two independent experiments, Student's t-test). BM, bone marrow; HPC, haematopoietic progenitor cells; HSC, haematopoietic stem cell; MPP, multipotent progenitors. Data in box plots are mean and min to max. ns, not significant; ${ }^{\star} P<0.05$, ${ }^{\star \star} P<0.01$, ${ }^{\star \star \star} P<0.001,{ }^{\star \star \star \star} P<0.0001$. Exact $p$-values and numerical source data can be found in the accompanying source data. Unprocessed blots provided in Source data. 
a
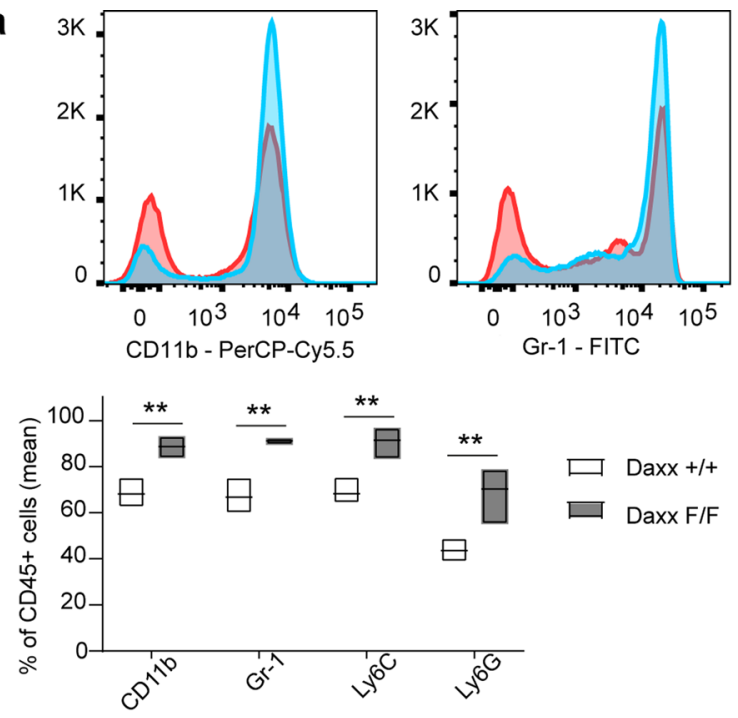
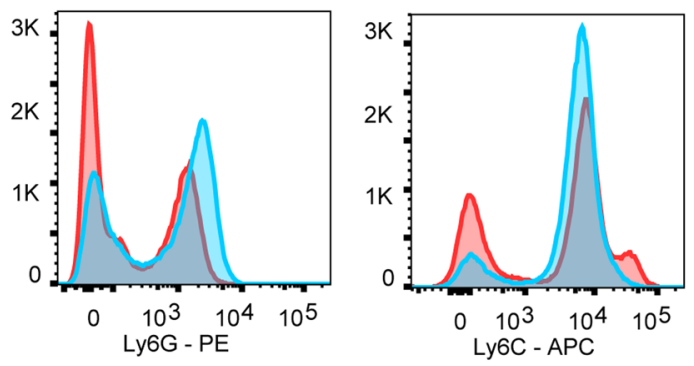

b

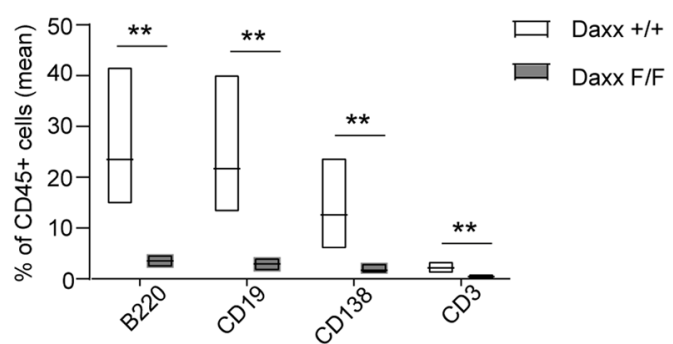

C

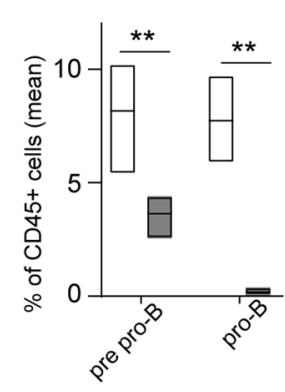

$\operatorname{Daxx}+/+$

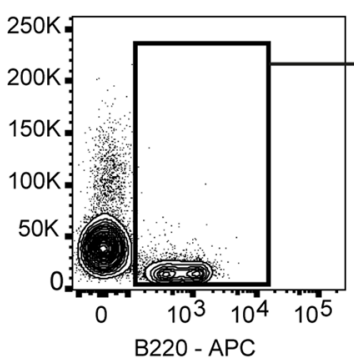

Daxx F/F

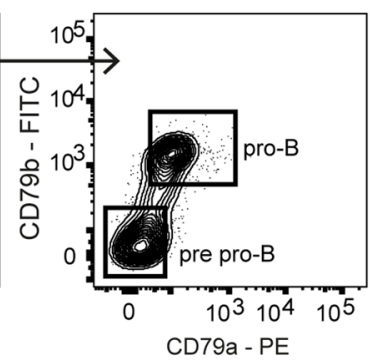

Daxx F/F

$\operatorname{Daxx}+/+$ d

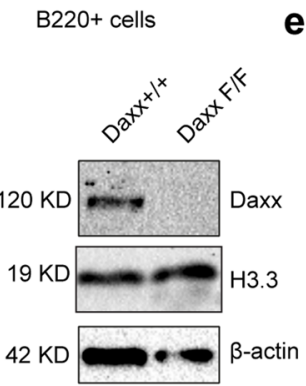

e

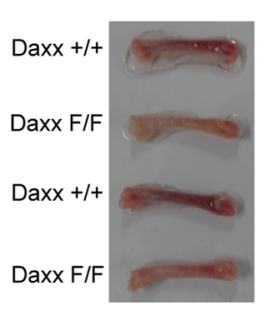

f

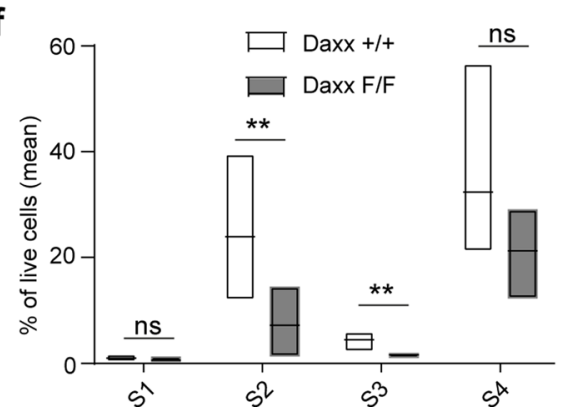

Extended Data Fig. 3 | Daxx deficiency results in myeloid/lymphoid imbalance in the bone marrow. a, Flow cytometry analysis of myeloid cell surface markers ( $n=6$ mice per genotype, repeated in three independent experiments). b. Flow cytometry analysis of lymphoid cell surface markers ( $n=6$ mice per genotype, repeated in three independent experiments). c, Frequencies of B cell progenitors $(n=6$ mice per genotype, repeated in three independent experiments. d, Western blot of Daxx, H3.3 and corresponding $\beta$-actin control in isolated B220 $0^{+}$cells from bone marrow $(n=3$ mice per genotype, repeated in two independent experiments). e, Bones isolated from two Daxx ${ }^{+/+} ; M \times 1 C r e$ and $D a x x^{F / F} ; M x 1 C r e ~ m i c e . ~ f$, Frequencies of erythroblast populations ( $n=6$ mice per genotype, repeated in three independent experiments. Data in box plots are mean and min to max. ns, not significant; ${ }^{\star} P<0.05, d{ }^{\star *}$ $P<0.01,{ }^{\star \star \star} P<0.001,{ }^{\star \star \star \star} P<0.0001$, Wilcoxon rank test. Exact $p$-values and numerical source data can be found in the accompanying source data. Source image file provided in Source data. 


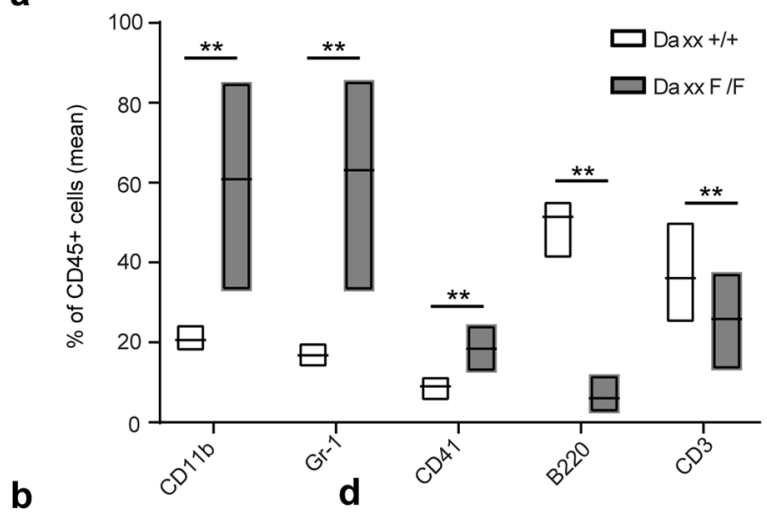

b
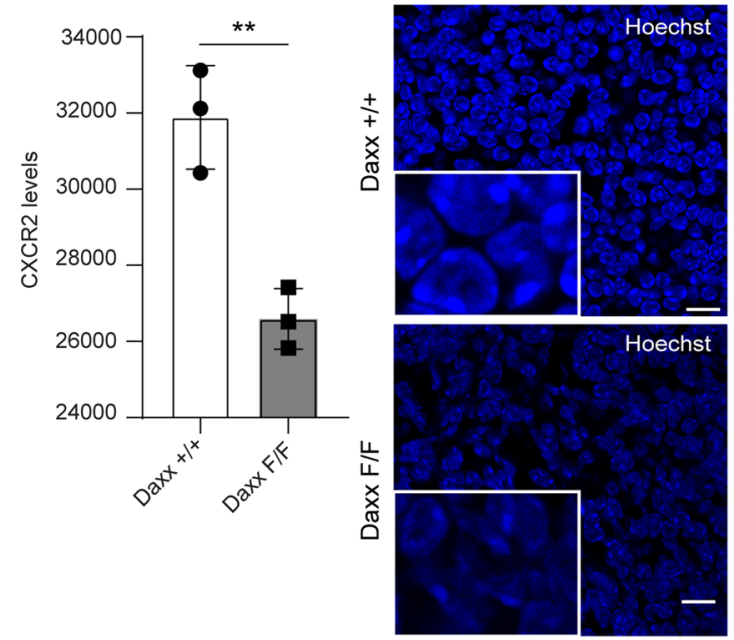

e

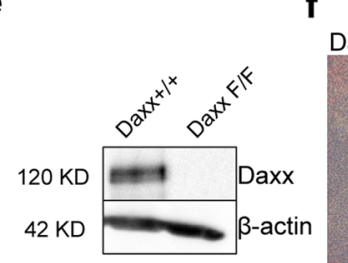

h

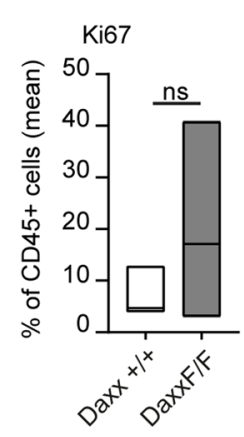

i

\section{C}
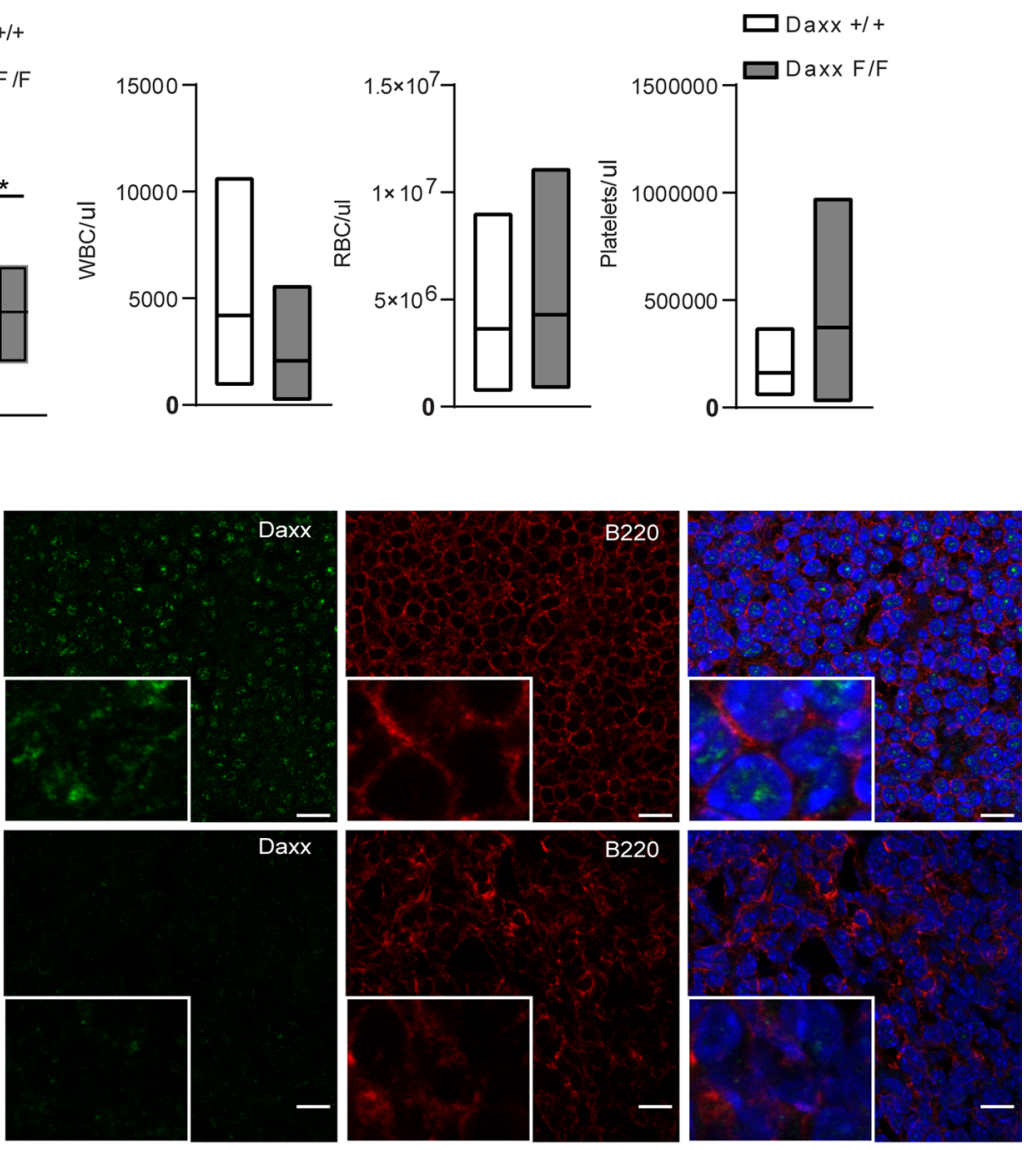

g BM Daxx F/F (Cre -/-)
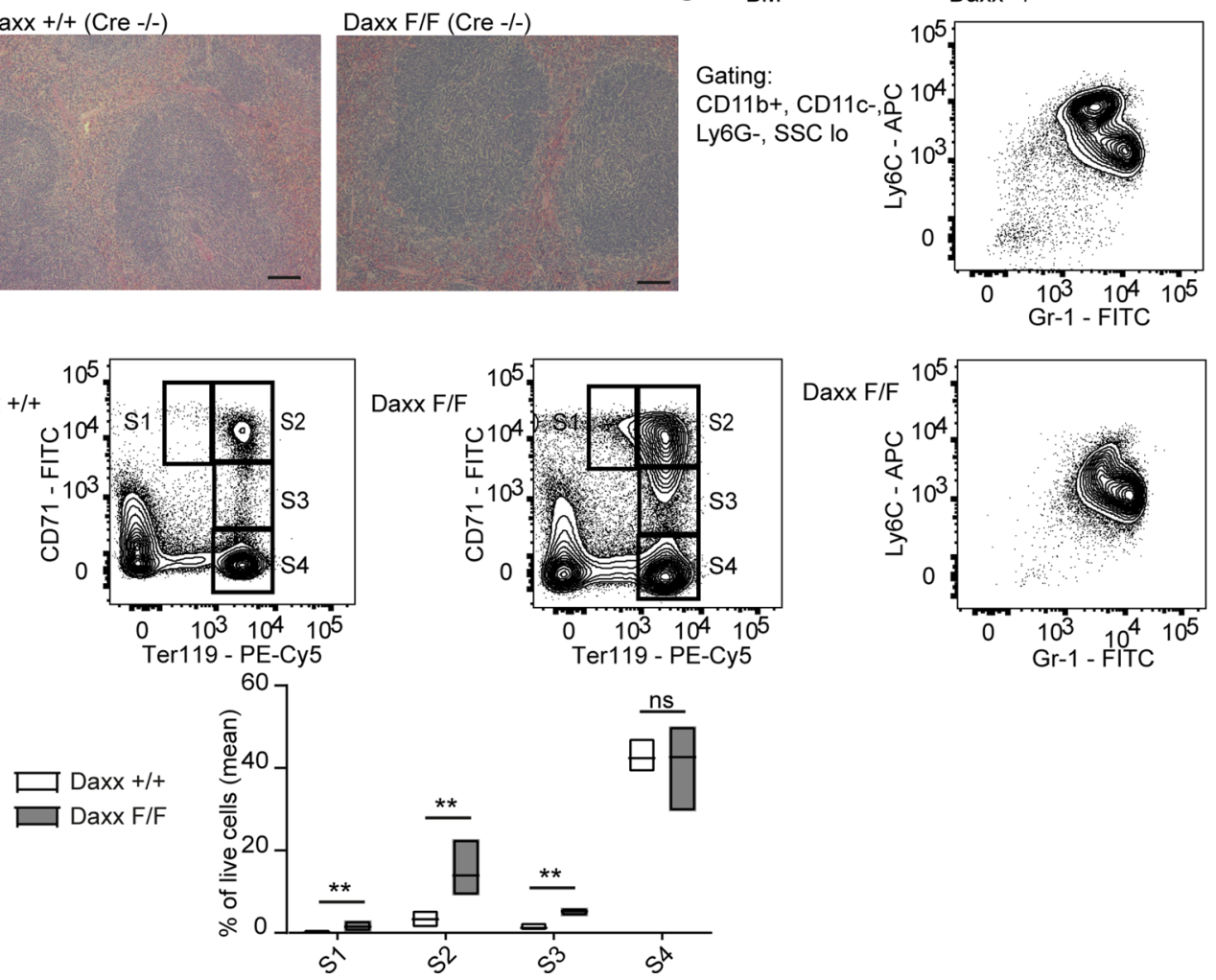

ns 
Extended Data Fig. 4 | Loss of Daxx leads to neutrophilia and B-cell lymphopenia in the periphery. a, Flow cytometry analysis of peripheral blood collected 4 weeks post induction ( $n=6$ mice per genotype, repeated in three independent experiments). $\mathbf{b}, C x c r 2$ levels in mature neutrophils of the blood ( $n=3$ mice per genotype, Student's t-test). c, Quantification of WBC, RBC and platelets using Sysmex ( $n=3$ mice per genotype). d, IF images of spleen stained for Daxx and B220 ( $n=2$ mice per genotype, repeated in two independent experiments). Scale bar indicates $10 \mu \mathrm{m}$. e, Western Blot of Daxx protein in spleen ( $n=3$ mice per genotype, repeated in three independent experiments). $\mathbf{f}, \mathrm{H} \& \mathrm{E}$ stain of spleen sections in Cre-negative animals, scale bar $=100 \mu \mathrm{m}(n=2$ mice per genotype). $\mathbf{g}$, Representative flow cytometry plots showing Gr-1+/Ly6C+ populations in bone marrow upon CD11b+, CD11c', Ly6G ${ }^{-}, \mathrm{SSC}^{10}$ gating. $\mathbf{h}$, Frequencies of $\mathrm{Ki}^{6} 7^{+}$cells in spleen ( $n=5$ mice per genotype, repeated in two independent experiments). $\mathbf{i}$, Frequencies of erythroblast populations in spleen ( $n=6$ mice per genotype, repeated in three independent experiments). Data in box plots are mean and min to max. ns, not significant; ${ }^{\star} P<0.05,{ }^{\star \star} P<0.01,{ }^{\star \star \star} P<0.001,{ }^{\star \star \star \star} P<0.0001$, Wilcoxon rank test if not otherwise noted. Exact $p$-values and numerical source data can be found in the accompanying source data. Source image file provided in Source data. 
a
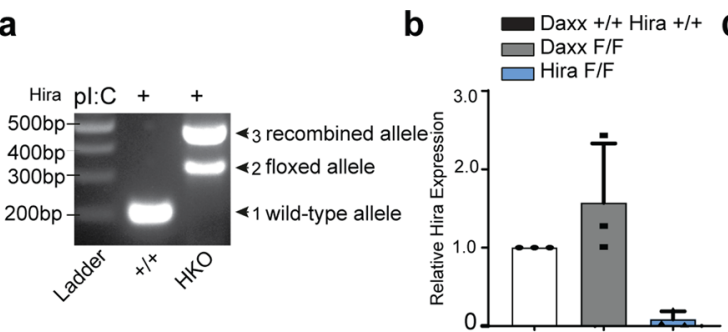

C

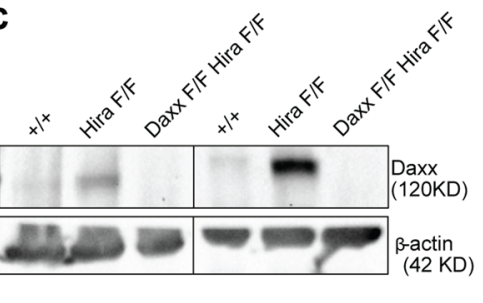

d

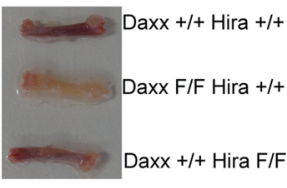

$\square$ Daxx +/+ Hira +/+ $\square$ Daxx F/F

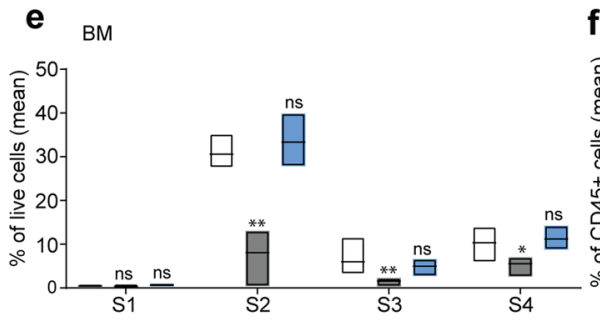

f $\mathrm{BM}$

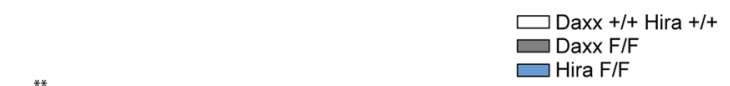

h BM: Neutrophils i Spleen: Neutrophils j

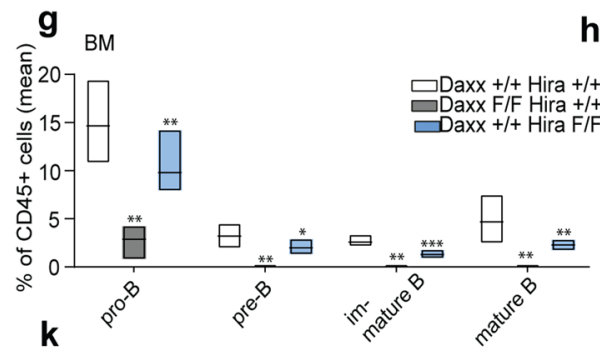

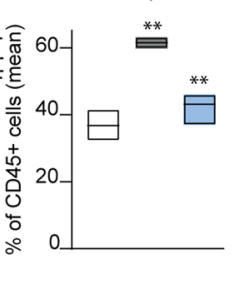
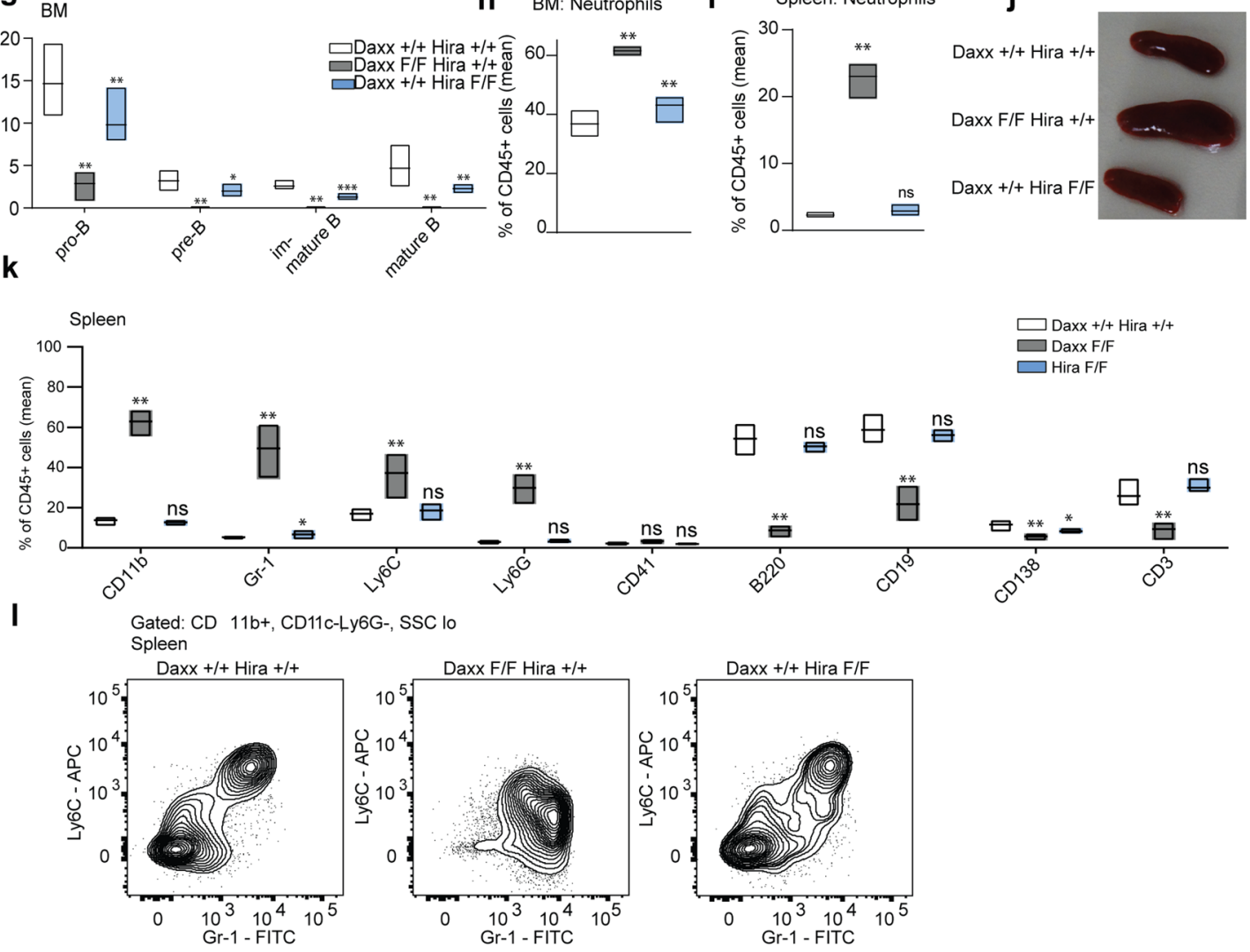

m
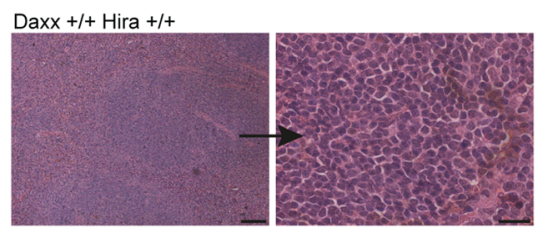

Daxx +/+ Hira F/F

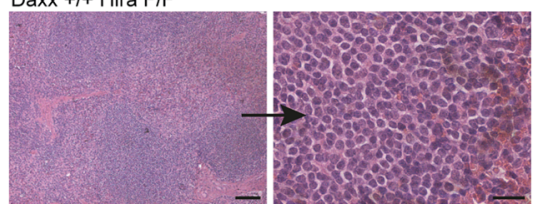

Daxx F/F Hira +/+

曰 $\stackrel{\text { ns }}{日} \quad$ ns
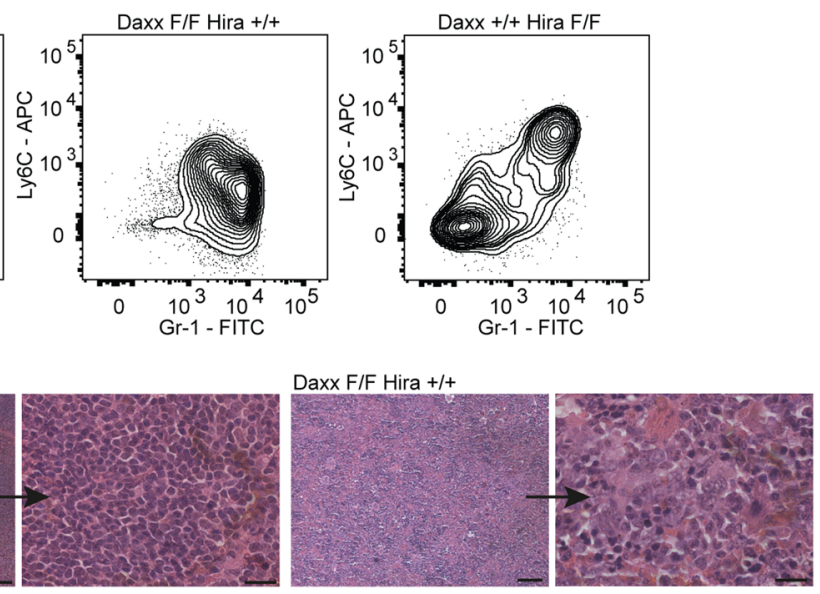
Extended Data Fig. 5 | Loss of the H3.3 chaperone Hira has marginal effects on haematopoiesis. a, Representative images of genotyping results using primers to detect WT, floxed and recombined bands of Hira in bone marrow cells ( $n=3$ independent experiments). $\mathbf{b}$, Hira mRNA levels upon pl:pC treatment in bone marrow cells. Shown is relative expression of Hira mRNA over Tbp mRNA levels ( $n=3$ mice per genotype). c, Representative Western blots for Daxx and $\beta$-Actin in total bone marrow of Daxx ${ }^{+/+}$, Hira ${ }^{\mathrm{F} / \mathrm{F}}$ and Daxx/Hira double KO Mx1Cre mice $(n=3$ mice per genotype, repeated in two independent experiments). d, Bones of WT, Daxx KO and Hira KO mice. e, Frequencies of erythroblast populations in bone marrow (WT mice $n=9$; Daxx KO $n=3$; Hira KO $n=6$ ). f, Flow cytometry analysis of mature cell markers in bone marrow (WT mice $n=9$; Daxx KO $n=3$; Hira KO $n=6$ ). $\mathbf{g}$, Flow cytometry analysis of $B$ cells in bone marrow (WT mice $n=9$; Daxx KO $n=3$; Hira KO $n=6$ ). $\mathbf{h}$, Frequencies of neutrophils in bone marrow (WT mice $n=9$; DKO $n=3 ;$ HKO $n=6$ ). $\mathbf{i}$, Frequencies of neutrophils in spleen (WT mice $n=9 ;$ Daxx KO $n=3 ;$ Hira KO $n=6$ ). $\mathbf{j}$, Spleens isolated from WT, Daxx KO and Hira KO mice. k, Flow cytometry analysis of mature cells in spleen (WT mice $n=9$; Daxx KO $n=3$; Hira KO $n=6$ ). I, Flow cytometry plots gated on monocyte- and macrophage-like populations in spleen. $\mathbf{m}, \mathrm{H} \& \mathrm{E}$ stain of spleen sections ( $n=3$ mice per genotype), scale bar $=100 \mu \mathrm{m}$. Higher magnification, scale bar $=20 \mu \mathrm{m}$. Data produced 3 weeks post pl:pC treatment. Data in box plots are mean and min to max. ns, not significant; ${ }^{\star} P<0.05$, ${ }^{\star \star} P<0.01,{ }^{\star \star \star} P<0.001,{ }^{\star \star \star \star} P<0.0001$, Wilcoxon rank test. Exact $p$-values and numerical source data can be found in the accompanying source data. Source image file provided in Source data. 

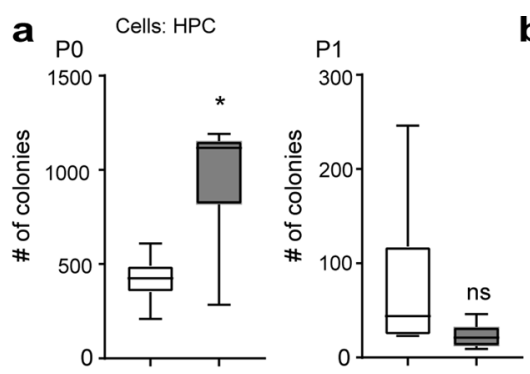

b Cells: LT-HSCs

C

Cells: LT-HSCs

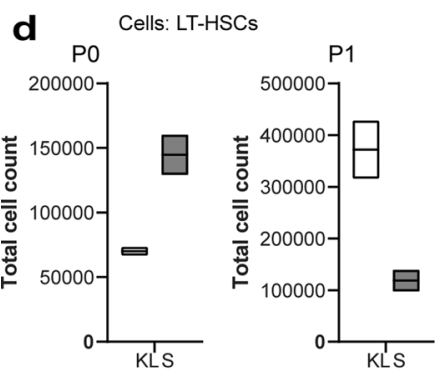

$\underbrace{200}_{\mathrm{P} 0}$

口 $\operatorname{Daxx}+/+$

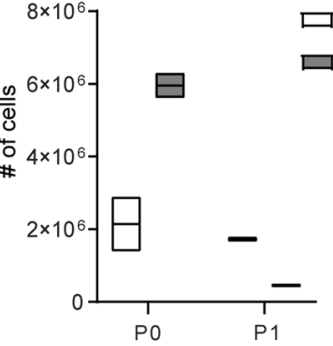

e

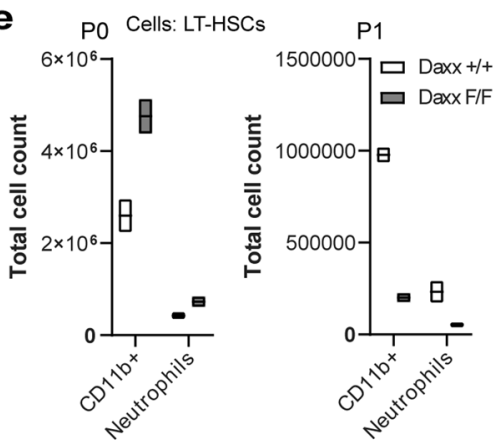

f

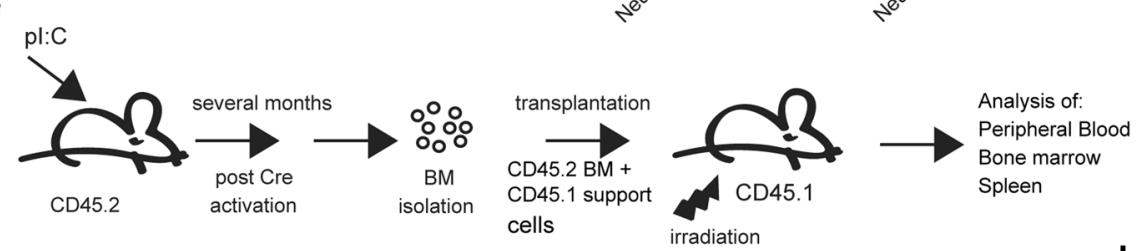

g

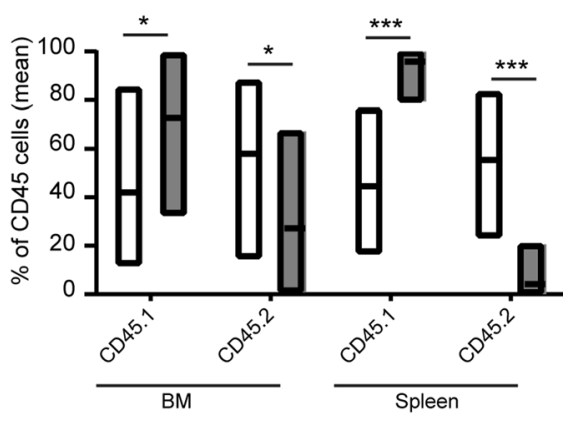

rim

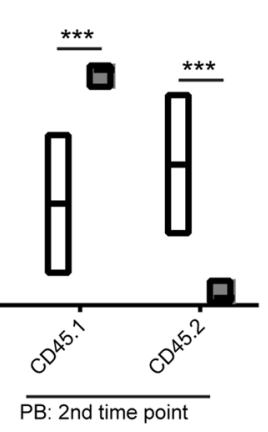

h BM

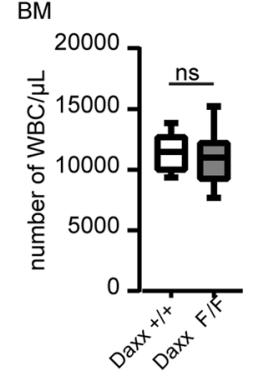

i PB: 1st time point
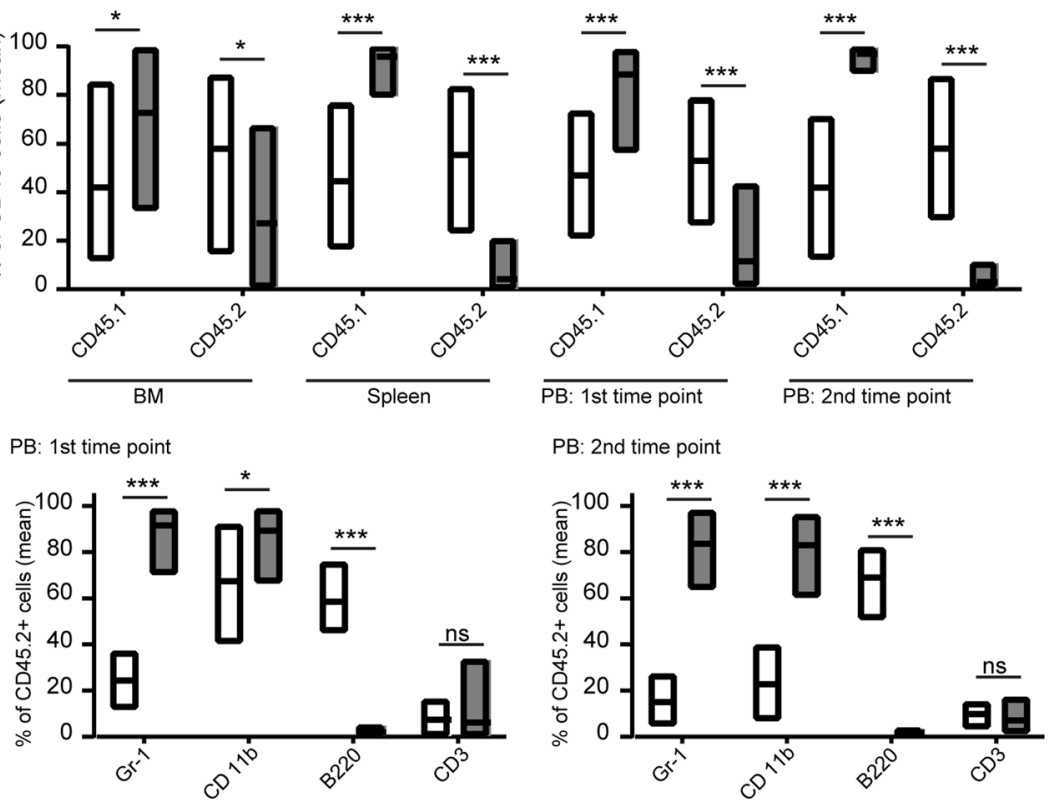

j $\mathrm{BM}$

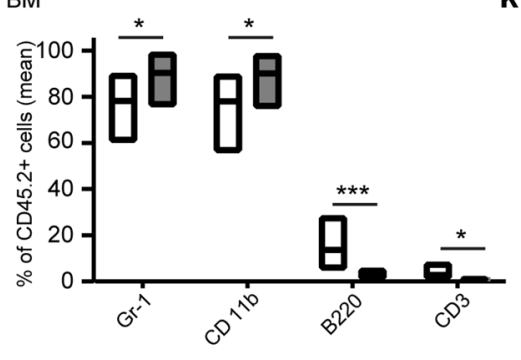

k $\mathrm{BM}$

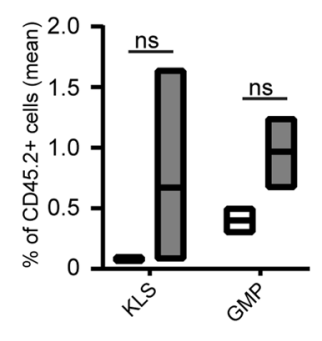

I Spleen

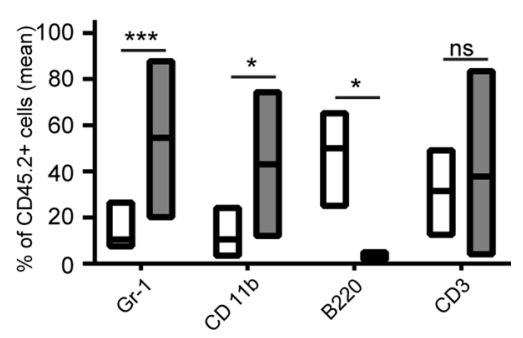

Extended Data Fig. 6 | See next page for caption. 
Extended Data Fig. 6 | The haematopoietic phenotype of Daxx deletion is due to a cell-intrinsic defect. a, Number of colonies after first plating (PO) and first passage (P1) using haematopoietic progenitor cells ( $n=5$ mice per genotype, repeated in three independent experiments, box-and-whiskers plot: $\min$ to $\max , 25^{\text {th }}$ to $75^{\text {th }}$ percentile and median). b. Number of colonies after first plating (PO) and first passage (P1) using LT-HSCs ( $n=2$ mice per genotype). c, Number of cells after first plating (PO) and first passage (P1) using LT-HSCs ( $n=2$ mice per genotype). d, Number of KLS cells and e, number of CD11b + cells and neutrophil-like cells ( Ly6C + ,Ly6G + ) after first plating (PO) of LT-HSCs and first passage (P1; $n=2$ mice per genotype). f, Schematic representation of the experimental set-up to generate bone marrow chimeras. $\mathbf{g}$, Frequencies of CD45.1 $1^{+}$and CD45.2+ cells in transplanted mice $(n=8$ mice per genotype). $\mathbf{h}, \mathrm{WBC}$ counts in total bone marrow ( $n=8$ mice per genotype, box-and-whiskers plot: $\min$ to $\max , 25^{\text {th }}$ to $75^{\text {th }}$ percentile and median). i, Flow cytometry analysis in peripheral blood of transplanted mice at two different time points: $1^{\text {st: }}$ 4-5 weeks post transplantation; $2^{\text {nd }}$ pooled data from two independent experiments collected at 8 or 12 weeks post transplantation ( $n=8$ mice per genotype). j, Frequencies of mature CD45.2+ blood cells in bone marrow of recipient mice ( $n=8$ mice per genotype, repeated in three independent experiments). $\mathbf{k}$, Frequencies of CD45.2+ KLS and GMPs in bone marrow ( $n=4$ mice per genotype, repeated in two independent experiments). I, Flow cytometry analysis of CD45.2+ mature blood cells in spleen of recipient mice ( $n=8$ mice per genotype, repeated in three independent experiments). BM, bone marrow; PB, peripheral blood; KLS, $c-K i^{p o s}$, lineage ${ }^{\text {neg }}$, Sca-1 $\uparrow^{\text {pos; }}$ GMP, granulocyte-monocyte progenitor; PO, first plating; P1, first passage. Data in box plots are mean and min to max. ns, not significant; ${ }^{\star} P<0.05,{ }^{\star \star} P<0.01,{ }^{\star \star \star} P<0.001,{ }^{\star \star \star \star} P<0.0001$, Wilcoxon rank test. Exact $p$-values and numerical source data can be found in the accompanying source data. 
a

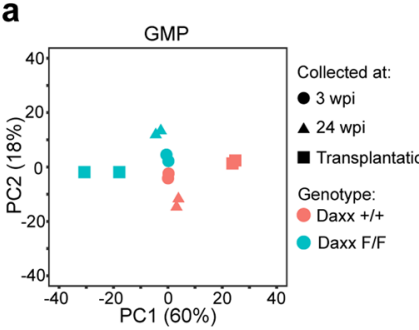

C

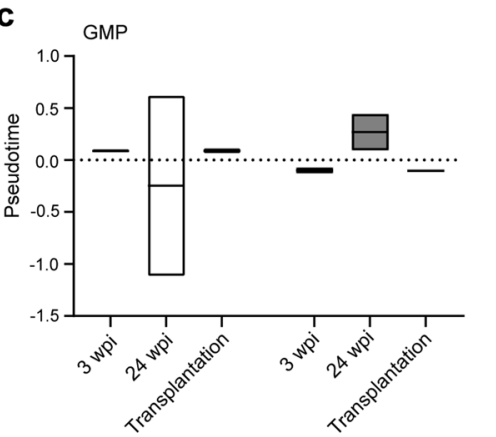

e

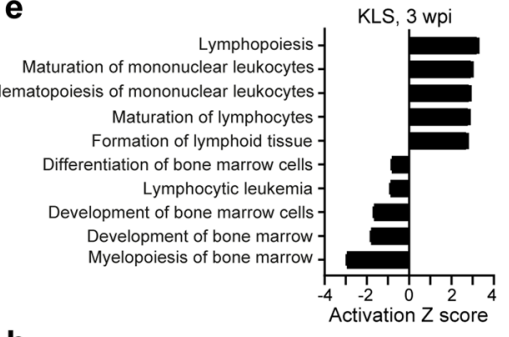

h
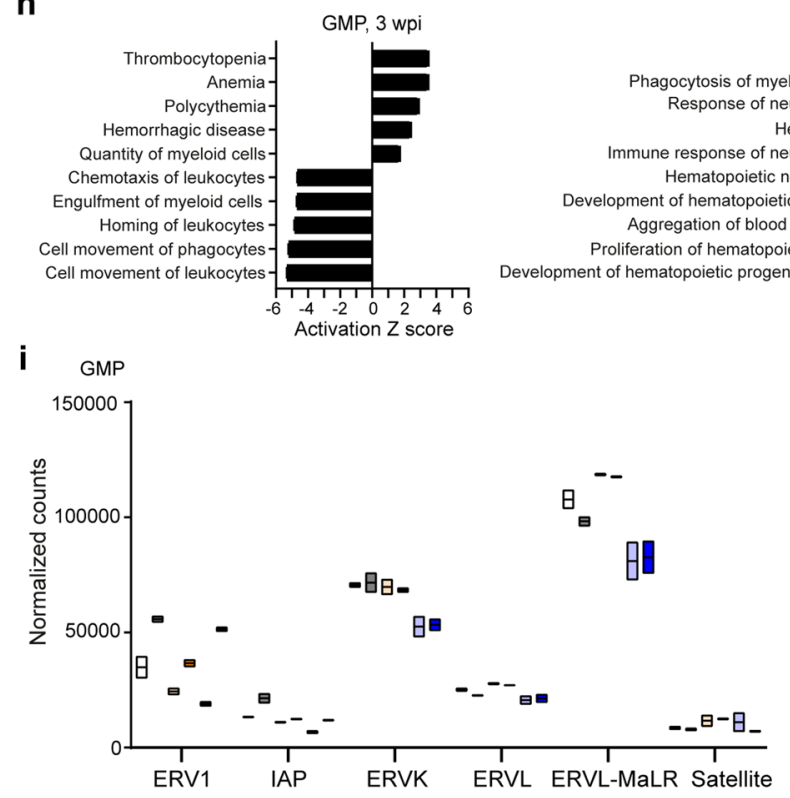

$\square$ Daxx +/+ 3 wpi $\square$ Daxx F/F 3 wpi

$\square$ Daxx +/+ 24 wpi

$\square$ Daxx F/F 24 wpi $\square \begin{aligned} & \text { Daxx +/+ } \\ & \text { Transplantation }\end{aligned}$

$\square \operatorname{Daxx}+/+$

Daxx F/F

f
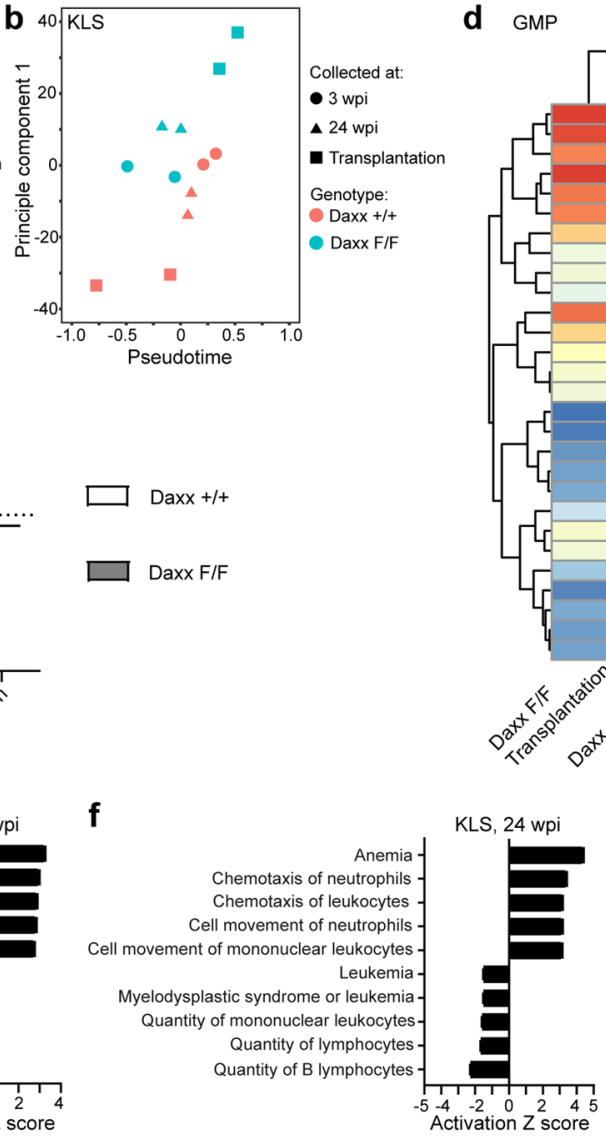

g
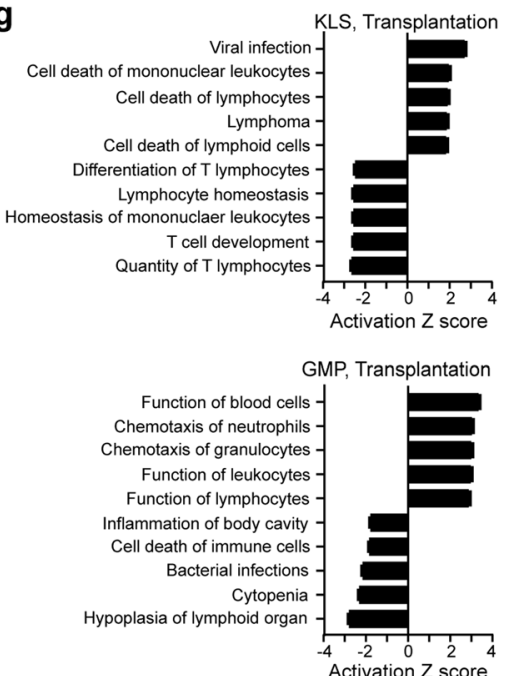

j GMP

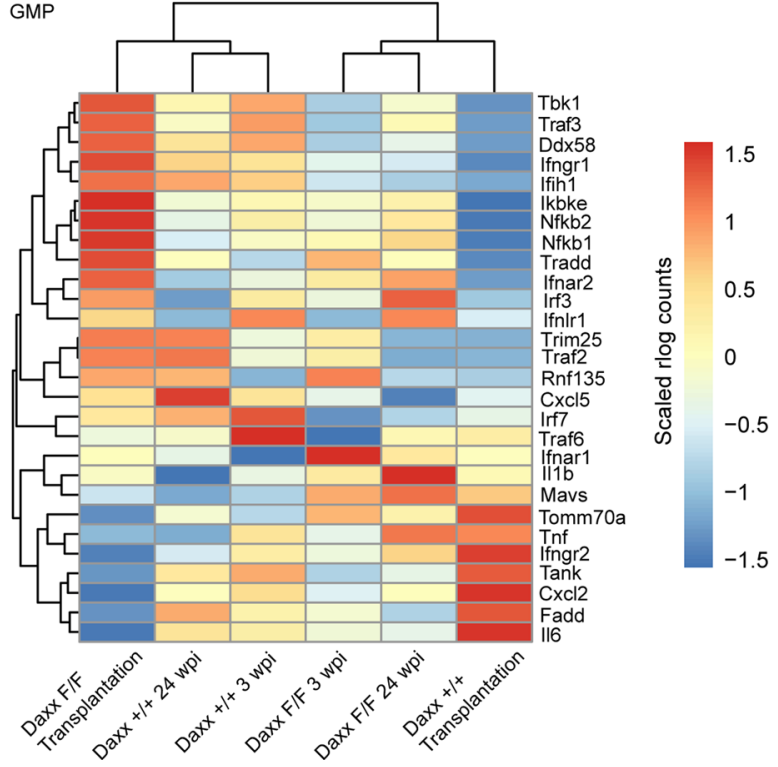

Extended Data Fig. 7 | See next page for caption. 


\section{NATURE CELL BIOLOGY}

Extended Data Fig. 7 | Gene expression changes in Daxx-deficient haematopoietic progenitors. a, PCA of the top-500 most variable genes for GMPs.

b, Trajectory of gene expression of KLS collected at 3 w.p.i., 24 w.p.i. or from transplanted animals. c, Boxplot showing mean pseudotime for each sample group for GMPs ( $n=2$ mice per genotype). d, Heatmap showing scaled expression of selected transcription factors and regulators involved in blood cell differentiation ( $n=2$ mice per genotype). e, Top 5 haematologic biofunctions and diseases altered at 3 w.p.i. in KLS Daxx KO cells. f, Top 5 haematologic biofunctions and diseases altered at 24 w.p.i. in KLS Daxx KO cells. g, Top 5 haematologic biofunctions and diseases altered in KLS Daxx KO cells isolated from transplanted animals. h, Top 5 haematologic biofunctions and diseases altered in GMP Daxx KO cells collected at 3 w.p.i., 24 w.p.i. and from transplanted animals. i, Boxplots of mean normalized read counts of ERV subtypes and satellite repeats ( $n=2$ mice per genotype) in GMP cells. j, Heatmap showing scaled expression of interferon response genes and the dsRNA recognition machinery for GMPs. Data in box plots are min to max and mean. Numerical source data can be found in the accompanying source data. 
a

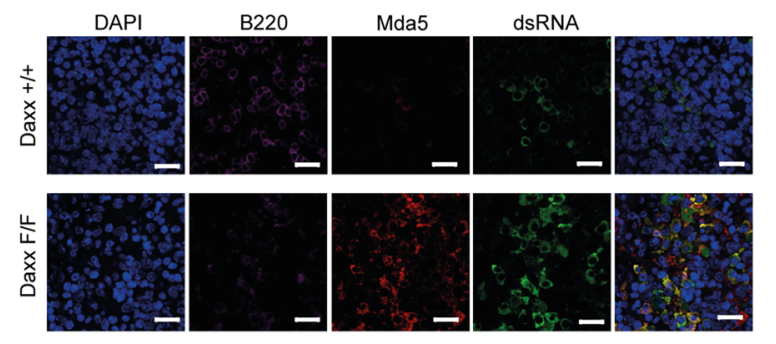

b

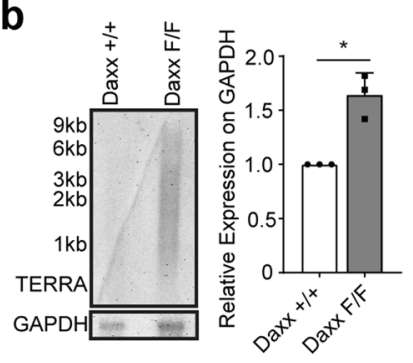

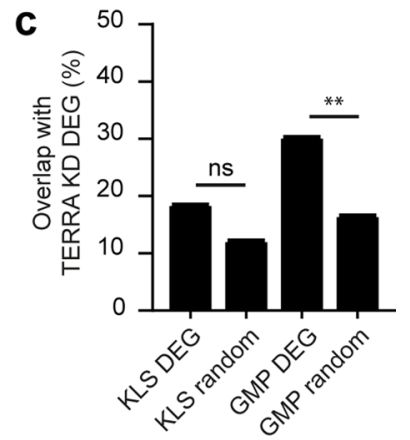

Daxx $+/+$ Pu. 1 F/F
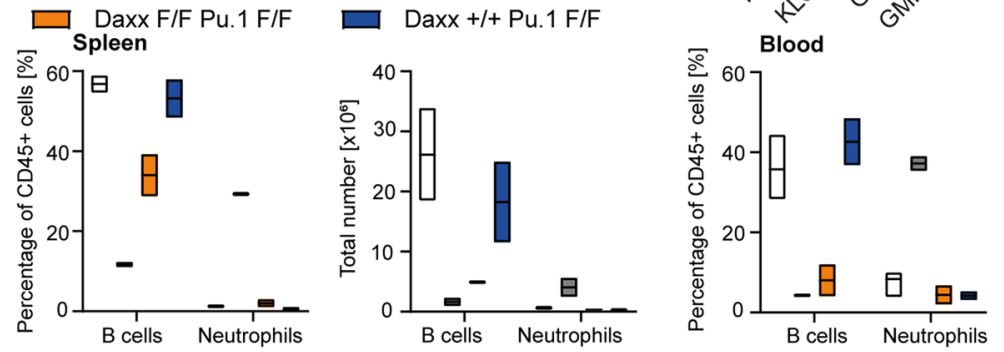

e
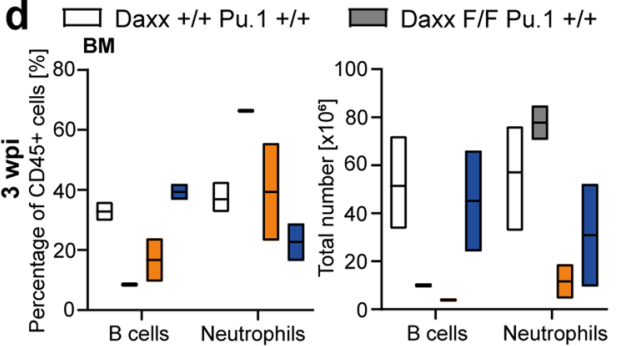

$\square \operatorname{Daxx}+/+$ Pu. $1+/+$
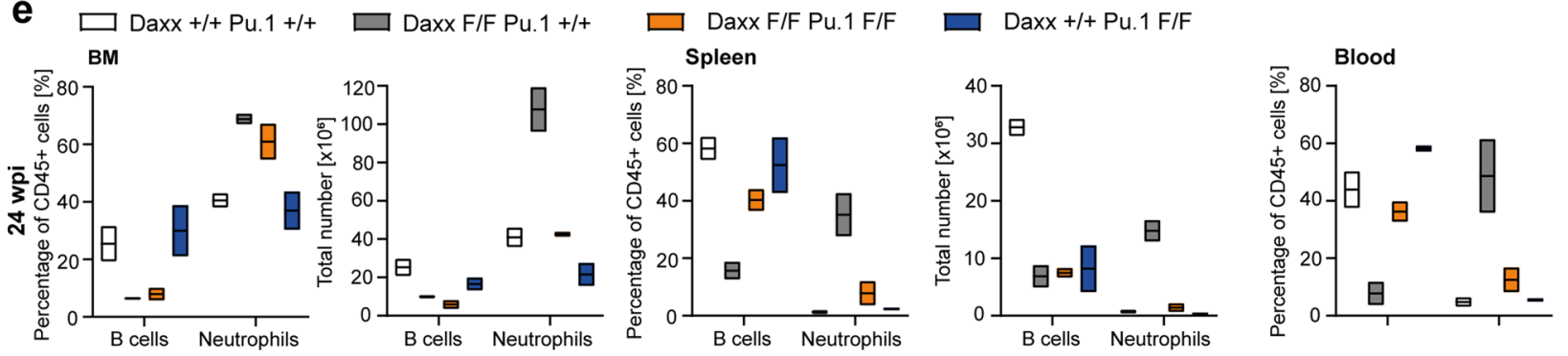

Extended Data Fig. 8 | Changes in the RNA-sensing machinery and TERRA expression following Daxx loss. a, IF images of spleens stained for B220, Mda5 and dsRNA ( $n=2$ independent experiments), scale bar $=20 \mu \mathrm{m}$. b. Northern blot autoradiograph showing TERRA levels and quantification analysis ( $n=3$ mice per genotype, unpaired t-test with Welch's correction). c, Barplot showing the overlap of differentially expressed genes in TERRA knockdown cells with DEG in our KLS and GMP Daxx KO datasets (transplantation) compared to random genes (Fisher's Exact Test; ${ }^{\star \star} p$-value < 0.01, ns, not significant). d, Quantification of B cells and neutrophils in bone marrow, spleen and peripheral blood at 3 weeks post induction ( $n=2$ mice per genotype). e, Quantification of B cells and neutrophils in bone marrow, spleen and peripheral blood at 24 weeks post induction ( $n=2$ mice per genotype). Data in box plots are min to max and mean. Exact $p$-values and numerical source data can be found in the accompanying source data. 
a

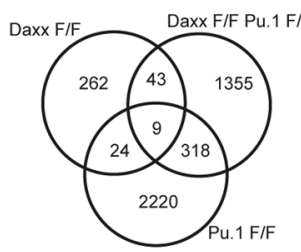

b

GMP

Daxx F/F Pu.1 F/F
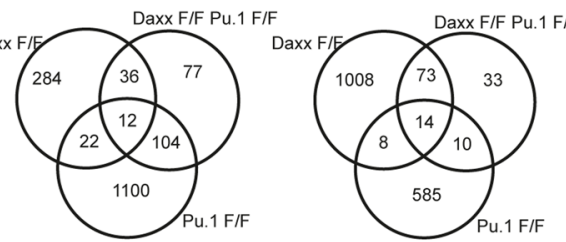

d

GMP

e
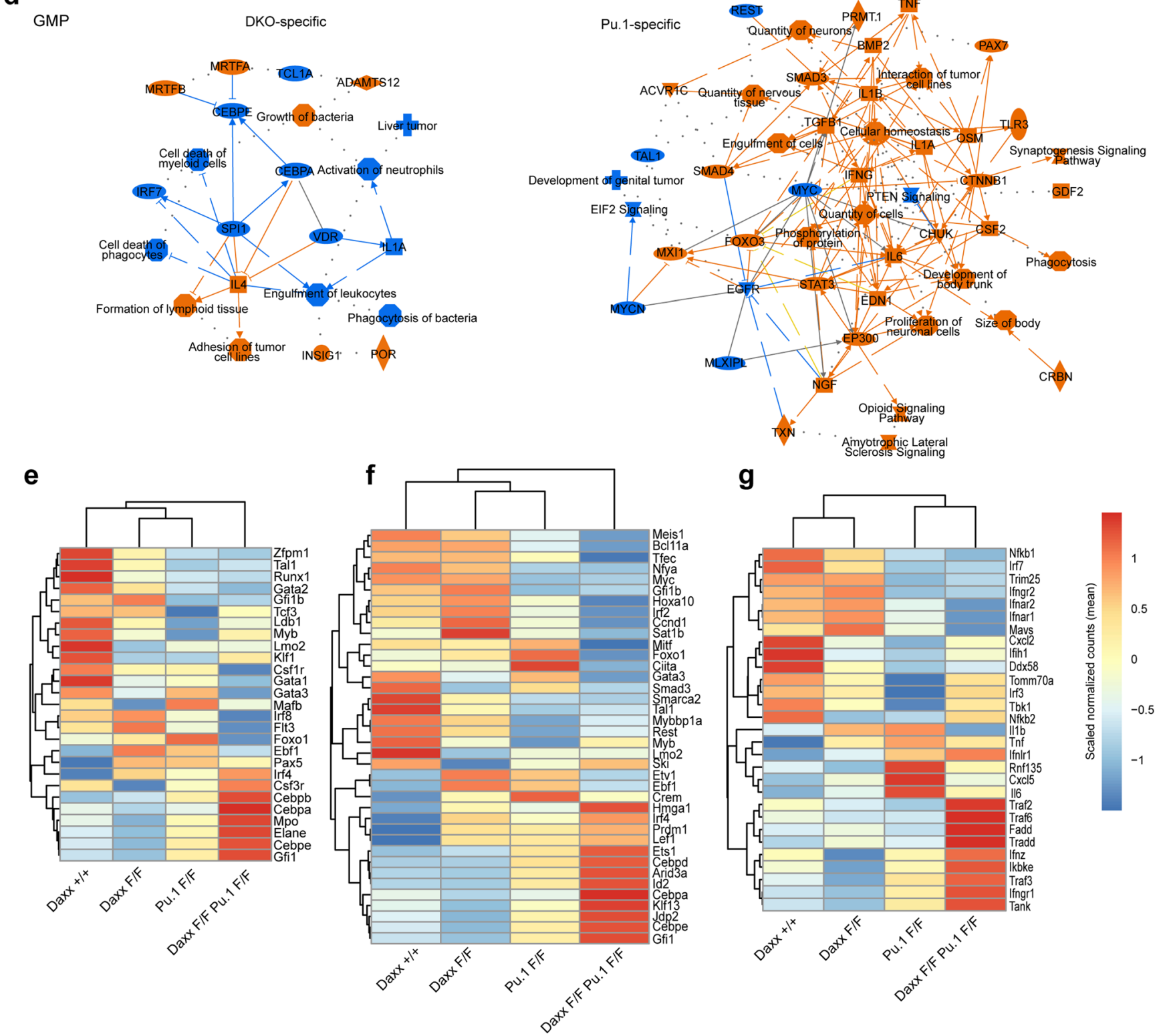

C

$$
\text { KLS DKO-specific }
$$

$\underset{\text { Central nervous system }}{\stackrel{\text { PBX }}{\longrightarrow}}$

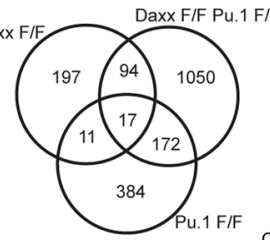

Cell mot

Anaplastic al Senescince of cells MYBL2

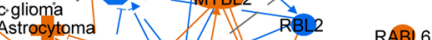

High grade
astroyngama

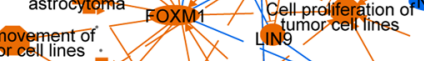

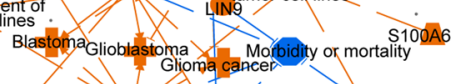

Migraion offumor VEGG
cellines

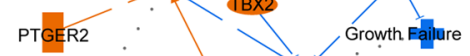

Actin Cytosiseleteton
Signaling
NEE212

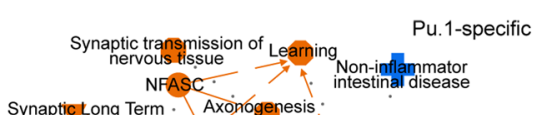

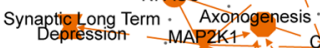

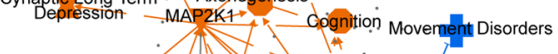

Quantity of neurons - Neufititogenesis / Formation of

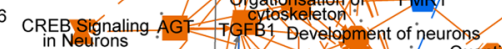

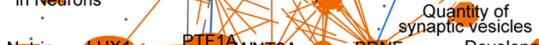

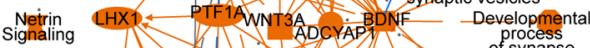

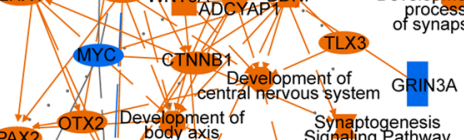

PAX2 OTX2 Develooment of

Development. PAXO RES

BX IIXXP. LAMTOR1 . . . NONO

Extended Data Fig. 9 | See next page for caption. 
Extended Data Fig. 9 | RNA-seq data from wild-type, Daxx single KO, Daxx Pu.1 double KO and Pu.1 single KO KLS and GMP cells collected at 3 w.p.i. a, Venn diagrams of differentially upregulated and downregulated genes in comparison to wild-type KLS. $\mathbf{b}$, Venn diagrams of differentially upregulated and downregulated genes in comparison to wild-type GMP cells. c, IPA graphical summary for KLS Daxx Pu.1 double knock-out specific and Pu.1 single KO specific gene expression changes. d, IPA graphical summary for GMP Daxx Pu.1 double knock-out specific and Pu.1 single KO specific gene expression changes. e, Heatmap showing scaled expression of selected transcription factors and regulators involved in blood cell differentiation ( $n=2$ mice per genotype). f, Heatmap showing scaled expression of transcription factors regulated by Pu.1 ( $n=2$ mice per genotype). g, Heatmap showing scaled expression of interferon response genes and the dsRNA recognition machinery ( $n=2$ mice per genotype). Numerical source data provided in Source data. 
a

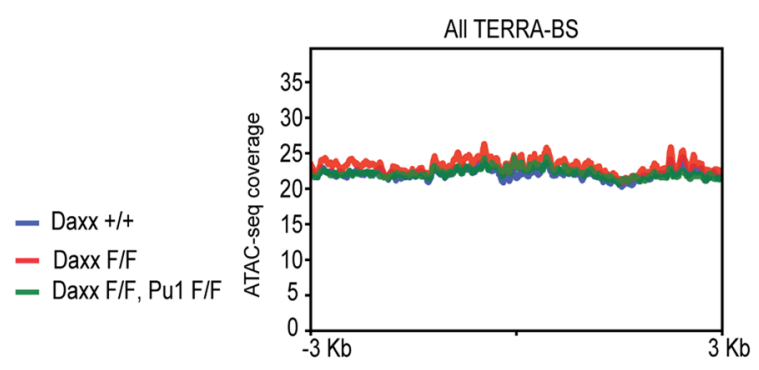

b

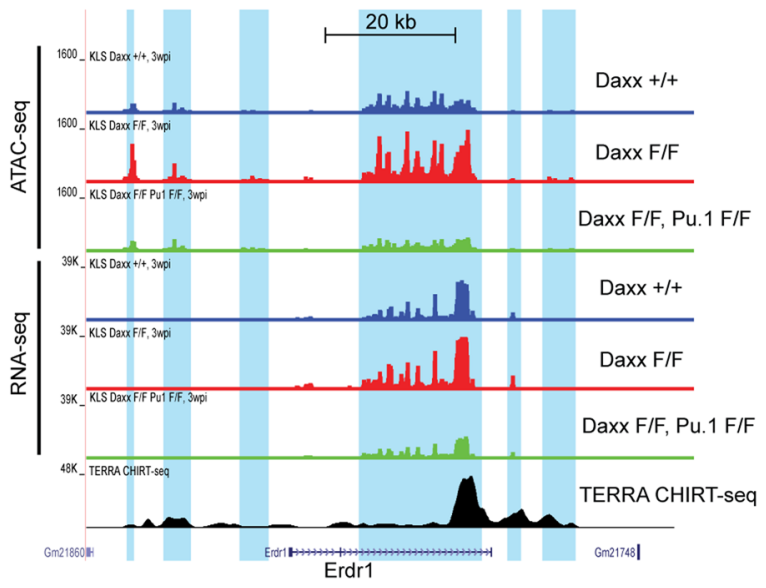

C

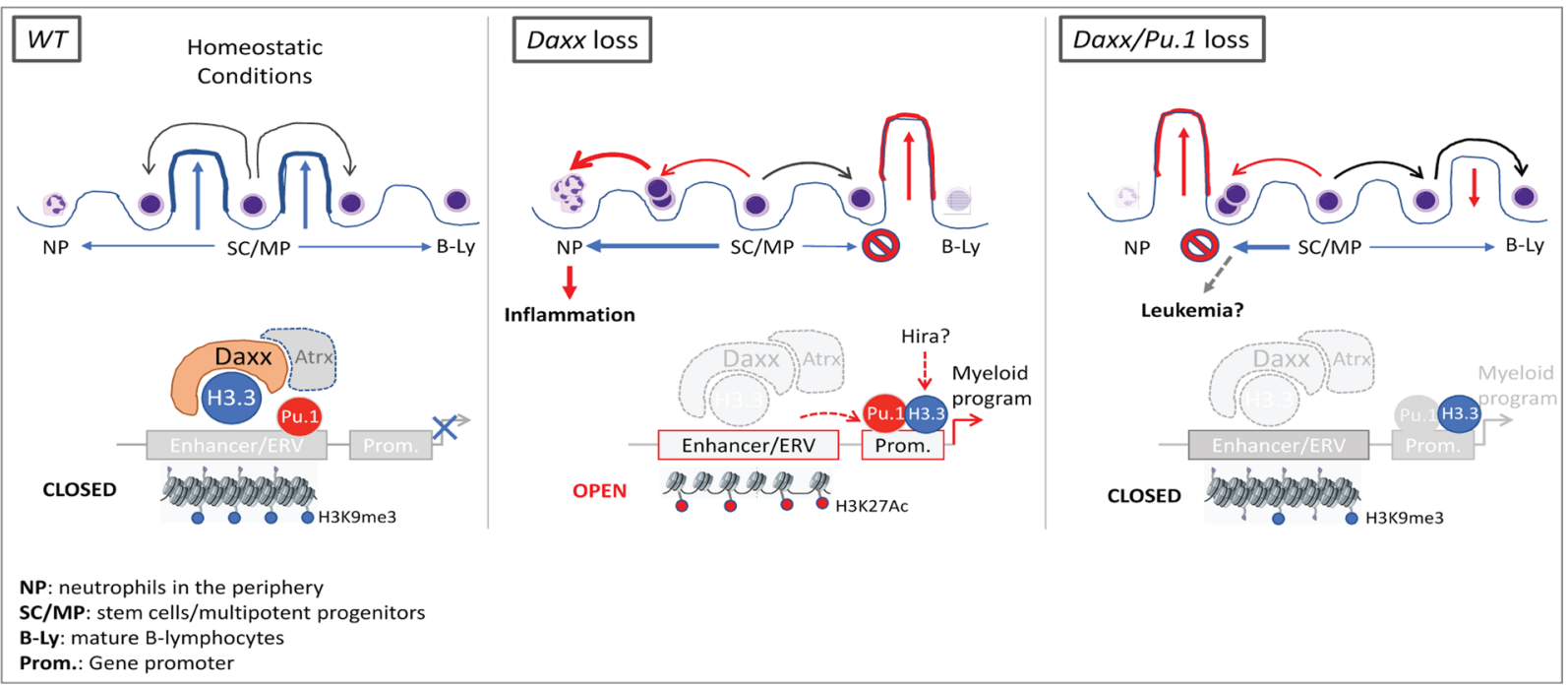

TERRA-BS on ChrX,Y

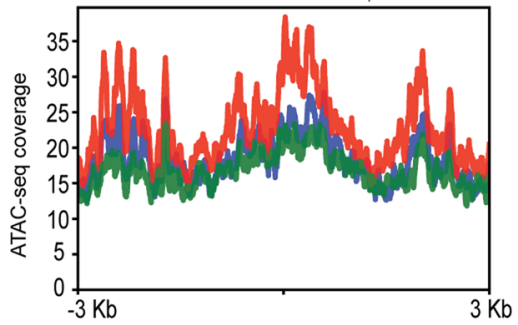

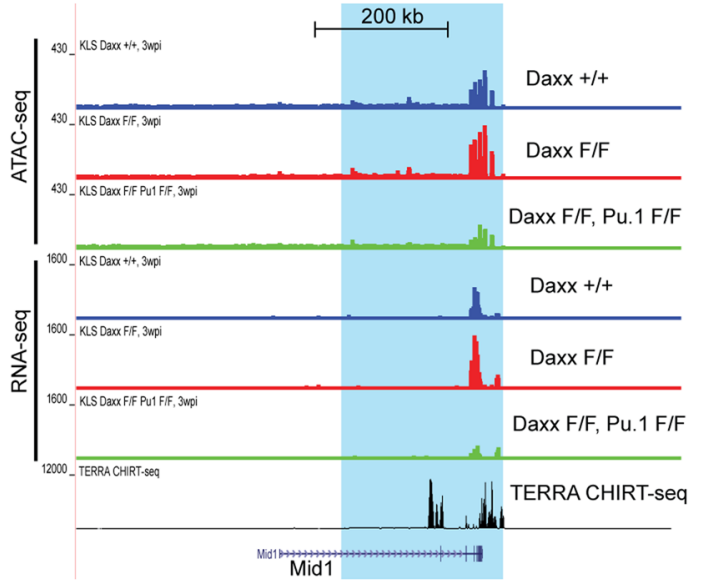

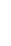




\section{Reporting Summary}

Nature Research wishes to improve the reproducibility of the work that we publish. This form provides structure for consistency and transparency in reporting. For further information on Nature Research policies, see Authors \& Referees and the Editorial Policy Checklist.

\section{Statistics}

For all statistical analyses, confirm that the following items are present in the figure legend, table legend, main text, or Methods section. $\mathrm{n} / \mathrm{a}$ Confirmed

$\bigotimes$ The exact sample size $(n)$ for each experimental group/condition, given as a discrete number and unit of measurement

\ A statement on whether measurements were taken from distinct samples or whether the same sample was measured repeatedly

$\varnothing$ The statistical test(s) used AND whether they are one- or two-sided

Only common tests should be described solely by name; describe more complex techniques in the Methods section.

Х $\square$ A description of all covariates tested

$\square$ A description of any assumptions or corrections, such as tests of normality and adjustment for multiple comparisons

$\square$ A full description of the statistical parameters including central tendency (e.g. means) or other basic estimates (e.g. regression coefficient)

AND variation (e.g. standard deviation) or associated estimates of uncertainty (e.g. confidence intervals)

For null hypothesis testing, the test statistic (e.g. $F, t, r$ ) with confidence intervals, effect sizes, degrees of freedom and $P$ value noted

Give $P$ values as exact values whenever suitable.

Х $\square$ For Bayesian analysis, information on the choice of priors and Markov chain Monte Carlo settings

Х $\square$ For hierarchical and complex designs, identification of the appropriate level for tests and full reporting of outcomes

$\bigotimes \square$ Estimates of effect sizes (e.g. Cohen's d, Pearson's $r$ ), indicating how they were calculated

Our web collection on statistics for biologists contains articles on many of the points above.

\section{Software and code}

\section{Policy information about availability of computer code}

Data collection

Primary processing of ATAC-seq data was performed with CASAVA (1.8.4), FastQC (0.11.5), Trimmomatic (0.36 with options -phred33, seedMismatches=2, palindromeClipThreshold=30, simpleClipThreshold=10 and option MINLEN:36), STAR (2.5.3 with option -alignIntronMax 1 and --alignMatesGapMax 1800), samtools (1.4.1), MarkDuplicates (option REMOVE_DUPLICATES=true) from Picard tools (2.9.2) and bedtools (2.25.0)

Primary processing of RNA-seq data was performed with CASAVA (1.8.4), FastQC (0.11.5), Cutadapt (1.13), STAR (2.5.3), samtools (1.4.1) and the PORT pipeline (https://github.com/itmat/Normalization).

CUT\&Tag was performed on HSPCs (Lineage-negative, c-Kit-positive) isolated by MACS. Primary processing was done with CASAVA (1.8.4), FastQC (0.11.5), bowtie2 (4.8.5), samtools (1.4.1) and bedtools (2.25.0).

A detailed description can be found in the Methods section and all tools and datasets used are listed in the Supplementary Information.

Data analysis

ATAC-seq data was analyzed using the ENCODE ATAC-seq analysis pipeline (0.3.4). Resulting peak files were annotated with the HOMER software package (4.9.1) as described in the Methods section. Genome Browser coverage plots and profile plots were created with deepTools (3.0.2) and Pathway analysis was performed with the Ingenuity Pathway analysis pipeline from Qiagen.

Statistical analysis was run in R (3.4.0) and GraphPad Prism (7.03), which were also used for plotting. Gene set enrichment analysis (GSEA) was performed with the GSEA program provided by the Broad Institute as described in the Methods section. Differential transposable element RNA expression was determined by applying the TEToolkit (2.0.2) as described in the Methods section. Differential expression analysis employed the R or Bioconductor packages GenomicFeatures (1.28.3), biomaRt (2.32.1), Rsamtools (1.28.0), GenomicAlignments (1.12.2) and DESeq2 (1.16.1). Genomic regions differentially enriched in H3.3, H3K27ac, H3K9me3, H3K27me3 or Pu.1 were called by using diffreps. Differential regions were annoted using the region_analysis package from diffreps.

FACS data was analyzed with FlowJo (10.6.1). 
Policy information about availability of data

All manuscripts must include a data availability statement. This statement should provide the following information, where applicable:

- Accession codes, unique identifiers, or web links for publicly available datasets

- A list of figures that have associated raw data

- A description of any restrictions on data availability

All sequencing data related to the manuscriptthat support the findings of this study can be found at Gene Expression Omnibus under the accession number GSE119309. An overview of the genomics studies run as part of this study can be found in Supplementary Table 11. Previously published sequencing data that were reanalyzed here are available under the accession codes GSE60101 and GSE79180 (SRR2062971 and SRR2062968). GTF and FASTA files used for Bioinformatics analysis (mm10, GENCODE release M14) can be downloaded from GENCODE (https://www.gencodegenes.org/mouse/release_M14.html). Source Data for Figs. 1-8 and Extended Data Figs. 1-8 are available online. All other data supporting the findings of this study are available from the corresponding author on reasonable request.

\section{Field-specific reporting}

Please select the one below that is the best fit for your research. If you are not sure, read the appropriate sections before making your selection.

$\bigotimes$ Life sciences $\quad \square$ Behavioural \& social sciences $\quad \square$ Ecological, evolutionary \& environmental sciences

For a reference copy of the document with all sections, see nature.com/documents/nr-reporting-summary-flat.pdf

\section{Life sciences study design}

All studies must disclose on these points even when the disclosure is negative.

Sample size Preliminary experiments were run to test for biological outcome. On the basis of the statistical anylsis of this data, the sample size required to achieve statistically significant results was calculated using Power Analysis. Sample sizes are noted in each case.

Data exclusions Data was partially excluded from the analysis of cytokine concentrations using Legendplex Mouse Inflammation Panel (Biolegend, 740446) and Mouse HSC Panel (Biolegend, 740677). The kits include a standard that is used for reference in the LEGENDplexTM Data Analysis Software. For several cytokines the analyzed concentrations of our samples were either not detectable or outside the analyzable range of the standard curve. In this case, single samples were excluded. Statistical analysis were run only if at least three biological replicates were available. The cytokines included in the kit that did not fall into the analyzable range of the standard curve are not shown as data in the manuscript.

Replication Experiments run on the same day always included wild-type and knock-out mice. For most experiments (except for ATAC-seq, RNA-seq and CUT\&Tag samples) included at least one replicate experiment run on a different date. The attempts of replication were successful and no experiment was excluded. All samples and mice were handled following the same protocols. Due to limitations in animals (numbers approved by authorities and COVID pandemic related restrictions for animal breeding) and complexity of required cell extraction, cell sorting and genomics library preparation, genomics-based experiments were run with biological replicates but without repetition at different dates.

Randomization Sample allocation was random and mice for experiments were randomly chosen based on availability of the right genotypes.

Blinding The investigators were not blinded to the mouse group allocation nor when assessing the outcome of experiments to ensure monitoring of mice for occurrence of skin lesions or signs of leukemia (in this case mice needed to be sacrificed). Blinding for analysis was not necessary as total cells are isolated from the different organs and cells are randomly assessed within the flow cytometer. To avoid bias during cell sorting (which are randomly assessed) the same sorting gates are used for all samples run on the same date.

\section{Reporting for specific materials, systems and methods}

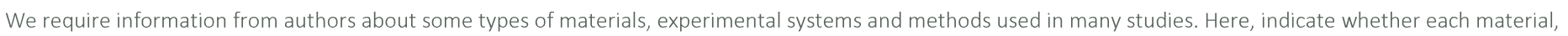

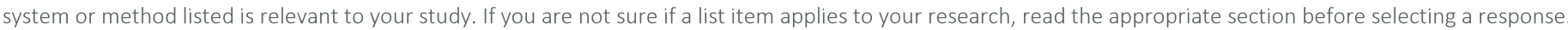

\begin{tabular}{l|l} 
Materials \& experimental systems \\
\hline n/a & Involved in the study \\
$\square$ & $\bigotimes$ Antibodies \\
$\square$ & $\square$ Eukaryotic cell lines \\
$\square$ & $\square$ Palaeontology \\
$\square$ & $\bigotimes$ Animals and other organisms \\
$\square$ & $\bigotimes$ Human research participants \\
$\triangle$ & $\square$ Clinical data
\end{tabular}

\begin{tabular}{l|l}
\multicolumn{2}{l}{ Methods } \\
\hline n/a & Involved in the study \\
$\square$ & $\bigotimes$ ChIP-seq \\
$\square$ & $\bigotimes$ Flow cytometry \\
$\square$ & $\square$ MRI-based neuroimaging
\end{tabular}


Antibodies used

Validation
Antibodies used are described in detail in Supplementary Table 12.

All antibodies used are commercially available antibodies and detailed validation information of each antibody can be found from the manufacturer, whose information can be found in Supplementary Table 12.

\section{Animals and other organisms}

Policy information about studies involving animals; ARRIVE guidelines recommended for reporting animal research

Laboratory animals

Wild animals

Field-collected samples

Ethics oversight
C57BL/6N Daxx F/F mice were crossed with C57BL/6J Csf1r-Cre mice (JAX 029206), C57BL/6J Mx1Cre mice (JAX 003556) or C57BL/6J Rosa26CreERT2 mice (JAX 008463). The C57BL/6N Hira conditional KO (HiraF/F) mice were crossed with C57BL/6J Mx1Cre mice (JAX 003556). The C57BL/6J Pu.1 conditional KO (Pu.1F/F; JAX 006922) were crossed with C57BL/6 DaxxF/F;Mx1Cre mice. For BM chimera experiments C57BL/6J CD45.1 mice were used (JAX 002014).

Activation of Cre in Daxx;RosaCreER mice was induced by administration of $80 \mathrm{mg} / \mathrm{kg}$ tamoxifen in corn oil (Sigma-Aldrich) via oral gavage on five consecutive days or by administration of $100 \mathrm{mg} / \mathrm{kg}$ tamoxifen in corn oil via i.p. injection on five consecutive days, two days break, followed by 2 more consecutive days. For induction of Cre activation Daxx KO, Hira KO and Daxx Pu.1 double KO Mx1Cre mice received intraperitoneal injection with $300 \mu \mathrm{g}$ polyinosinic-polycytidylic acid (pl:C, Sigma-Aldrich) three times every other day. Mice were usually treated between 5-11 weeks of age. Mice of both genders were used in this study. The $n$ value in Figure legends reflects the number of mice analyzed in each experiment.

\section{The study did not involve wild animals.}

The study did not involved field-collected samples.

All mouse experiments were performed in compliance with the UK and German Law for Welfare of Laboratory Animals and were approved by the Home Office in the UK (Project license 70-8240) as well as by the Landesamt fuer Natur, Umwelt und Verbraucherschuty (LANUV) of Nordrhein-Westfalen, Germany (Project license 84-02.04.2016.A486).

Note that full information on the approval of the study protocol must also be provided in the manuscript.

\section{Human research participants}

\section{Policy information about studies involving human research participants}

Population characteristics

Recruitment

Ethics oversight
We included H\&E stainings of skin from patients with Pyoderma gangraenosum in our study.

The text below is adapted from an email from Prof Wenzel that I have forwarded to the Editorial office.

The histological picture of the PG lesions has been taken from a skin sample that had been taken from a PG patient within the normal diagnostic procedure. This routine procedure includes the preparation of H\&E samples. Following German law is is fine to make pictures from these skin samples (-> it is not possible to recognize the patient and no additional investigations have been done with this sample), so a "patient consent" is not needed. This is also, as far as I know, in accordance with the Helsinki Ethical guidelines. However, the patient has given his informed consent to perform the skin biopsy within the diagnostic procedure.

Prof Wenzel has ethical Votum from the University of Bonn that allows to use skin material that had been taken for diagnostic proposes for research afterwards (BN090/04) in principle. but Prof Wenzel doesn't have a specific consent of this patient to make a picture of his H\&E slide, because it was not needed to do this.

Note that full information on the approval of the study protocol must also be provided in the manuscript.

\section{ChIP-seq}

\section{Data deposition}

$\bigotimes$ Confirm that both raw and final processed data have been deposited in a public database such as GEO.

\Confirm that you have deposited or provided access to graph files (e.g. BED files) for the called peaks.

Data access links

May remain private before publication.

Files in database submission

$$
\text { See Supplementary Table } 11
$$

Genome browser session

(e.g. UCSC)
Raw sequencing data was deposited in GEO under accession number GSE119309. 
https://genome.ucsc.edu/s/jentiger82/ATACseq_LTHSC_Daxx_ROSACreERmice;

https://genome.ucsc.edu/s/jentiger82/CUT\%26Tag_ATACseq_Daxx_Mx1Cre_3wpi;

https://genome.ucsc.edu/s/jentiger82/CUT\%26Tag_Daxx_Mx1Cre_8wpi;

https://genome.ucsc.edu/s/jentiger82/CUT\%26Tag_Daxx_Mx1Cre_24wpi

\section{Methodology}

Replicates

Sequencing depth

Antibodies

Peak calling parameters

Data quality

Software

\section{2-3 biological replicates}

about 10 million paired-end reads per sample

Antibodies against H3.3, Pu.1, H3K9me3, H3K27me3 and H3K27ac. Details can be found in Supplementary Table 12.

Online Methods

Online Methods

Online Methods

\section{Flow Cytometry}

Plots

Confirm that:

ХThe axis labels state the marker and fluorochrome used (e.g. CD4-FITC).

Х The axis scales are clearly visible. Include numbers along axes only for bottom left plot of group (a 'group' is an analysis of identical markers).

$\bigotimes$ All plots are contour plots with outliers or pseudocolor plots.

$\bigotimes$ A numerical value for number of cells or percentage (with statistics) is provided.

\section{Methodology}

Sample preparation

Instrument

Software

Cell population abundance

Gating strategy

Tick this box to confirm that a figure exemplifying the gating strategy is provided in the Supplementary Information.
Peripheral blood samples were collected from the tail vein of living mice. Bone marrow (BM) was isolated from femur and tibia of the hind legs and spleen cells from spleen of euthanized mice. Spleens were homogenized into single cells suspensions through a $70 \mu \mathrm{m}$ cell strainer. Unless red blood cells (RBC) were the cell population to be analyzed, RBC lysis was performed for $10 \mathrm{~min}$ in ammonium chloride and washed twice prior to antibody staining. Haematopoietic progenitor cells (HPC) were isolated from BM using the EasySep ${ }^{\text {TM }}$ Mouse Hematopoietic Progenitor Cell Isolation Kit (STEMCELL Technologies) according to manufacturer's instructions.

Flow cytometry analysis was performed on a BD LSR Fortessa, BD FACS Symphony, BD FACS Celesta, BD FACS Aria III or Beckman Coulter Gallios machine.

Cell sorting was performed on a BD FACS Aria III or BD FACS Aria Fusion machine using a $70 \mu \mathrm{m}$ or $100 \mu \mathrm{m}$ nozzle

Collection of data on BD FACS machines was performed by using BD FACSDIVA software. Collection of data on Beckman Coulter Gallios machines was performed by using Beckman Coulter KALUZA software. Data was analyzed using FlowJo (FlowJo, LLC) software.

For RNA sequencing CD45.2+ KLS (Lin-, CD45.2+, c-kit+, Sca1+) and GMP (Lin-, CD45.2+, c-kit+, Sca1-, CD16/32+, CD34+) populations were sorted from the bone marrow of transplanted mice. Haematopoietic stem cells (KLS) are of very low and GMP cells of low abundance in the bone marrow. Minimum 4700 cells were used for the RNA isolation. For ATAC seq sorting of CMP (Lin-, CD45.2+, c-kit+, Sca1-, CD16/32-, CD34+), GMP (Lin-, c-kit+, Sca1-, CD16/32+, CD34+) and long-term HSCs (Lin-, CD45.2+, ckit+,Sca1+, CD48-, CD150+) was performed. HSCs are of very low and GMP/CMP cells of low abundance in the bone marrow. Minimum 8000 cells were processed for ATACseq. To enrich frequency of haematopoietic stem cells in the sorting population, c-kit enrichment was performed prior to sorting of long-term HSCs.

To minimize the isolation of other cell populations during sorting, strict gating strategies were set based on FMO controls and unstained samples. Purity check of samples after sorting was performed by running small amounts of the sorted samples through the sorter.

The typical gating strategy was exclusion of cell debris on FSC/SSC plot, followed by single cell gating on FSC-H/FSC-A plot, followed by exclusion of dead cells using live/dead cells stains propidium iodide or Aqua Zombie fixable viability dye, followed by gating on the interesting cell populations based on the fluorochrome.

For setting positive and negative gates, unstained controls, single stained controls or FMO controls were used. 